\title{
THE EFFECT OF ACUPUNCTURE ON
}

\section{ALPHA-MOTONEURON EXCITABILITY}

Thesis submission to Auckland University of Technology for the Degree of

Master of Health Science, December, 2002

Alexander Kam Shing Chan

MB BS, PGDHSc (Western Acupuncture), FRNZCGP 


\section{TABLE OF CONTENTS}

The Effect of Acupuncture on Alpha-Motoneuron Excitability

Table of Contents

List of Figures

List of Tables

Certificate of Authorship

Acknowledgements

Abstract

\section{Chapter 1 Introduction}

1.1 Acupuncture and Pain Relief

1.2 Acupuncture and the Nervous System 3

1.2.1 Acupuncture Works through the Nervous System 3

1.2.2 Acupuncture Sensitive Receptors 4

1.2.3 Acupuncture, Deqi sensation and Afferent Fibres 5

$\begin{array}{ll}\text { 1.2.4 Acupuncture and Spinal Segmental Mechanisms } & 6\end{array}$

$\begin{array}{lll}\text { 1.2.5 Acupuncture and Heterosegmental effects } & 7\end{array}$

1.2.6 Acupuncture and the Cerebral Cortex and Subcortical Structures 9

1.2.7 Acupuncture and Diffuse Noxious Inhibitory Control 9

$\begin{array}{lll}1.3 & \text { Acupuncture and Humoral Mechanisms } & 10\end{array}$

$\begin{array}{lll}\text { 1.3.1 Acupuncture and Opioids } & 10\end{array}$

$\begin{array}{ll}\text { 1.3.2 Acupuncture and Other Neurotransmitters } & 11\end{array}$

1.4 Motor Control and Synaptic Inputs to $\alpha$-motoneurons 11

$\begin{array}{lll}1.5 & \text { Acupuncture and } \alpha \text {-Motoneuron Excitability } & 14\end{array}$

$\begin{array}{lll}1.6 & \text { The Hoffmann Reflex Methodology } & 17\end{array}$

1.7 Purpose of the Study 20

Chapter 2 Methodology 21

2.1 Objectives of the Study 21

2.2 Hypotheses 21

2.3 Methods 22

2.3.1 Introduction 22

2.3.2 Selection of test subjects 22

2.3.3 Stimulating and recording of H-reflex and M-waves 23

$\begin{array}{lll}\text { 2.3.3.1 Subject Position } & 23\end{array}$

$\begin{array}{lll}\text { 2.3.3.2 Skin Preparation } & 23\end{array}$

$\begin{array}{lll}\text { 2.3.3.3 Stimulating Electrodes Placement } & 24\end{array}$

2.3.3.4 Recording Electrodes and Earthing Electrode Placement 24

2.3.3.5 Equipment used for Stimulating and Recording 25

2.3.3.6 Stimulation and Recording of Reflex Responses 25

2.3.3.7 Acupuncture needles 26

2.3.4 H-reflex and M-wave Recordings 26

2.3.4.1 Criteria for Acceptance of an H-Reflex 26

2.3.4.2 Criteria for Determination of H-threshold $\left(\mathrm{H}_{\mathrm{th}}\right) \quad 27$ 
2.3.4.3 Criteria for Determination of maximum H-reflex $\left(\mathrm{H}_{\max }\right)$

2.3.4.4 Criteria for Determination of M-threshold $\left(\mathrm{M}_{\mathrm{th}}\right) \quad 27$

2.3.4.5 Criteria for Determination of M-maximum $\left(\mathrm{M}_{\max }\right) \quad 28$

2.3.5 Recruitment Curve Recording Protocol 28

$\begin{array}{ll}\text { 2.3.6 Experimental Design } & 29\end{array}$

2.3.7 Acupuncture points used 31

$\begin{array}{lll}\text { 2.3.7.1 GB34 (Yanglingquan) } & 31\end{array}$

$\begin{array}{lll}\text { 2.3.7.2 SP9 (Yinlingquan) } & 31\end{array}$

$\begin{array}{lll}2.3 .8 & \text { Acupuncture procedure } & 32\end{array}$

$2.4 \quad$ H-reflex and M-wave Recruitment Curves 32

2.4.1 Averaging of Response Amplitudes $\quad 32$

2.4.2 Data Normalisation $\quad 35$

2.4.3 Plotting of Recruitment Curves 36

2.4.3.1 The H-reflex Recruitment Curve 38

2.4.3.2 The M-wave Recruitment Curve 38

2.5 Linear Regression Analysis of H-reflex \& M-Wave Recruitment Curves 39

2.5.1 Calculation of H-slope $\left(\mathrm{H}_{\text {slp }}\right) \quad 40$

2.5.2 Calculation of M-slope $\left(\mathrm{M}_{\text {slp }}\right) \quad 44$

2.6 Analysis of M-wave Stability 46

$\begin{array}{lll}\text { 2.6.1 Intercept Method } & 47\end{array}$

2.6.2 Exceptions to Intercept Method 52

2.6.3 Reliability Test using Intraclass Comparison $\quad 54$

2.6.4 Analysis of Baseline Stability of the H-reflex 54

2.7 Assessment of Motoneuron Excitability by $\mathrm{H}_{\text {slp }}$ and $\mathrm{H}_{\text {slp }} / \mathrm{M}_{\text {slp }} \quad 54$

2.8 Assessment of Intensity of Acupuncture Sensation (deqi) 55

2.9 Statistical Analysis 55

Chapter 3 Results 57

3.1 Introduction 57

3.2 Effects of Short Duration Acupuncture $\quad 57$

3.2.1 M-slope stability 58

3.2.1.1 Analysis of M-slope Stability in Trials 1-4 (Preliminary) 58

3.2.1.2 Analysis of M-slope Stability in Trials 1-4 (Intercept Method) 58

3.2.2 Analysis of H-slopes Stability in Trials 1-2 61

$\begin{array}{ll}\text { 3.2.3 Changes in Motoneuron Excitability } & 61\end{array}$

$\begin{array}{lll}\text { 3.2.3.1 Changes in } \mathrm{H}_{\text {slp }} \text { after short acupuncture } & 61\end{array}$

3.2.3.2 Percentage Change in $\mathrm{H}_{\mathrm{slp}}$ after short duration acupuncture 64

3.2.3.3 Change in $\mathrm{H}_{\text {slp }} / \mathrm{M}_{\text {slp }}$ after short duration acupuncture 66

$\begin{array}{lll}\text { 3.2.3.4 Percentage Change in } \mathrm{H}_{\mathrm{slp}} / \mathrm{M}_{\mathrm{slp}} \text { after short duration acupuncture } & 68\end{array}$

$\begin{array}{lll}\text { 3.2.3.5 Conclusion } & 68\end{array}$

3.3 Effects of Short Duration Acupuncture (extended observation)

$\begin{array}{lll}\text { 3.3.1 M-slope stability } & 70\end{array}$

3.3.1.1 Analysis of M-slope Stability in Trials 1-5 (Preliminary) 70

3.3.1.2 Analysis of M-slope Stability in Trials 1-5 (Intercept Method) 70

$\begin{array}{lll}\text { 3.3.2 Analysis of H-slopes Stability in Trials 1-2 } & 71\end{array}$

$\begin{array}{lll}\text { 3.3.3 Changes in Motoneuron Excitability } & 73\end{array}$

3.3.3.1 Changes in $\mathrm{H}_{\text {slp }}$ after short duration acupuncture (extended observation) $\quad 73$ 
3.3.3.2 Percentage Change in $\mathrm{H}_{\text {slp }}$ after short duration acupuncture (extended observation)

3.3.3.3 Change in $\mathrm{H}_{\text {slp }} / \mathrm{M}_{\text {slp }}$ after short duration acupuncture (extended observation) $\quad 75$

3.3.3.4 Percentage Change in $\mathrm{H}_{\mathrm{slp}} / \mathrm{M}_{\mathrm{slp}}$ after short duration acupuncture (extended 79 observation)

3.3.3.5 Conclusion

3.4 Effects of Sustained Acupuncture $\quad 81$

3.4.1 M-slope stability 81

3.4.1.1 Analysis of M-slope Stability in Trials 1-4 (Preliminary) 81

3.4.1.2 Analysis of M-slope Stability in Trials 1-4 (Intercept Method) 81

3.4.2 Analysis of H-slopes Stability in Trials 1-2 84

3.4.3 Changes in Motoneuron Excitability 86

3.4.3.1 Changes in $\mathrm{H}_{\text {slp }}$ after sustained acupuncture $\quad 86$

3.4.3.2 Percentage Change in $\mathrm{H}_{\text {slp }}$ after sustained acupuncture $\quad 88$

3.4.3.3 Change in $\mathrm{H}_{\mathrm{slp}} / \mathrm{M}_{\mathrm{slp}}$ after sustained acupuncture $\quad 90$

3.4.3.4 Percentage Change in $\mathrm{H}_{\text {slp }} / \mathrm{M}_{\text {slp }}$ after sustained acupuncture 92

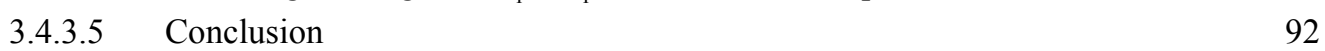

3.5 Effects of Sustained Acupuncture (extended observation) $\quad 94$

$\begin{array}{lll}\text { 3.5.1 M-slope stability } & 94\end{array}$

3.5.1.1 Analysis of M-slope Stability in Trials 1-5 (Preliminary) 94

3.5.1.2 Analysis of M-slope Stability in Trials 1-5 (Intercept Method) 94

3.5.2 Analysis of H-slopes Stability in Trials 1-2 98

3.5.3 Changes in Motoneuron Excitability 98

3.5.3.1 Changes in $\mathrm{H}_{\text {slp }}$ after sustained acupuncture (extended observation) 98

3.5.3.2 Percentage Change in $\mathrm{H}_{\text {slp }}$ after sustained acupuncture (extended 100 observation)

3.5.3.3 Change in $\mathrm{H}_{\text {spp }} / \mathrm{M}_{\text {slp }}$ after sustained acupuncture (extended observation) 102

3.5.3.4 Percentage Change in $\mathrm{H}_{\mathrm{slp}} / \mathrm{M}_{\mathrm{slp}}$ after sustained acupuncture (extended 104 observation)

$\begin{array}{lll}\text { 3.5.4 Conclusion } & 106\end{array}$

3.6 Comparison of effects of short duration and sustained acupuncture on 107 motoneuron excitability

3.7 Effect of intensity of acupuncture sensation on $\alpha$-motoneuron excitability $\quad 111$

3.8 Summary of Results 113

$\begin{array}{lll}\text { Chapter } 4 \text { Discussion } & 115\end{array}$

4.1 Major findings 115

4.2 Acupuncture and $\alpha$-Motoneuron Excitability 116

$\begin{array}{lll}\text { 4.2.1 Effects of sites of acupuncture and H-reflex recordings } & 116\end{array}$

$\begin{array}{ll}\text { 4.2.2 Effect of timing the H-reflex recordings } & 117\end{array}$

$\begin{array}{ll}\text { 4.2.3 Effect of amount of afferent input from acupuncture } & 117\end{array}$

$\begin{array}{lll}\text { 4.2.4 Effect of manual acupuncture versus electroacupuncture } & 118\end{array}$

$\begin{array}{lll}\text { 4.2.5 Effect of different H-reflex methodology } & 119\end{array}$

$\begin{array}{lll}\text { 4.2.6 Effect of specificity of acupuncture points } & 120\end{array}$

$\begin{array}{lll}\text { 4.2.7 Difference between healthy subjects and stroke patients } & 121\end{array}$

$\begin{array}{lll}4.2 .8 & \text { Variation of individual responses after acupuncture } & 122\end{array}$

4.2.9 Change in $\alpha$-motoneuron excitability did not arise from inactivity 123

$\begin{array}{lll}\text { 4.2.10 Effect of short duration acupuncture may not be passive } & 123\end{array}$ 
4.3 Comparison of effect of acupuncture and other interventions on $\alpha$ - 124 motoneuron excitability

4.4 Limitations of the Study 125

$\begin{array}{ll}\text { 4.4.1 Limitations related to changes in peripheral conditions } & 125\end{array}$

$\begin{array}{ll}\text { 4.4.2 Procedures for screening peripheral stability } & 125\end{array}$

$\begin{array}{lll}\text { 4.4.3 Compensation for minor changes in peripheral conditions } & 126\end{array}$

$\begin{array}{ll}\text { 4.4.4 Limitations of cross-over design } & 127\end{array}$

4.5 Intensity of Acupuncture Sensation and $\alpha$-Motoneuron Excitability 127

4.6 Possible Mechanisms of Acupuncture Effect on $\alpha$-motoneuron Excitability 129

$\begin{array}{ll}\text { 4.6.1 The effect of acupuncture on the mental state of the subjects } & 129\end{array}$

$\begin{array}{ll}\text { 4.6.2 The effect of acupuncture on } \alpha \text {-motoneurons excitability } & 130\end{array}$

$\begin{array}{lll}4.7 & \text { Clinical Significance } & 132\end{array}$

4.8 Future Work 133

$\begin{array}{lll}\text { APPENDIX I } & \text { Participant Information Sheet }\end{array}$

APPENDIX II Consent to Participation in Research 138

$\begin{array}{lll}\text { APPENDIX III } & 139\end{array}$

$\begin{array}{lll}\text { APPENDIX IV Subject data recording sheet } & 140\end{array}$

APPENDIX V Table of M-intercepts confidence intervals in short duration 142 acupuncture study

APPENDIX VI Table of M-intercepts confidence intervals in sustained 143

$\begin{array}{lll}\text { APPENDIX VII } & \text { Discarded experiments }\end{array}$

References $\quad 145$ 


\section{LIST OF FIGURES}

$\begin{array}{llr}\text { Fig. 1.1 The H-reflex stimulation and recording system } & 19\end{array}$

Fig. 2.1A H-Reflex and M-Wave Recruitment Curves constructed from raw data 34

Fig. 2.1B H-reflex and M-wave Recruitment Curves constructed from averaged amplitudes 34

Fig. 2.2 Normalised H-Reflex and M-Wave Recruitment Curves 37

Fig. 2.3 Linear regression analysis of the ascending slope of the H-reflex recruitment curve 43

Fig. 2.4 Linear regression analysis of the ascending slope of an M-wave recruitment curve 45

Fig. 2.5 Examination of M-wave stability using the Intercept method 51

Fig. 2.6 Overlapped M-wave recruitment curves with non-overlapping $y$-intercept 53

Fig. 3.1A Changes in $M_{\text {slp }}$ in Trials 1-4 in short duration acupuncture ( $\left.n=20\right) \quad 60$

Fig. 3.1B Changes in $M_{\text {slp }}$ in Trials 1-4 in short duration acupuncture ( $\left.n=16\right) \quad 60$

Fig. 3.2 Changes in $\mathrm{H}_{\text {slp }}$ (baseline, Trials 3-4) in short duration acupuncture $(\mathrm{n}=16) \quad 63$

Fig. 3.3 Percentage changes in $\mathrm{H}_{\text {slp }}$ in Trials 3-4 after short duration acupuncture $(\mathrm{n}=16) \quad 65$

Fig. 3.4 Changes in $\mathrm{H}_{\text {slp }} / \mathrm{M}_{\text {slp }}$ (baseline, Trials 3-4) in short duration acupuncture $(\mathrm{n}=16) \quad 67$

Fig. 3.5 Percentage changes in $\mathrm{H}_{\text {slp }} / \mathrm{M}_{\text {slp }}$ in Trials 3-4 after short duration acupuncture 69

Fig. 3.6A Changes in $\mathrm{M}_{\text {slp }}$ in Trials 1-5 in short duration acupuncture (extended observation; 72

Fig. 3.6B $\quad \begin{array}{ll}\mathrm{n}=10) \\ \mathrm{n}=8 \text { ) }\end{array}$

Fig. 3.7 Changes in $\mathrm{H}_{\text {slp }}$ (baseline, Trials 3-5) in short duration acupuncture (extended 74

Fig. 3.8 Percentage changes in $\mathrm{H}_{\text {slp }}$ in Trials 3-5 after short duration acupuncture (extended 76

Fig. $3.9 \quad$ Changes in $\mathrm{H}_{\text {slp }} / \mathrm{M}_{\text {slp }}$ (baseline, Trials 3-5) in short duration acupuncture (extended 78

Fig. 3.10 Percentage changes in $\mathrm{H}_{\text {slp }} / \mathrm{M}_{\text {slp }}$ in Trials 3-5 after short duration acupuncture 80

Fig. 3.11A Changes in $M_{\text {slp }}$ in Trials 1-4 in sustained acupuncture $(n=20)$

Fig. 3.11B Changes in $\mathrm{M}_{\text {slp }}$ in Trials 1-4 in sustained acupuncture $(\mathrm{n}=16)$

Fig. 3.12A Changes in $\mathrm{H}_{\text {slp }}$ in Trials 1-2 in sustained acupuncture $(\mathrm{n}=16)$

Fig. 3.12B Changes in $\mathrm{H}_{\text {slp }}$ in Trials 1-2 in sustained acupuncture $(\mathrm{n}=15)$

Fig. 3.13 Changes in $\mathrm{H}_{\text {slp }}$ (baseline, Trials 3-4) in sustained acupuncture $(\mathrm{n}=15) \quad 87$

Fig. 3.14 Percentage changes in $\mathrm{H}_{\text {slp }}$ in Trials 3-4 after sustained acupuncture $(\mathrm{n}=15) \quad 89$

Fig. 3.15 Changes in $\mathrm{H}_{\text {slp }} / \mathrm{M}_{\text {slp }}$ (baseline, Trials 3-4) in sustained acupuncture ( $\left.\mathrm{n}=15\right) \quad 91$

Fig. 3.16 Percentage changes in $\mathrm{H}_{\text {slp }} / \mathrm{M}_{\text {slp }}$ in Trials 3-4 after sustained acupuncture (n=15) 93

Fig. 3.17A Changes in M-slopes in Trials 1-5 in sustained acupuncture (extended 96

Fig. 3.17B Changes in M-slopes in Trials 1-5 in sustained acupuncture (extended 96

Fig.3.17C Changes in M-slopes in Trials 1-5 in sustained acupuncture (extended 97

Fig. 3.18 Changes in $\mathrm{H}_{\text {slp }}$ (baseline, Trials 3-5) in sustained acupuncture (extended 99

Fig. 3.19 Percentage changes in $\mathrm{H}_{\text {slp }}$ in Trials 3-5 after sustained acupuncture $(\mathrm{n}=8) \quad 101$ 
Fig. 3.20 Changes in $\mathrm{H}_{\text {slp }} / \mathrm{M}_{\text {slp }}$ (baseline, Trials 3-5) in sustained acupuncture (extended observation; $\mathrm{n}=8$ )

Fig. 3.21 Percentage changes in $\mathrm{H}_{\text {slp }} / \mathrm{M}_{\text {slp }}$ in Trials 3-5 after sustained acupuncture (extended 105 observation; $\mathrm{n}=8$ )

Fig. 3.22 Relative changes in the means of $\mathrm{H}_{\text {slp }}$ before and after short duration and sustained acupuncture $(\mathrm{n}=13)$

Fig. 3.23 Relative percentage changes in $\mathrm{H}_{\text {slp }}$ in Trials 3-4 after short duration and sustained acupuncture $(\mathrm{n}=13)$

Fig. 3.24 Relative changes in $\mathrm{H}_{\text {slp }} / \mathrm{M}_{\text {slp }}$ before and after short duration and sustained

Fig. 3.25 Relative percentage changes in $\mathrm{H}_{\mathbf{s l p}} / \mathrm{M}_{\text {slp }}$ in Trials 3-4 after short duration and sustained acupuncture $(n=13)$ 


\section{LIST OF TABLES}

Table $2.1 \quad$ The experimental protocol used in the study

Table 2.2 Normalised Averaged M-wave Amplitudes versus Normalised Stimulation

Table 2.3 Linear Regression Analysis of averaged M-wave amplitudes in Table 2.2 using SPSS

Table 2.4 Normalised Pre-averaged M-wave Amplitudes verus Normalised Stimulation Intensities

Table 2.5 Linear Regression Analysis of pre-averaged M-wave amplitudes in Table 2.4 using SPSS

Table 3.1 Descriptive statistics of $\mathrm{H}_{\text {slp }}$ (baseline, Trials 3 and 4) in short duration acupuncture

Table 3.2 Descriptive statistics of percentage changes in $H_{\text {slp }}$ in Trials 3 and 4 after short duration acupuncture $(\mathrm{n}=16)$

Table 3.3 Descriptive statistics of $\mathrm{H}_{\text {slp }} / \mathrm{M}_{\text {slp }}$ (baseline, Trials 3 and 4 ) in short duration acupuncture $(\mathrm{n}=16)$

Table 3.4 Descriptive statistics of percentage changes in $H_{\text {slp }} / M_{\text {slp }}$ in Trials 3 and 4 after short duration acupuncture $(n=16)$

Table 3.5 Descriptive statistics of $\mathrm{H}_{\text {slp }}$ (baseline, Trials 3-5) in short duration acupuncture (extended observation; $n=8$ ).

Table 3.6 Descriptive statistics of percentage changes in $\mathrm{H}_{\text {slp }}$ in Trials 3-5 after short duration acupuncture (extended observation; $n=8$ )

Table 3.7 Descriptive statistics of $\mathrm{H}_{\text {slp }} / \mathrm{M}_{\text {slp }}$ (baseline, Trials 3-5) in short acupuncture (extended observation; $\mathrm{n}=8$ )

Table 3.8 Descriptive statistics of percentage changes in $\mathrm{H}_{\text {slp }} / \mathrm{M}_{\text {slp }}$ in Trials 3-5 after short duration acupuncture (extended observation; $n=8$ ).

Table 3.9 Descriptive statistics of $\mathrm{H}_{\text {slp }}$ (baseline, Trials 3-4) in sustained acupuncture $(\mathrm{n}=15)$

Table 3.10 Descriptive statistics of percentage changes in $\mathrm{H}_{\text {slp }}$ in Trials 3-4 after sustained acupuncture $(\mathrm{n}=15)$

Table 3.11 Descriptive statistics of $\mathrm{H}_{\mathbf{s l p}} / \mathrm{M}_{\text {slp }}$ (baseline, Trials 3-4) in sustained acupuncture $(\mathrm{n}=15)$

Table 3.12 Descriptive statistics of percentage changes in $\mathrm{H}_{\text {slp }} / \mathrm{M}_{\text {slp }}$ in Trials 3-4 after sustained acupuncture $(\mathrm{n}=15)$

Table 3.13 Descriptive statistics of $\mathrm{H}_{\text {slp }}$ (baseline, Trials 3-5) in sustained acupuncture (extended observation; $\mathrm{n}=8$ )

Table 3.14 Descriptive statistics of percentage changes in $\mathrm{H}_{\text {slp }}$ in Trials 3-5 after sustained acupuncture $(\mathrm{n}=8)$

Table 3.15 Descriptive statistics of $\mathrm{H}_{\text {slp }} / \mathrm{M}_{\text {slp }}$ (baseline, Trials 3-5) in sustained acupuncture (extended observation; $n=8$ )

Table 3.16 Descriptive statistics of percentage changes in $\mathrm{H}_{\text {slp }} / \mathrm{M}_{\text {slp }}$ in Trials 3-5 after sustained acupuncture (extended observation; $n=8$ )

Table 3.17 Results of repeated measures ANOVA comparing different parameters of $\alpha$-motoneuron excitability in short duration and sustained acupuncture

Table 3.18 Correlation between Intensity of Acupuncture Sensation and Changes in Hreflex (Short Duration Acupuncture)

Table 3.19 Correlation between Intensity of Acupuncture Sensation and Changes in Hreflex (Sustained Acupuncture)

Table $3.20 \quad$ Summary of results in short duration and sustained acupuncture studies Effects of duration of acupuncture and time interval after acupuncture on

Table 3.21 motoneuron excitability $(\mathrm{n}=13)$ 


\section{CERTIFICATE OF AUTHORSHIP}

I hereby declare that this submission is my own work and that, to the best of my knowledge and belief, it contains no material previously published or written by another person nor material which to a substantial extent has been accepted for the award of any other degree or diploma of a university or other institution of higher learning, except where due acknowledgement is made in the acknowledgements. 


\section{ACKNOWLEDGEMENTS}

It has been a very steep learning curve in the last 18 months working on this thesis. The whole process was very demanding but definitely worthwhile because the foundation of a pathway to acquire further new knowledge has now been laid. For this, I would like to formally acknowledge the expert guidance and support of my supervisors, Dr. Andrea Vujnovich and Mrs. Lynley Roberts. In addition, I would like to thank them for their tolerance, encouragement, understanding, constructive criticism, easy accessibility and generous amount of time they gave me during this period.

I would like to thank Mr. Maynard Williams for his expert advice regarding the statistical analysis, Ms Jill Kerr for her tips on various aspects of the project, the subjects who took part in the experiments, the staff from the Library of the Auckland University of Technology, and the staff from my own general practice in the Windsor Medical Centre in Mairangi Bay.

I would like to acknowledge the financial support from the Charitable Trust of the Faculty of the Royal New Zealand College of General Practitioners for Research.

I would also like to thank the encouragement from my children, Donald and Velda, who always have confidence in their dad completing the thesis on time, the unending inspiration from our "god-grandson" Jonathan (alias soft-toy), and most of all, my wife Helen who led me walking this path. Last but not least, I wish to express my sincere thanks to the Universal Creator who has given me this opportunity to live and learn from all these loving people around me. 


\begin{abstract}
The analgesic effect of acupuncture is well known. Areas in the brain and higher centres that are activated by acupuncture have been mapped out. Some of these areas are also implicated in the modulation of motor function. In addition to pain relief, acupuncture has been found to increase range of movement in patients with increased muscle tone. There is, however, scant knowledge of the mechanisms underlying this therapeutic effect.
\end{abstract}

The purpose of this study was to investigate the effect of manual acupuncture on excitability of the $\alpha$-motoneuron pool. The effect of duration of acupuncture and significance of the intensity of acupuncture sensation (deqi) were also examined. The study was approved by the Auckland University of Technology Ethics Committee.

Twenty (20) asymptomatic subjects participated in the study after giving informed consent. The Soleus H-reflex response to acupuncture stimulation at GB 34 and SP 9 in the same leg was used as a tool for indirectly assessing the $\alpha$-motoneuron pool excitability. H-reflexes were evoked and recorded according to established criteria.

Each subject received short duration acupuncture (5 minutes) and sustained acupuncture (20 minutes) at least 2 weeks apart, but the sequence was randomised. Two control Hreflex recruitment curves (Trials 1 and 2) were recorded before acupuncture. H-reflex recruitment curves were then taken on withdrawal of needles (Trial 3) and again 15 minutes later (Trial 4). A proportion of subjects had further H-reflex recordings 30 minutes after acupuncture for delayed effects (Trial 5). Intensity of acupuncture 
sensation (deqi) was recorded by each subject using a $100 \mathrm{~mm}$. horizontal visual analogue scale.

Changes in the H-reflex were analysed using the parameters of $\mathrm{H}_{\text {slp }}$ and $\mathrm{H}_{\text {slp }} / \mathrm{M}_{\text {slp }}$ after establishing peripheral stability by examining $\mathrm{M}_{\text {slp }}$ consistency in the same experiment. $\mathrm{H}_{\text {slp }}$ and $\mathrm{M}_{\mathrm{slp}}$ were calculated by linear regression analysis of the ascending slope of the respective recruitment curves. A significant suppression of $\alpha$-motoneuron excitability was found 15 minutes after sustained acupuncture. The effect was still present at 30 minutes on further recording. There was no significant change in $\alpha$-motoneuron excitability after short acupuncture at any stage. However, repeated measures analysis of variance (ANOVA) revealed the difference between short duration and sustained acupuncture was not statistically significant.

Correlation between the intensity of perceived acupuncture sensation (deqi) and suppression of $\mathrm{H}_{\mathrm{slp}}$ and $\mathrm{H}_{\mathrm{slp}} / \mathrm{M}_{\mathrm{slp}}$ was found to be statistically insignificant in both short duration and sustained acupuncture studies.

In conclusion, sustained acupuncture had stronger influence on the central nervous system than short duration acupuncture. It evoked a delayed inhibition of the $\alpha$ motoneuron pool excitability which was still present 30 minutes after the intervention. The time course of action suggested a neurohumoral mechanism. Intensity of acupuncture sensation (deqi) did not appear to have a significant correlation to the effect of acupuncture on $\alpha$-motoneuron excitability. 


\section{Chapter 1}

\section{Introduction}

Acupuncture is one of the ancient Chinese methods for health maintenance and disease management. The practice of acupuncture has historically been based on the theoretical concepts of Traditional Chinese Medicine and the experience of the practitioners. The report by Reston (Reston, 1971) which outlined the remarkable analgesic effects of acupuncture in the early 70's generated huge amounts of interest in the West regarding its clinical usage and scientific basis. The results of numerous studies were published in peer-reviewed medical journals over the next 30 years (Han, 1997; Hsieh et al., 2001; Huang, Wang, Han, \& Wan, 2002; Murray, 1995; Ulett, Han, \& Han, 1998). By 1997, the National Institute of Health (NIH) Office of Alternative Medicine Consensus Panel (Acupuncture) concluded that acupuncture is effective in reducing nausea and vomiting associated with postoperative anaesthesia and chemotherapy, and in reducing postoperative dental pain. The same Consensus Panel also determined acupuncture could be a useful adjunct in the management of other conditions such as addiction, stroke rehabilitation, headache, menstrual cramps, tennis elbow, fibromyalgia, myofascial pain, osteoarthritis, low back pain, carpal tunnel syndrome, and asthma.

Research into the scientific basis of acupuncture has been focussed mainly on its analgesic effects and neurohumoral connections (Han et al., 1980; He \& Dong, 1983; Lee \& Beitz, 1993; Pan, Castro-Lopes, \& Coimbra, 1996; Pomeranz, 2001). There has been little research into the effect of acupuncture on motor control. However, it is possible that some of the pain modulating pathways activated by acupuncture could also influence the excitability of $\alpha$-motoneurons. This hypothesis is supported by the discovery of multitudinous synaptic inputs to motoneurons from both spinal and 
supraspinal sources (Alstermark, Kummel, Pinter, \& Tantisira, 1990; Holstege \& Kuypers, 1987; Ornung, Ottersen, Cullheim, \& Ulfhake, 1998; Rekling, Funk, Bayliss, Dong, \& Feldman, 2000). A review of the literature surrounding the neurophysiology of acupuncture analgesia and motor control shows a possible link between the two.

\subsection{Acupuncture and Pain Relief}

Acupuncture produces analgesia in both animals and humans. Several techniques have been developed to assess changes in pain threshold in rats. These include the latency of tail flick, squeak and limb withdrawal to radiant heat, and of the jaw opening reflex to noxious stimulus (Dai, Zhu, Li, Huang, \& Xu, 1992; Kawakita \& Funakoshi, 1982; Lou, Sun, Liu, \& Tong, 1992; Pomeranz \& Paley, 1979). An increase in latency of the response after intervention would indicate an increase in pain threshold as a result of the intervention. Acupuncture has been shown to increase the latencies of all these reflexes in rats (Dai et al., 1992; Kawakita \& Funakoshi, 1982; Lou et al., 1992; Pomeranz \& Paley, 1979). In addition, in the rat model, discharges of wide dynamic range (WDR) neurons in the dorsal horn of the spinal cord elicited by stimulation of $\mathrm{C}$ fibres, which conduct nociceptive impulses, was shown to be inhibited by manual acupuncture (Hashimoto, Akita, \& Aikawa, 1993).

Pain threshold in healthy human subjects was also found to be raised by acupuncture (Chiang, Chang, Chu, \& Yang, 1973; Lundeberg, Eriksson, Lundeberg, \& Thomas, 1989). Interestingly, the rise in pain threshold appeared to be more prominent in acupuncture points tested along the acupuncture meridian than in non-acupuncture points further away from the meridian (Farber, Tachibana, \& Campiglia, 1997). Acupuncture also increased pain threshold in patients suffering from chronic tension 
headaches (Karst, Rollnik, Fink, Reinhard, \& Piepenbrock, 2000) and the subjective changes in pain threshold lasted several hours (Leong \& Chernow, 1988). Clinically, acupuncture has been found to be effective in reducing the need for epidural analgesia during labour (Ramnero, Hanson, \& Kihlgren, 2002), provided a longer pain free period after dental surgery (Lao, Bergman, Langenberg, Wong, \& Berman, 1995), and reduced the need for intraoperative and postoperative analgesia (Sim, Xu, Pua, Zhang, \& Lee, 2002). A recent study demonstrated that acupuncture was superior to sham acupuncture in reducing pain and in increasing the range of movement in chronic neck pain patients (Irnich et al., 2002). A meta-analysis of trials also found acupuncture to be effective in the treatment of low back pain (Ernst, White, \& Wider, 2002). Thus, there is evidence that acupuncture has an analgesic effect both in animal models and humans. Clinically, the analgesic effect of acupuncture is present not only during the intervention itself, but also extends into the post-acupuncture period.

\subsection{Acupuncture and the Nervous System}

\subsubsection{Acupuncture Works through the Nervous System}

The evidence that the effects of acupuncture are mediated by the nervous system was provided by Chiang et al (1973). Pain thresholds in various parts of the body were found to be significantly raised after acupuncture at LI 4 (Hoku) and LI 10 (Shousanli) in the left upper limb. A tourniquet in the left upper arm to occlude the circulation did not affect the rise in pain thresholds after acupuncture. This indicated that the effects were not mediated by circulation of humoral substances from the needling sites. In addition, after the transmission of nerve impulses from the acupuncture points was blocked by local injection of procaine, acupuncture did not result in elevation of the pain thresholds, as opposed to the control experiments in which saline was injected. Chiang 
et al (1973), therefore, concluded that unimpaired function of the peripheral nerve was indispensable for the mediation of acupuncture analgesia. In an animal model, a group of mice was found to have compound nerve potentials in the brachial plexus during electroacupuncture to the upper limb. This phenomenon was associated with a raised pain threshold demonstrated as a delayed squeak response to heat. Neither of these effects was present in another group which had xylocaine injected into the site of electroacupuncture (Pomeranz \& Paley, 1979). This indicated that the analgesic effect from electroacupuncture was most likely related to nerve impulses being transmitted to the central nervous system from the acupuncture site.

\subsubsection{Acupuncture Sensitive Receptors}

Acupuncture generally involves inserting a needle through the skin, and depending on the site, into underlying deeper structures such as muscles. When the needle goes through the skin, it gives rise to a pricking sensation. The insertion may be associated with a red flare in the skin at the penetration site and a warm sensation which is thought to result from the axon reflex brought about by stimulation of free nerve endings associated with A $\delta$ and $\mathrm{C}$ fibres (Bowsher, 1998). Depending on the sites of the acupuncture points, further insertion of the needle will evoke discharges from receptor units such as high-threshold ergoreceptors (Andersson \& Lundeberg, 1995), touch or pressure receptors, muscle spindles, and Golgi tendon receptors units (Wang \& Liu, 1989). Wang et al. (1989) found receptor units with different sensitivity and sensation characteristics within the same acupuncture point area. By using microneurography of individual receptors Kawakita et al. (1996) demonstrated that some receptors were polymodal and capable of responding to different stimuli. Therefore, acupuncture points are well equipped to respond to various forms of inputs such as heat, pressure, mechanical and electrical stimulation which are used in clinical acupuncture practice. 


\subsubsection{Acupuncture, Deqi sensation and Afferent Fibres}

With further insertion of the needle into the muscle, a peculiar sensation is elicited. This is the acupuncture sensation (deqi) and is variously described as aching, pulling, heavy, dull, numb, full, electric and throbbing (Hsieh et al., 2001; Park, Park, \& Lee, 2002).

The presence of acupuncture sensation (deqi) is considered to be a pre-requisite for effective acupuncture by traditional acupuncturists (Cheng, 1987; Helms, 1995). However, it has been reported that a difference in the perception of needling sensation occurred among different individuals and at different points on the same individual (Li, Jiang, \& Chen, 1983). Despite this, Li et al (1983) reported a close correlation of needling sensation with needling effect. Their experiment showed strong needling sensation was accompanied by marked elevation of individual pain threshold and depression of somatosensory evoked potentials recorded from the vertex of the head ( $\mathrm{Li}$ et al., 1983).

Using direct microelectrode recordings from single fibres, Wang et al (1985) demonstrated that during acupuncture Group II (A $\beta$ ) muscle afferents produced numbness, Group III (A $\delta$ ) afferents produced distension and heaviness, and Group IV (C) afferents produced soreness when the acupuncture sensation (deqi) was present (Wang et al., 1985). Group III (A $\delta$ ) fibres are also known to convey pinprick and Group IV (C) fibres to convey aching pain sensations (Guyton \& Hall, 2000). Acupuncture induced sensation (deqi), therefore, occurs as a result of one or a combinations of these afferent fibres being stimulated.

From his experiments, Han (1997) pointed out that while increased intensity of stimulation to involve more Group IV (C) fibres during electroacupuncture would 
increase the potency of analgesia, the pain and stress elicited by these noxious stimuli would prevent it from clinical use. Pomeranz and Paley (1979) found that Group II (A $\beta$ ) afferents were sufficient to produce analgesia with electroacupuncture. It is agreed by some investigators that Group II (A $\beta)$ and Group III (A $\delta)$ afferent units are responsible for experience of acupuncture sensation and must be stimulated in order to produce the electroacupuncture effect (Bowsher, 1998; Han, 1997; Pomeranz, 2001).

On the other hand, Kawakita et al (Kawakita \& Gotoh, 1996), using microneurography, found sustained discharges from $\mathrm{A} \delta$ deep receptor and $\mathrm{C}$ fibre receptor when manual acupuncture stimulation was applied to their receptive fields. Okada et al (1996) also found that after blocking Group III (A $\delta$ ) and IV (C) fibres of the sciatic nerves by using capsaicin, manual acupuncture of the hind paw no longer resulted in causing suppression of jaw-opening reflex in rats. Therefore, it is very likely that Group III (A $\delta)$ and Group IV (C) afferent fibres, mediated by polymodal receptors, are activated by manual acupuncture as distinct from Group II (A $\beta)$ and Group III (A $\delta)$ afferent fibres by electroacupuncture.

\subsubsection{Acupuncture and Spinal Segmental Mechanisms}

At the spinal cord level, collaterals of A $\delta$ fibres activated by acupuncture stimulation either synapse directly or indirectly via anterolateral tract cells with inhibitory endorphinergic interneurons within the same segment. These in turn release enkephalin or dynorphin to presynaptically inhibit the nociceptive input from the $\mathrm{C}$ afferent fibres and postsynaptically inhibit the spinothalamic tract cells which convey the nociceptive input to higher centres (Bowsher, 1998; Pomeranz, 2001). Thus, acupuncture may block pain transmission at the spinal cord level. 
Chung et al. (1984) investigated the segmental effect of acupuncture by measuring the influence of electrical peripheral nerve stimulation on the activities of the spinothalamic tract cells in spinalised monkeys. It was found that activities in the spinothalamic tract cells elicited by noxious heat and electrical stimulation of the sural nerve sufficient to activate $\mathrm{C}$ fibres were greatly inhibited by repetitive conditioning stimuli applied either to the common peroneal or tibial nerve. The inhibition was maintained during the period of conditioning stimulation and often outlasted stimulation by $20-30 \mathrm{~min}$. This inhibition was observed in spinalised as well as intact animals, and only partially reversed by naloxone (Chung, Fang et al., 1984). The A $\delta$ fibre group was found to be the most important for producing inhibition of the spinothalamic tract cells, although significant additional effects were also produced by the A $\alpha \beta$ and $\mathrm{C}$ fibre groups. The most effective nerve in producing inhibition amongst those tested was the ipsilateral tibial nerve. Inhibition was progressively less effective when the contralateral sciatic nerve, the ipsilateral median nerve or the contralateral median nerve was stimulated (Chung, Lee, Hori, Endo, \& Willis, 1984). The inhibition produced by peripheral nerve stimulation was, therefore, shown to be segmentally organized.

\subsubsection{Acupuncture and Heterosegmental effects}

The A $\delta$ afferent fibres also synapse with cells in the anterolateral tract which projects to the midbrain and the pituitary-hypothalamic complex (Bowsher, 1998; Cho, Wong, \& Fallon, 2001; Pomeranz, 2001). In clinical acupuncture, local pain relief can often be achieved by needling distal acupuncture points supplied by nerves from totally different segments to the local area. This heterosegmental effect of acupuncture also occurs in rabbits but can be abolished by severing the anterolateral tract of the spinal cord which contains the spinothalamic and spinoreticular tracts (Chen, Jen, Teh, Yao, \& Shu, 1975). 
The anterolateral tract is, therefore, essential for the conveyance of acupuncture impulses to activate further analgesic responses from higher brain centres.

In the midbrain collaterals of the anterolateral tract cells synapse with and excite the cells in the periaqueductal gray, which in turn activates the descending pain inhibitory pathways from the raphe nucleus (Bowsher, 1998; Cho et al., 2001; Pomeranz, 2001). These are the serotoninergic and noradrenergic systems which descend the dorsolateral tract to release serotonin or noradrenaline onto the enkephalinergic interneurons in the spinal dorsal horn and the spinothalamic tract cells, resulting in suppression of pain impulse transmission (Pomeranz, 2001; White, 1999). Within the periaqueductal gray, a somatotopic organisation is thought to exist and this might explain the reason for acupuncture input at a body site producing analgesia in the same or adjacent segments (Soper \& Melzack, 1982). Wang et al. (1990) demonstrated that lesions in the ventral periaqueductal grey led to significant attenuation of analgesia from both low and high frequency electroacupuncture after 4-6 days. In the raphe magnus nucleus it was found that electroacupuncture inhibited the firing rate of the excitatory nociceptive neurons which might contribute to its analgesic effect (Liu, Zhu, \& Zhang, 1986).

Less well understood is the projection of the anterolateral tract cells to the pituitaryhypothalamic complex. It is possible that projections from the periaqueductal gray to the medial centromedian nucleus of the thalamus, and to the posterior and lateral hypothalamus, eventually result in activation of the medial arcuate nucleus of the anterior hypothalamus leading to the release of $\beta$-endorphins from the pituitary (Takeshige et al., 1993). It is known that acupuncture analgesia is associated with increased $\beta$-endorphin in the cerebrospinal fluid and blood (Clement-Jones et al., 1980). 
However, the mechanism through which the pituitary released $\beta$-endorphin takes part in acupuncture analgesia is not clear (Pomeranz, 2001).

\subsubsection{Acupuncture and the Cerebral Cortex and Subcortical Structures}

Functional magnetic resonance imaging of the brain has found acupuncture influenced activities in the cerebral cortex, the limbic system and subcortical structures (Chiu et al., 2001; Cho et al., 1998; Hui et al., 2000). The association of emotional changes with acupuncture treatment and recent discovery of activation of cerebral cortices by acupuncture has led to the hypothesis that acupuncture activates the hypothalamic centres via the higher brain projecting through the limbic system (Campbell, 1999; Cho et al., 1998; Cho et al., 2001). The hypothalamus, together with the pituitary gland executes homeostatic and controlling functions including the balance of humoral and autonomic functions and the release of neurochemicals. This could contribute to the global effect of acupuncture on the whole body (Cho et al., 2001).

\subsubsection{Acupuncture and Diffuse Noxious Inhibitory Control}

Diffuse noxious inhibitory control (DNIC) is a form of counter-irritation via noxious stimuli, which acts to suppress the transmission of pain sensation (Le Bars, Dickenson, \& Besson, 1979). Opioid receptors may be involved in diffuse noxious inhibitory control (Le Bars, Chitour, Kraus, Dickenson, \& Besson, 1981; Willer, Le Bars, \& De Broucker, 1990). Sims (1997) suggested that DNIC involved C fibres and its effects were transient while acupuncture might not necessarily involve $\mathrm{C}$ fibres and the effects were longer lasting. Although Bing et al. (1990) demonstrated similarities between the effects of acupuncture and DNIC in magnitude, time course, topographic non- 
specificity, and opioidergic link, the role of DNIC in acupuncture analgesia has not yet been clearly established (Pomeranz, 2001).

\subsection{Acupuncture and Humoral Mechanisms}

\subsubsection{Acupuncture and Opioids}

The analgesic effects of acupuncture has a slow onset with an induction period of about 30 minutes (Cao, 2002; Research Group of Acupuncture Anesthesia, 1973). The slow onset and exponential decay of the acupuncture effects suggested the involvement of a humoral mechanism in acupuncture analgesia (Han, 1997). Transfusion of cerebrospinal fluid from acupunctured rabbits into the cerebral ventricles of recipient rabbits has been found to raise the pain thresholds in the latter group (Research Group of Acupuncture Anesthesia, 1973). Raised endorphin levels has also been found in the cerebrospinal fluid in humans after acupuncture (Sjolund, Terenius, \& Eriksson, 1977), and the analgesic effect of acupuncture can be reversed by the opioid antagonist naloxone (Mayer, Price, \& Rafii, 1977). Opioid receptors are present either side of the C fibre synapse in the substantia gelatinosa. When these receptors are activated, transmitter release is inhibited presynaptically and the cell membrane is stabilised against depolarisation postsynaptically (White, 1999). Opioids were also found to suppress excitatory but not inhibitory synaptic transmission in the rat spinal cord substantia gelatinosa neurons (Kohno, Kumamoto, Higashi, Shimoji, \& Yoshimura, 1999).

Different families of endogenous opioid peptides reacting with different class of opioid receptors were found to be released by electroacupuncture of different frequencies (Han, 1997). Enkephalins in the spinal cord and $\beta$-endorphin in the brain were released by low-frequency electroacupuncture; serotonin and dynorphin were released by high- 
frequency electroacupuncture (White, 1998). Electroacupuncture using combination of low and high frequencies was found to produce significantly higher analgesic effects than that induced by fixed-frequency stimulation (Chen, Guo, Chang, \& Han, 1994). This is most likely related to more classes of opioid peptides being released.

Conversely, some experiments have shown pain thresholds elevated by acupuncture failed to reverse when naloxone was given (Chapman, Benedetti, Colpitts, \& Gerlach, 1983). This suggested non-opioid mechanism is also involved in acupuncture analgesia.

\subsubsection{Acupuncture and Other Neurotransmitters}

In addition to the opioid peptides, neurotransmitters have also been associated with the response to acupuncture (Bowsher, 1998; Han, 1997; Kho \& Robertson, 1997; Pomeranz, 2001; Sims, 1997). Of these, the key players are serotonin, dopamine, and noradrenaline. Other neurotransmitters which may play a part include acetylcholine, gamma-aminobutyric acid (GABA), cholecystokinin (CCK), and oxytocin.

\subsection{Motor Control and Synaptic Inputs to $\alpha$-motoneurons}

Motor control is organised hierarchically by interconnected areas of the spinal cord, brainstem and the cortex (Guyton \& Hall, 2000). The cortex is the highest level of motor control. The functions of the motor cortex are influenced by nerve signals from the somatosensory system. The motor cortex receives incoming fibres from the somatosensory areas of the parietal cortex, the adjacent frontal cortex, and the visual and auditory cortices. In addition, it also receives somatosensory fibres directly from the thalamus which relays input from cutaneous tactile, joint and muscle afferents (Guyton 
\& Hall, 2000). Inputs from muscles, joints, and skin are known to produce synaptic potentials in the $\alpha$-motoneurons (Rekling et al., 2000). Motor signals are transmitted to the spinal cord directly via the corticospinal tract, which terminate mainly on the spinal interneurons in the intermediate regions of the cord gray matter. The interneurons then synapse with the $\alpha$-motoneurons which cause the muscle to contract (Guyton \& Hall, 2000).

Indirectly, motor signals also descend to the spinal cord through multiple accessory pathways involving the limbic system, the basal ganglia, cerebellum and various nuclei of the brainstem. These indirect motor signals modulate the activities of the spinal motoneurons (Rekling et al., 2000; White, Fung, Jackson, \& Imel, 1996). From the brainstem, neurons in the raphe pallidus and obscurus, the medial reticular formation, the locus coeruleus and subcoeruleus project to the $\alpha$-motoneurons in the spinal cord (Holstege \& Kuypers, 1987). Neurons in the raphe nucleus are found to be either serotonergic or non-serotonergic. Neurons in the locus coeruleus and subcoeruleus are noradrenergic. Other substances including substance P, thyrotrophin-releasing hormone, enkephalin-like peptides, galanin, cholecystokinin, neurokinin A, glutamate or aspartate are present in the raphe nucleus and medial reticular formation. These substances are also found in the nerve fibres and terminals in the ventral horn of the spinal cord (Rekling et al., 2000).

At the spinal level, muscle spindle Ia and group II afferents provide monosynaptic input to the $\alpha$-motoneurons (Brown \& Fyffe, 1981). These $\alpha$-motoneurons also receive synaptic input via recurrent axon collaterals from other $\alpha$-motoneurons, interneurons and propriospinal neurons which relay afferent signals from muscles, joints and skin (Riddell \& Hadian, 2000; Rudomin, Solodkin, \& Jimenez, 1987). Alpha-motoneuronal 
excitability is affected by the presynaptic release of various transmitters acting on postsynaptic receptors on the motoneuron membrane. Some of these transmitters can also exert influence by actions at presynaptic receptors (Rekling et al., 2000). Glutamate, gamma amino-butyric acid, and glycine are the principal transmitters of local premotor and interneurons but are also involved in certain brain stem projections. Glutamate is the main excitatory, and GABA and glycine are the main inhibitory transmitters acting through ionotropic receptors. As mentioned earlier, serotonin, norepinephrine, thyrotrophin-releasing hormone, substance $\mathrm{P}$, and a host of other peptides are the main transmitters in the projections originating in brain stem nuclei and modulate motoneuronal excitability through pre- and postsynaptic actions via metabotropic receptors (Rekling et al., 2000).

An important link between acupuncture and motor control has been provided by the studies on reflex inhibition of spinal motoneurons during muscle fatigue (BiglandRitchie, Dawson, Johansson, \& Lippold, 1986; Hayward, Breitbach, \& Rymer, 1988). Group III and group IV free nerve endings have previously been shown to react vigorously to muscle metabolites accumulating in fatigue and to muscle contraction (Kniffki, Mense, \& Schmidt, 1978; Mense \& Stahnke, 1983). The afferent limb of the fatigue associated inhibitory reflex has subsequently been shown to involve the group III and group IV muscle afferents (Hayward, Wesselmann, \& Rymer, 1991). On the other hand, group III and group IV fibres are also implicated in the conduction of acupuncture stimulation signals to the central nervous system (Kawakita \& Gotoh, 1996; Okada et al., 1996). In addition, both acupuncture and motor control appear to involve similar structures such as the cerebral cortices, the limbic system, the basal ganglia and brainstem structures and the spinal cord. As noted earlier, some neurotransmitters such as serotonin, noradrenaline, GABA, cholecystokinin, 
acetylcholine and neuropeptides might also be involved in both acupuncture and motor control. Thus, it appears that there is a significant commonality between the neural structures involved in acupuncture analgesia and in motor control. It is, therefore, possible that acupuncture can have an effect on $\alpha$-motoneuronal excitability through activation and/or modulation of activities in these structures.

\subsection{Acupuncture and $\alpha$-Motoneuron Excitability}

Acupuncture has been used clinically for a wide variety of conditions including pain, inflammation, allergies, autonomic nervous system dysfunction and others (Cheng, 1987). It has been beneficial in the treatment of conditions associated with abnormal increase in muscle tone, such as stroke (Guo, Zhou, Chen, \& Wang, 1997; Pei et al., 2001; Wong, Su, Tang, Cheng, \& Liaw, 1999), acute torticollis (Wu, 1996), and acute or chronic neck and low back pain (Irnich et al., 2002; Leibing et al., 2002). Pathophysiologically, spasticity in stroke patients has been associated with changes in spinal motoneuron excitability. (Yu, Wang, \& Wang, 1995). A significantly shorter Hreflex recovery time was demonstrated in the paretic limbs of stroke patients compared with the unaffected side and with normal controls, indicating increased $\alpha$-motoneuron excitability in paretic limbs of these patients (Yu et al., 1995). Other studies showed abnormal electromyographic (EMG) activities in the neck in patients with torticollis at rest (Ostergaard, Fuglsang-Frederiksen, Sjo, Werdelin, \& Winkel, 1996), and abnormal recruitment of the multifidus muscle in low back pain patients during coordination exercise (Danneels et al., 2002).

However, there have been only a few studies into the physiological effects of acupuncture on the motor system. The techniques that have been used include 
observation of changes in EMG, stretch reflex, tonic vibration reflex and H-reflex. A significant reduction of lumbar EMG asymmetry in the back muscles of normal subjects was shown after acupuncture stimulation (Tanaka, Leisman, \& Nishijo, 1998). The improvement in asymmetry was found to be associated with a significant reduction in absolute EMG values when the EMG baseline level was high, when compared to the median value, and a significant increase when it was low (Tanaka et al., 1998). This provided some evidence that acupuncture could influence motor control, although the mechanism was not defined.

A reduction of the stretch reflex response in healthy subjects was noted immediately after insertion of the acupuncture needles (Dawson, Lippold, \& Milne, 1987; Milne, Dawson, Butler, \& Lippold, 1985). This effect faded gradually during the 25-min. period of stimulation but extended beyond it (Dawson et al., 1987; Milne et al., 1985). However, in the same experiment, an H-reflex like response was not affected by acupuncture. It was postulated that acupuncture might have caused a depression of fusimotor activity or inhibition of oligosynaptic pathways leading to a reduction in the reflex response (Dawson et al., 1987).

The tonic vibration reflex involves the muscle spindle and the homonymous motor units activated by the muscle spindles, either monosynaptically or polysynaptically (Enoka, 2002). It has been shown that acupuncture has an inhibitory effect on the tonic vibration reflex contraction (Homma, Endo, \& Sakai, 1980). A similar inhibition occurred in the vibratory grasp reflex after acupuncture (Homma \& Homma, 1982). This effect was partially reversible by the administration of naloxone. It was postulated that endogenous opioid was involved in reducing the facilitatory influence of the spinal interneurons on the motoneurons whose excitability was lowered by acupuncture (Homma, Hori, \& 
Yonezawa, 1985; Homma, Nakajima, \& Toma, 1985). Since the inhibitory effect of acupuncture on the tonic vibration reflex was also found to be present in spinal cord injury patients, it was also suggested that part of this inhibitory effect of acupuncture might be mediated via the spinal cord (Takakura et al., 1996). Using similar experimental methods, Kodachi et al. (1987) observed two separate modes of suppression and recovery after acupuncture. From this, it was postulated that the inhibitory effects of acupuncture operated at both spinal and supraspinal levels (Kodachi et al., 1987).

The effect of acupuncture on $\alpha$-motoneuron excitability in stroke patients was examined by $\mathrm{Yu}$ et al. using the H-reflex recovery time (Yu et al., 1995). Acupuncture was found to prolong the shortened H-reflex recovery time in the limbs on the paretic side and approximate the H-recovery curve of the paretic limb to that of the normal controls. Yu et al. (1995) argued that the increased $\alpha$-motoneuron excitability in paretic patients could be from either excessive excitation or decreased inhibition at a segmental level, and since acupuncture treatment decreased the $\alpha$-motoneuron excitability, they concluded that their experiments established the effect of acupuncture at the spinal level.

The H-reflex was also used by Chang et al. (2001) to compare the extra-segmental effect effects of manual acupuncture and transcutaneous electrical nerve stimulation (TENS) on the spinal cord in healthy subjects. They found both low and high frequency TENS increased excitability of the $\alpha$-motoneurons but manual acupuncture had no effect. 
It appears that the available evidence from the few studies indicates that acupuncture has an inhibitory effect on the reflexes under question. However, the evidence is scanty and disparate, leaving many unanswered questions. Parameters of treatment for optimal effect on the motor system have not been examined and strong evidence as to the effect of acupuncture on muscle activity is lacking. Few studies have looked at the subjective sensation of deqi, an important clinical phenomenon, to assess its value in the physiological sense.

\subsection{The Hoffmann Reflex Methodology}

The Hoffmann-reflex is an artificially induced response evoked by stimulating a mixed peripheral nerve to a muscle (Magladery \& McDougal, 1950) (Fig.1.1). The Ia afferent fibres in the nerve are stimulated preferentially at low intensities. These synapse essentially monosynaptically with homonymous $\alpha$-motoneurons in the anterior horn of the spinal cord. The impulse generated is then transmitted in the motor axon to the recipient muscle which responds with a contraction. The electromyogram (EMG) of this muscle contraction elicited by the mixed peripheral nerve stimulation is called the Hoffmann-reflex (H-reflex) (Hugon, 1973; Magladery \& McDougal, 1950). With increasing intensity of stimulation, an increased number of $\alpha$-motoneurons excited is reflected in an increase in the H-reflex amplitude. At higher intensity of stimulation, the motor axons in the peripheral nerve are also excited and these evoke direct stimulation of the muscle endplates, leading to the appearance of a second wave in the EMG. This is at a shorter latency than the H-reflex and is called the M-wave (Magladery \& McDougal, 1950). In simple terms, the M-wave involves stimulation of the motor axons, the neuromuscular junctions, and the muscle. It can be affected by the state of these anatomical areas and the stimulation site itself. The amplitude of the H-reflex, in 
addition, involves the afferent Ia fibres, their synapses with the $\alpha$-motoneurons, and the excitability of the $\alpha$-motoneurons themselves. The amplitude is also influenced by the state of these anatomical areas and the stimulation site. In the H-reflex methodology for assessing $\alpha$-motoneuron excitability after an intervention, $\mathrm{M}$-waves are recorded before and after the intervention to ensure peripheral stability, so that any changes in the $\mathrm{H}$ reflex can be ascribed to changes in the central nervous system (Bradnam, Rochester, \& Vujnovich, 2000; Funase, Imanaka, \& Nishihira, 1994; Kerr, Vujnovich, \& Bradnam, 2002b).

The H-reflex is a useful tool for investigating changes in the excitability of the $\alpha$ motoneuron pool (Pierrot-Deseilligny \& Mazevet, 2000). Different parameters have been used in the H-reflex methodology to assess $\alpha$-motoneuron excitability. These include the H-reflex recovery time after a conditioning stimulus (Taborikova \& Sax, 1969; Schieppati, 1987), the ratio between the maximum amplitude of the H-reflex and the M-wave $\left(\mathrm{H}_{\max } / \mathrm{M}_{\max }\right)$ (Casabona, Polizzi, \& Perciavalle, 1990; Dishman \& Bulbulian, 2001), the ratio of the threshold of the H-reflex to the threshold of the Mwave $\left(H_{t h} / M_{t h}\right)$ (Davies \& Lader, 1985), the slope of the H-reflex recruitment curve $\left(\mathrm{H}_{\text {slp }}\right)$ (Bradnam et al., 2000), and the ratio of the slope of the H-reflex recruitment curve to the slope of the M-wave recruitment curve $\left(\mathrm{H}_{\mathrm{slp}} / \mathrm{M}_{\text {slp }}\right)$ (Funase, Higashi, Yoshimura, Imanaka, \& Nishihira, 1996; Funase et al., 1994). 


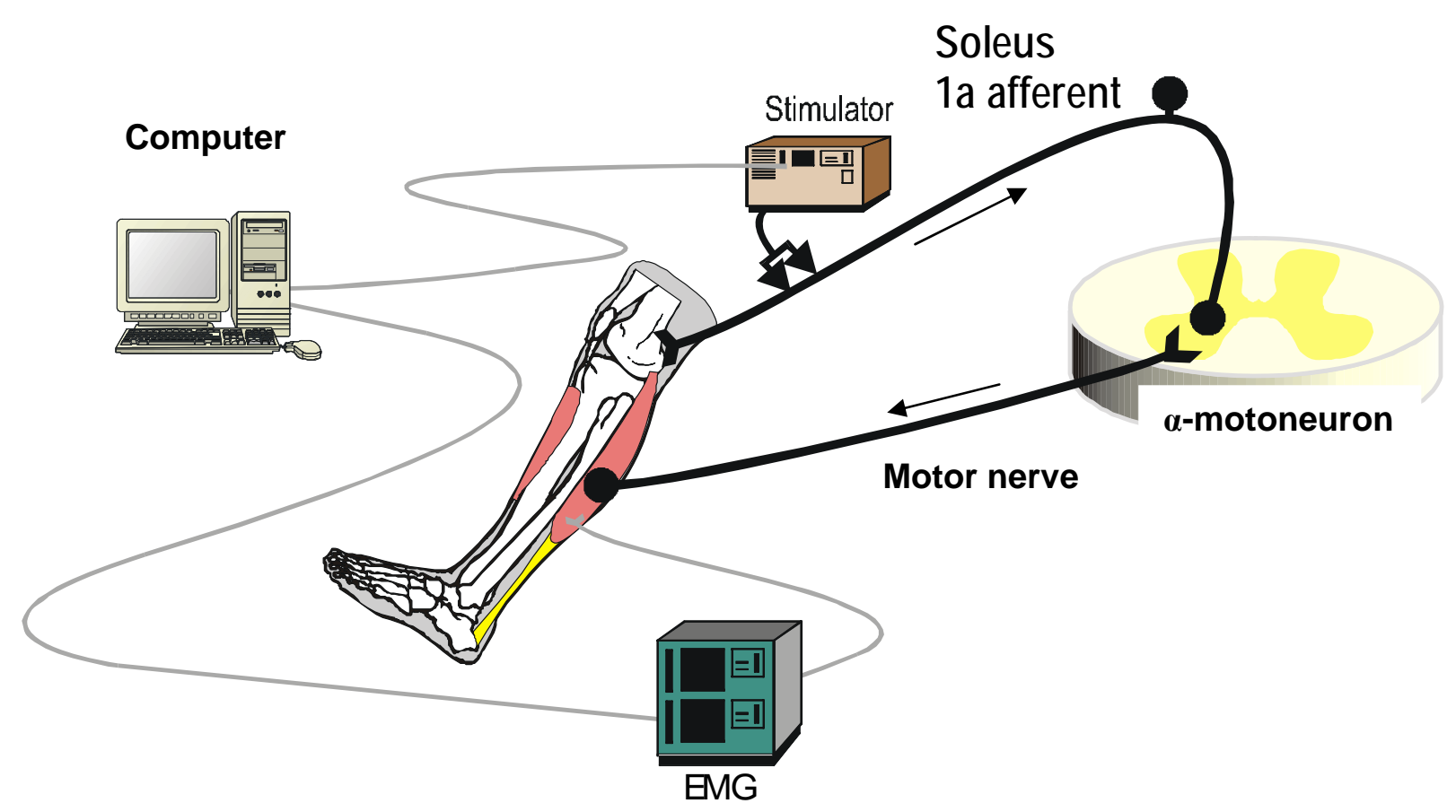

Fig.1.1 The H-reflex stimulation and recording system. A computer programme triggers the stimulator to cause an electrical stimulation of the Soleus la afferent fibres. These fibres synapse essentially monosynaptically with the homonymous $\alpha$-motoneurons in the spinal cord, resulting in an impulse traveling down the motor nerve to the Soleus muscle which then contracts. The contraction of the Soleus muscle is recorded with EMG electrodes and stored in the computer for further analysis. (Courtesy of Jill Kerr and Steve Stanley). 


\subsection{Purpose of the Study}

H-reflex methodology has been used to assess the effects of therapeutic interventions such as spinal manipulation, massage, sacro-iliac joint manipulation, cervical traction, tendon pressure and transcutaneous electrical nerve stimulation (Bradnam et al., 2000; Chang et al., 2001; Dishman \& Bulbulian, 2001; Dishman, Cunningham, \& Burke, 2002; Hardy et al., 2002; Kukulka, Beckman, Holte, \& Hoppenworth, 1986; Morelli, Seaborne, \& Sullivan, 1991; Morelli, Sullivan, \& Chapman, 1998; Murphy, Dawson, \& Slack, 1995). There have only been two previous studies investigating the effect of acupuncture on $\alpha$-motoneuron excitability in normal subjects (Chang et al., 2001; Hsieh, 2002) and one study in stroke patients (Yu et al., 1995). None of these have assessed adequately the effects of segmental acupuncture, the parameters of treatment duration and subjective intensities of deqi in relation to the outcome measurements.

The purpose of the present study was to use the H-reflex methodology to

1. Evaluate the effect of acupuncture on $\alpha$-motoneuron excitability when given ipsilaterally and in the same segment.

2. Compare the effect of short and long duration acupuncture.

3. Evaluate the correlation between the intensity of acupuncture sensation (deqi) and the effect of acupuncture.

The knowledge gained will assist in the provision of optimal treatment duration and intensities in clinical practice and help in providing evidence-based practice for acupuncture. 


\section{Chapter $2 \quad$ Methodology}

\subsection{Objectives of the Study}

The aim of the study was to examine the effect of acupuncture on the $\alpha$-motoneuron pool excitability. Specifically, the acupuncture needles were inserted into two acupuncture points related to the $\mathrm{S} 1$ segment. The H-reflex has been widely used by different researchers for the assessment of changes in motoneuron excitability because it is considered to provide a sensitive measure (Goldberg, Sullivan, \& Seaborne, 1992; Higashi et al., 2001; Hilgevoord, Koelman, Bour, \& Ongerboer de Visser, 1994; Hopkins, Ingersoll, Edwards, \& Cordova, 2000; Miglietta, 1970; Rochester, Vujnovich, Newstead, \& Williams, 2001; Sullivan, Williams, Seaborne, \& Morelli, 1991; Vujnovich \& Dawson, 1994). The Soleus H-reflex, which measures the S1 motoneuron pool excitability, was employed for the same purpose in the present study. The second aim of this study was to investigate the difference in effect from different duration of acupuncture. The third aim of the study was to observe if there was any correlation between the intensity of perceived acupuncture sensation (deqi) and the effect of acupuncture.

\subsection{Hypotheses}

It is hypothesised that

1) Segmental acupuncture at $\mathrm{S} 1$ level will result in a reduction in the $\alpha$-motoneuron pool excitability in the same segment. 
2) The effect on the $\alpha$-motoneuron pool excitability from 5 minutes (short duration) acupuncture will be less than that from 20 -minutes (sustained) acupuncture, both in intensity and in duration.

3) A higher intensity of perceived acupuncture sensation (deqi) is correlated with greater suppression of $\alpha$-motoneuron pool excitability.

\subsection{Methods}

\subsubsection{Introduction}

The effects of short duration acupuncture and sustained acupuncture on the $\alpha$ motoneuron pool of the Soleus muscle were assessed by the H-reflex technique in 20 asymptomatic, neurologically normal subjects. The experiments were carried out in a quiet environment within the Human Neurophysiology Laboratory of the Physical Rehabilitation Research Centre, Auckland University of Technology, where all unnecessary sounds or movements were kept to the minimum.

\subsubsection{Selection of test subjects}

Subjects were invited to participate on a voluntary basis. Subjects were excluded from the study if they had a history of previous spinal surgery, systemic disease, neurological disease, or persistent pain in the lower back or in the lower limbs.

Subjects were requested to abstain from taking aspirin, caffeine or alcohol (Eke-Okoro, 1982) and vigorous exertion (Bulbulian \& Darabos, 1986) on the morning of the day of the experiment. 
Ethical approval was obtained from the Auckland University of Technology Ethics Committee. Written and verbal explanations of experimental procedures were provided to subjects prior to testing. Each subject signed an informed consent form.

\subsubsection{Stimulating and recording of H-reflex and M-waves}

\subsubsection{Subject Position}

During the experimental procedure, subjects were seated in a dental chair with adjustable back, leg and foot supports. To control for the known effect of muscle contraction or change in joint position on the H-reflex (Chapman, Sullivan, Pompura, \& Arsenault, 1991; Hugon, 1973), special attention was paid to the positioning of the subjects. The back of the chair was fixed in a semi-reclining position to allow for comfort required for a prolonged period of experimentation. The seat of the chair was fixed at an angle of $120^{\circ}$ to the leg support. As unrestrained ankle could lead to greater variation in serial recording of the H-reflexes, the leg of the subject was strapped to the leg support (Little, Hayward, \& Halar, 1989). The leg was maintained at an angle of $80^{\circ}$ to the foot with a foot-rest. The head of the subject was positioned on a head rest, and the subject was asked to keep the head still in the midline during the experiment.

\subsubsection{Skin Preparation}

The skin was prepared for optimal electrical conduction prior to placement of the electrodes. Surface cells were removed by light abrasion with Omni Prep ${ }^{\circledR}$ (D.O. Weaver \& Co., Aurora, USA), and the area was then cleaned with alcohol and rubbed dry with soft paper tissues to remove any residual abrasive paste. Surface electrodes were then attached for stimulating and recording the H-reflexes and M-waves. 


\subsubsection{Stimulating Electrodes Placement}

To stimulate the tibial nerve, two 9-mm Silver/Silver Chloride disc electrodes coated with Ten 20 Conductive EEG Paste (D.O. Weaver \& Co., Aurora, USA) were used as cathode and anode. The cathode was positioned over the tibial nerve at the popliteal crease and secured with Fixomull ${ }^{\circledR}$ Stretch Tape (Beiersdorf AG, Hamburg, Germany). This stimulating electrode was further fixed at the popliteal fossa with a piece of foam held by elastic bandage. The anode was positioned on the knee just lateral to the patella using Fixomull ${ }^{\circledR}$ Stretch Tape. Placement of the stimulating electrode was considered to be adequate when a threshold stimulus elicited an H-reflex without any direct Mwave (Hugon, 1973).

\subsubsection{Recording Electrodes and Earthing Electrode Placement}

The H-reflexes and M-waves were recorded with a disposable self-adhesive bipolar EMG bar electrode (Noro-trode TM, Naromed Inc., Seattle, WA.). The position of the recording electrode was determined by measuring the distance between the popliteal crease and the Achilles tendon insertion. The recording electrode was placed in the midline of the Soleus muscle parallel to the muscle fibres, three quarters of the distance distal from the popliteal crease (Maryniak \& Yaworski, 1987). The electrode was determined to be correctly placed when the H-reflex had a similar configuration to the M-wave (Hugon, 1973; Sabbahi \& Khalil, 1990).

A single disposable self-adhesive earth electrode (3 M Healthcare, St. Paul, MN.) was positioned over the anterolateral tibia at the same level as the recording electrode. 


\subsubsection{Equipment used for Stimulating and Recording}

Regular and sequential series of signals were generated with the LabVIEW computer software (National Instruments Inc., USA) and fed into a Digitimer DS7 constant current stimulator (Digitimer Ltd, Hertfordshire, UK) which was used to deliver the stimulating electrical impulses to the popliteal nerve.

The H-reflexes and M-waves elicited in the Soleus muscle were recorded electromyographically after amplification and filtering with a Grass P5 Series Amplifier (Grass Instrument Co., USA).

\subsubsection{Stimulation and Recording of Reflex Responses}

The Digitimer DS7 stimulator delivered a $0.5-1.0 \mathrm{~ms}$ duration square wave pulse to the tibial nerve. This impulse preferentially activates the large sensory fibres (Panizza, Nilsson, Roth, Basser, \& Hallett, 1992). LabVIEW computer software triggered the Digitimer DS7 stimulator regularly every 6 seconds to deliver the stimulation impulses (Hugon, 1973). The intensity of stimulation was increased stepwise from below the threshold for eliciting an H-reflex $\left(\mathrm{H}_{\mathrm{th}}\right)$ to that producing maximum $\mathrm{M}$-wave amplitude $\left(\mathrm{M}_{\max }\right)$. At each specific intensity, the stimulation was repeated three times. The electromyographic signals generated in the Soleus muscle were sampled at a rate of 5 $\mathrm{kHz}$, then amplified and filtered with a bandwidth of $1 \mathrm{~Hz}-5 \mathrm{kHz}$ by a Grass P5 Series Amplifier. LabVIEW computer software was used to display digitised data on the computer screen for direct scrutiny. Recordings were stored and the peak-to-peak amplitudes of the H-reflexes and M-waves were later analysed. 


\subsubsection{Acupuncture needles}

Sterile, single use disposable acupuncture needles with a gauge of $20 \times 40$ and with guide-tubes (Suzhou Medical Instruments, Suzhou, China) were used.

\subsubsection{H-reflex and M-wave Recordings}

$\mathrm{H}$-reflexes and $\mathrm{M}$-waves in response to the stepwise increase in stimulus intensity were recorded and their peak-to-peak amplitudes were measured using mobile measuring cursors in the LabVIEW computer software. The same peaks were used throughout for each individual H-reflex and M-wave within the same trial. The first trial data were not included in the statistical analysis as the trial was performed to familiarise the subjects to the stimulus intensities to be used in the experiment. The rate of change in the $\mathrm{H}$ reflex amplitude between threshold and maximum was also assessed in the first trial. This information allowed finer adjustment of the range of stimulus intensities so that the ascending slope of the H-reflex recruitment curve could be constructed more accurately. The relationship between the amplitudes of the H-reflexes or M-waves and the stimulus intensities was later represented graphically by their respective recruitment curves.

\subsubsection{Criteria for Acceptance of an H-Reflex}

At low stimulus intensities, the largest diameter and lowest threshold Ia afferent fibres of the tibial nerve were recruited and an H-reflex was elicited. The Soleus H-reflex was taken as the first deflection from the baseline occurring 21-38 msec after the stimulus artefact (Maryniak \& Yaworski, 1987; Sabbahi \& Khalil, 1990).

Standard criteria were used for accepting the reflex recorded as an H-reflex (Fisher, 1992; Hugon, 1973; Jabre, 1981).

(1) The H-reflex appeared at the correct latency following the stimulus artefact. 
(2) The H-reflex started to appear at stimulus intensities below the threshold of the Mwave.

(3) The size of the H-reflex increased as the stimulus intensities increased and reached a maximum soon after M-threshold.

(4) The H-reflex decreased in amplitude when the M-wave amplitude started to increase and finally disappeared when the M-wave amplitude was around maximum.

\subsubsection{Criteria for Determination of $\mathrm{H}$-threshold $\left(\mathrm{H}_{t h}\right)$}

The lowest stimulating current intensity that evoked an H-reflex in three out of six stimuli was determined to be the H-reflex threshold intensity (Davies \& Lader, 1985). The H-reflex threshold $\left(\mathrm{H}_{\mathrm{th}}\right)$ is the smallest $\mathrm{H}$-reflex amplitude measurable and reflects the smallest number of motoneurons in the $\alpha$-motoneuron pool activated.

\subsubsection{3 $\underline{\text { Criteria for Determination of maximum H-reflex }\left(\mathrm{H}_{\max }\right)}$}

The stimulating current intensity that evoked the largest H-reflex peak-to-peak amplitude was determined to be the maximum H-reflex intensity and the resultant $\mathrm{H}$ reflex the maximum H-reflex $\left(\mathrm{H}_{\max }\right)$.

\subsubsection{Criteria for Determination of M-threshold $\left(\mathrm{M}_{\underline{t h}}\right)$}

The Soleus M-wave was taken as the deflection from the baseline occurring 10-15 sec after the stimulus artefact (Murphy et al., 1995). $\mathrm{M}_{\text {th }}$ was defined as the first deflection from the baseline after the stimulus artefact at this latency. 


\subsubsection{Criteria for Determination of $\mathrm{M}$-maximum $\left(\mathrm{M}_{\max }\right)$}

$\mathrm{M}_{\max }$ was defined as the $\mathrm{M}$-wave with the largest peak-to-peak amplitude to which further increase in stimulus intensity would not lead to any further changes (Hugon, 1973). $M_{\max }$ indicates that the maximal number of motor axons has been stimulated.

\subsubsection{Recruitment Curve Recording Protocol}

Reflexes were recorded in response to progressive increase in stimulus intensity over 14 steps from pre-threshold H-reflex to maximal M-wave. The stimulus intensities for $\mathrm{H}_{\mathrm{th}}$, $H_{\max }, M_{t h}$ and $M_{\max }$ were initially determined by gradually increasing the stimulus intensity while the H-reflexes and M-wave amplitudes were monitored on the computer screen. $H_{\text {th }}, H_{\max }, M_{t h}$ and $M_{\max }$ were then recorded according to the definitions above. From these, a guide to the range of 14 stimulus intensities to be used for the H-reflex and M-wave recruitment curves were calculated $\left(-25 \%,-15 \%, \mathrm{H}_{\text {th }}=0 \%,+15 \%,+25 \%\right.$, $\left.+35 \%,+45 \%,+55 \%,+85 \%, \mathrm{H}_{\max }=100 \%,+115 \%,+150 \%, \mathrm{M}_{\max },+110 \% \mathrm{M}_{\max }\right)$ and drafted on the data sheet. The distribution of the percentage figures used in the calculation was based on the finding that facilitation of H-reflex usually reached a maximum at around $30 \%$ of $\mathrm{M}_{\max }$ and inhibition of $\mathrm{H}$-reflex reached a maximum at around $40-50 \%$ of $\mathrm{M}_{\max }$ (Crone, Hultborn, Mazieres, Nielson, \& Pierrot-Deseilligny, 1990). Further adjustment of the final range of stimulus intensities depended on the actual shape of the individual's H-reflex recruitment curve in the first trial. The aim was to allow more data points to be created in the ascending slope of the recruitment curves. Because of variability in the H-reflex amplitude at the same stimulus intensity (Funase \& Miles, 1999), at each intensity level three stimuli were given at an interval of 6 seconds, and the responses were all recorded. 


\subsubsection{Experimental Design}

The effects of two acupuncture interventions of different duration were compared using a repeated-measures, crossover design.

Each subject received both interventions. To minimise any carry-over effects, the interventions were delivered at least two weeks apart. To control for the learning effect, the order of short duration acupuncture and sustained acupuncture was randomised for each subject.

At each experiment, two H-reflex and M-wave recruitment curves (Trials 1 and 2) were recorded 5 minutes apart before the randomised acupuncture intervention. These were used as controls. Further trials were then performed to assess the change in $\alpha$ motoneuron excitability immediately after acupuncture (Trial 3) and 15 minutes after acupuncture (Trial 4). A proportion of participants had another recording 30 minutes after acupuncture (Trial 5) to assess the time course of the acupuncture effect. The experimental protocol is summarised in Table 2.1 . 
Table 2.1 The experimental protocol used in the study. For the purpose of presentation and discussion, pre-acupuncture trials are referred to as Trial 1 and Trial 2, while post-acupuncture trials are referred to as Trial 3, Trial 4 and Trial 5.

\begin{tabular}{|l|l|}
\hline Trial 1 & Pre-acupuncture trial 1 (Control) \\
\hline Trial 2 & Pre-acupuncture trial 2 (Control) \\
\hline & INTERVENTION \\
& (ACUPUNCTURE - Short Duration or Sustained) \\
\hline Trial 3 & Post-acupuncture trial immediately on withdrawal of needles \\
\hline Trial 4 & Post-acupuncture trial 15 minutes after withdrawal of needles \\
\hline Trial 5 & Post-acupuncture trial 30 minutes after withdrawal of needles \\
\hline
\end{tabular}




\subsubsection{Acupuncture points used}

The acupuncture points GB34 (Yanglingquan) and SP9 (Yinlingquan) were selected for this study. It was expected that acupuncture at these two sites would cause afferent impulses to travel to the L4, L5, S1 and S2 segments of the spinal cord and higher centres within the nervous system. As a result, the excitability of the $\alpha$-motoneuron pool of those segments would be affected. These changes would be reflected in the difference in the H-reflex recruitment curves before and after the acupuncture interventions.

\subsubsection{GB34 (Yanglingquan)}

GB34 is located in the lateral aspect of the knee in the depression anterior and inferior to the head of the fibula (Cheng, 1987). The point can be punctured perpendicularly for 0.8 to 1.2 inches. It is close to the area where the common peroneal nerve bifurcates into the superficial and deep peroneal nerve. A needle inserted into GB34 will pass through the Peroneal Longus, Extensor Digitorum Longus, and Tibialis Anterior muscles. Peroneal Longus and Extensor Digitorum Longus have nerve supply from L5 and S1, and Tibialis Anterior has nerve supply from L4 and L5 (Williams et al., 1995).The nerve supply of the skin in the area is within the territory of the L5 dermatome.

\subsubsection{SP9 (Yinlingquan)}

SP9 is located on the lower border of the medial condyle of the tibia, in the depression on the medial border of the tibia (Cheng, 1987). The point can be punctured perpendicularly 0.5 to 1.0 inch. The point comes close to the medial crural cutaneous nerve superficially and the tibial nerve in the deeper area. A needle inserted into SP9 will pass through the Gastrocnemius and Soleus muscles. The Gastrocnemius and the Soleus muscles have nerve supply from S1 and S2 (Williams et al., 1995). The nerve supply of the skin in the area is within the territory of the L4 dermatome. 


\subsubsection{Acupuncture procedure}

The skin over GB34 and SP9 was cleaned with 70\% ethyl alcohol, and the acupuncture needles were inserted perpendicularly. The subject was asked to indicate when acupuncture sensation (deqi) occurred. "Deqi" is defined as "the arrival of qi and refers to soreness, numbness or a distending feeling around the point after the needle is inserted to a certain depth. At the same time the operator may feel tenseness around the needle" (Cheng, 1987).

At each acupuncture point, the needle was manipulated to maintain the acupuncture sensation for at least 1 minute. The needles were then left in place. For the short duration acupuncture intervention, no further manipulation of the needles was undertaken. The needles were removed 5 minutes after the first arrival of acupuncture sensation (deqi). For the sustained acupuncture intervention, the needles were manipulated at 5 minutes intervals. On each occasion, acupuncture sensation was maintained for at least 1 minute. The needles were removed 20 minutes after the first arrival of acupuncture sensation (deqi).

\subsection{H-reflex and M-wave Recruitment Curves}

\subsubsection{Averaging of Response Amplitudes}

The peak-to-peak amplitudes of all H-reflexes and M-waves and corresponding stimulus intensities including $\mathrm{H}_{\text {th }}, \mathrm{H}_{\max }, \mathrm{M}_{\text {th }}$ and $\mathrm{M}_{\max }$ were transferred to the Excel software (Microsoft ${ }^{\circledR}$ Excel 2002 SP-1, Copyright (C Microsoft Corporation, 1985-2001). This allowed organisation of the data for graphical display and linear regression analysis. 
The response amplitudes of $\mathrm{H}$-reflexes and $\mathrm{M}$-waves to three consecutive stimuli of the same intensity were averaged to reduce variation on the respective recruitment curves so that the most linear ascending part of the curves could be assessed. The results of linear regression analysis were found to be not affected by selecting points from the averaged response graphics comparing to that when the raw data were used (Refer section 2.6.1).

Graphics showing the different visual impact of the raw data and averaged data on the recruitment curves from the same trial are presented in Figures 2.1A and 2.1B. 


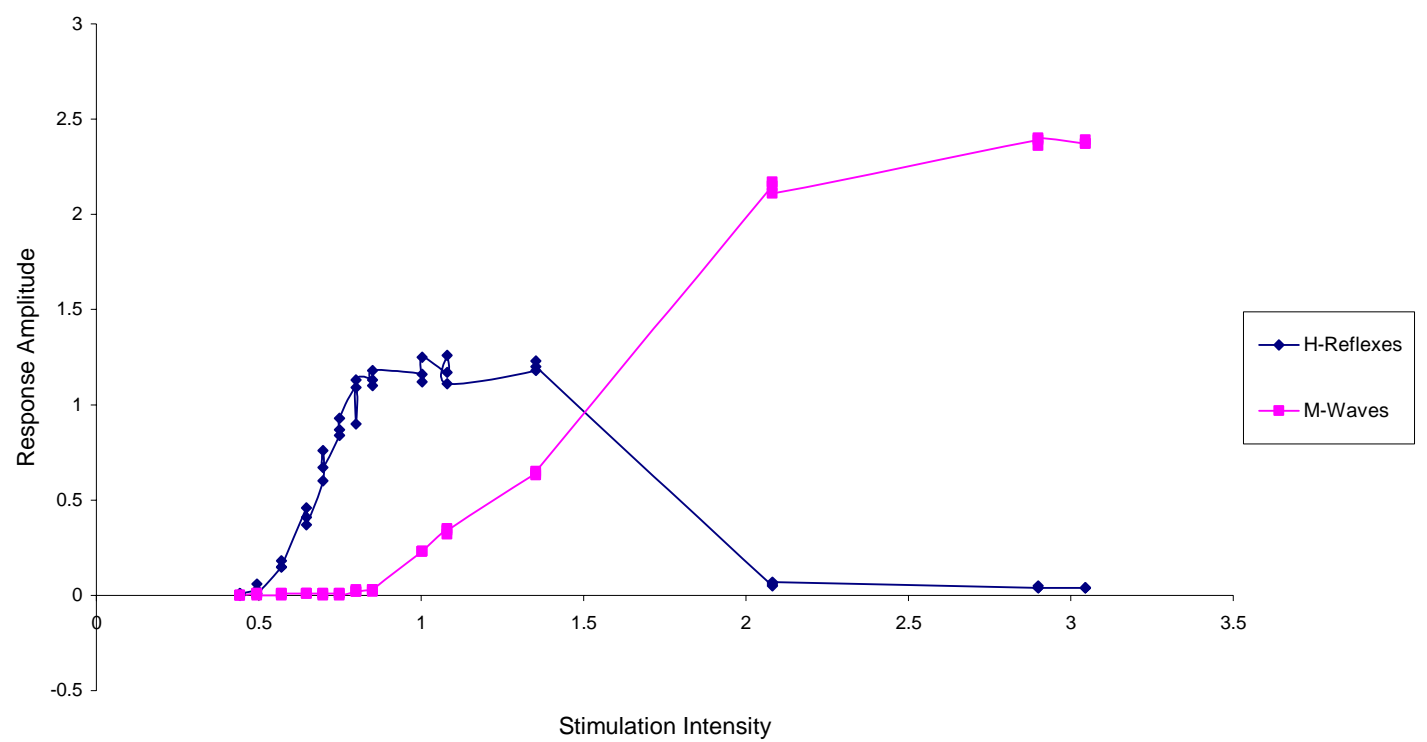

Fig. 2.1A H-Reflex and M-Wave Recruitment Curves constructed from raw data. The variation on the ascending slope, especially in the H-reflex recruitment curve, does not allow easy fitting of a linear regression line.

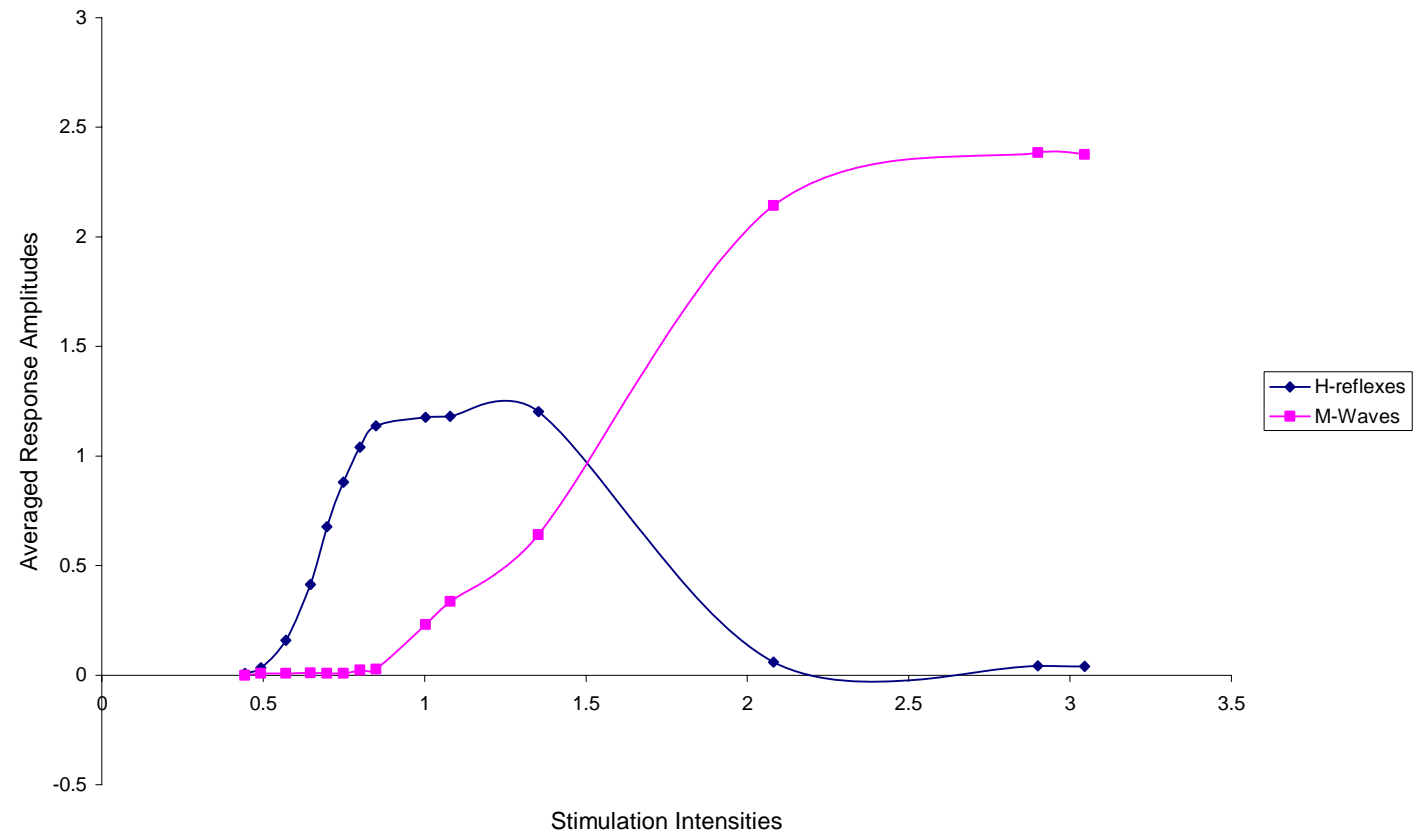

Fig. 2.1B H-reflex and M-wave Recruitment Curves constructed from averaged amplitudes. Each data point represents the average of three response amplitudes at the same stimulus intensity. It is much easier to fit a linear regression line to the averaged recruitment curves. 


\subsubsection{Data Normalisation}

To reduce inter-individual differences and variability in absolute H-reflex and M-wave sizes due to peripheral factors, and to allow data to be grouped for between-subjects statistical analysis, amplitudes of H-reflexes and M-waves and the corresponding stimulus intensities were normalised according to established protocol (Funase et al., 1996; Funase et al., 1994; Hilgevoord et al., 1994; Mazzocchio, Scarfo, Mariottini, Muzii, \& Palma, 2001).

H-reflexes and M-waves were normalised by expressing peak-to-peak amplitudes as a percentage of the averaged maximum $M$-wave $\left(\mathrm{M}_{\max }\right)$ over the four or five trials. According to Hugon (1973) the maximum M-wave $\left(M_{\max }\right)$ represents excitation of all the motor fibres of the nerve to the Soleus muscle and therefore, the activity of $100 \%$ of the Soleus motoneuron pool. Representing data as a percentage of $\mathrm{M}_{\max }$ revealed changes in excitability of motoneurons relative to the entire motoneuron pool. Data were expressed according to the following equation:

Normalised H-reflex (or M-wave) amplitude $=\frac{\text { Mean Amplitude }}{\text { Mean } \mathrm{M}_{\max }} \times 100$

The normalised H-reflex amplitude reflects the percentage of the $\alpha$-motoneurons from the possible total pool causing the maximal contraction of the muscle. Also, expressing the H-reflex or $\mathrm{M}$-wave amplitude as a percentage of $\mathrm{M}_{\max }$ helps to get rid of the changes in muscle geometry related to muscle length and contraction (PierrotDeseilligny \& Mazevet, 2000). 
The stimulus intensities were normalised by expressing each intensity as a multiple of the $M$-wave threshold intensity $\left(\mathrm{M}_{\mathrm{th}}\right)$. Here, $\mathrm{M}_{\mathrm{th}}$ was always expressed as 1 . The following equation was used:

Normalised Stimulus Intensity $=\frac{\text { Stimulus Intensity }(\mathrm{mA})}{\mathrm{M}-\text { Threshold Intensity }\left(\mathrm{M}_{\mathrm{th}} \text { in } \mathrm{mA}\right)}$

\subsubsection{Plotting of Recruitment Curves}

Recruitment curves were plotted with normalised, averaged H-reflex or M-wave amplitudes on the $y$-axis and normalised stimulus intensities on the $x$-axis for all experiments. Thus, each point on the H-reflex recruitment curve reflected the percentage of motoneurons in the $\alpha$-motoneuron pool excited by particular unit of stimulus intensity relative to that producing a threshold M-wave for that particular subject. Similarly, each point on the M-wave recruitment curve reflects the percentage of motor axons excited by a particular unit of stimulus intensity relative to $M_{\text {th }}$.

An example of normalised recruitment curves from a participant is presented in Fig. 2.2. On the $x$-axis, the stimulus intensity evoking $\mathrm{M}_{\mathrm{th}}$ is represented by 1.00 . On the $y$-axis the maximum M-wave is represented by 100 . 


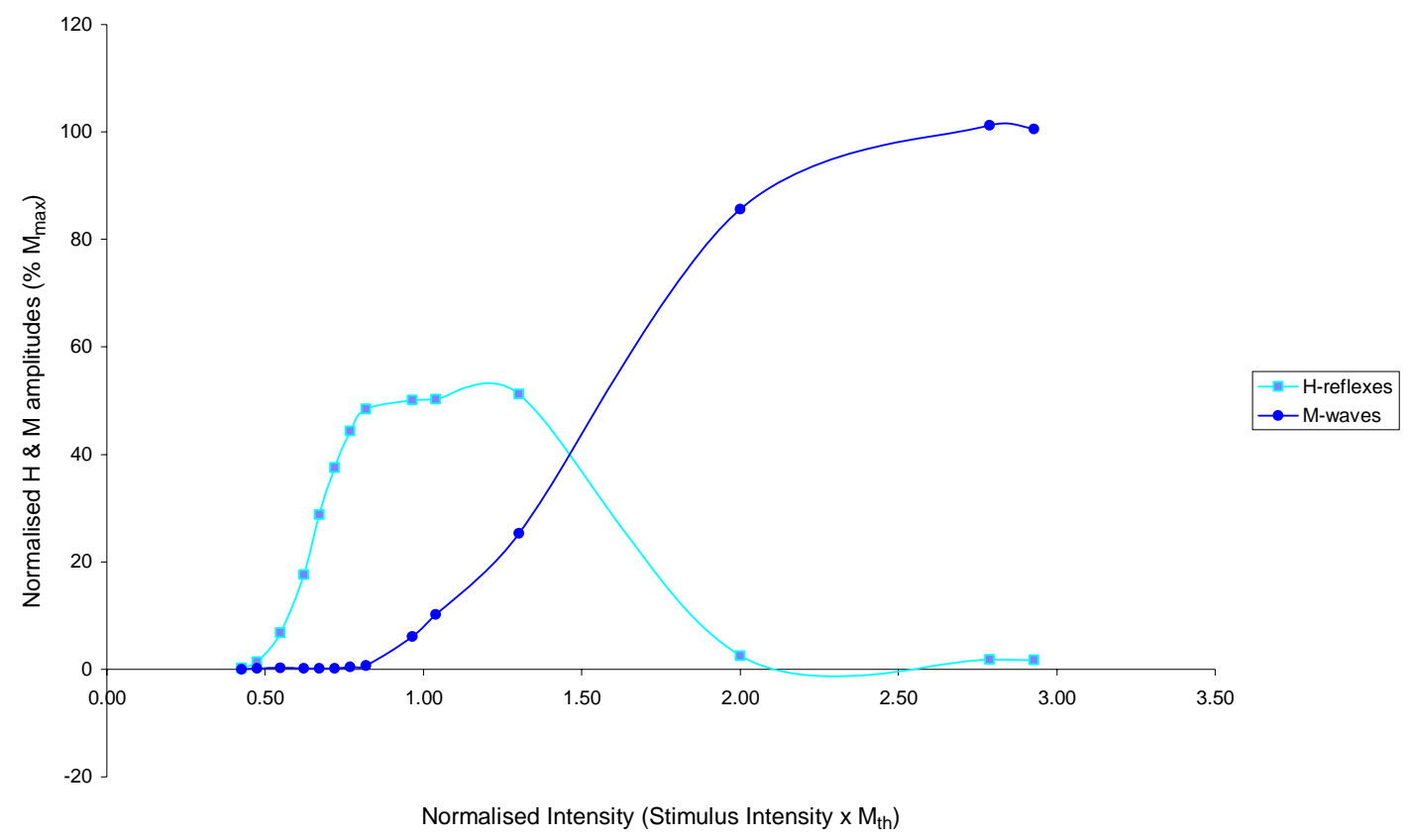

Fig. 2.2 Normalised H-Reflex and M-Wave Recruitment Curves. Each data point represents the average of 3 response amplitudes at the same stimulus intensity. 


\subsubsection{The H-reflex Recruitment Curve}

The H-reflex recruitment curve is characteristically bell-shaped, which represents the increase in the number of $\alpha$-motoneurons activated with each increment of stimulus intensity. At higher stimulus intensities, direct stimulation of the motor axons causes an M-wave to appear. This also leads to propagation of antidromic impulses along the same motor axon to the $\alpha$-motoneurons causing collision with the orthodromic impulses from reflex stimulation via the Ia afferents. This results in reduction in the amplitudes of H-reflexes until they are completely extinguished (Fisher, 1992; Hugon, 1973). With increasing stimulus intensities, excitation of inhibitory interneurons, Renshaw cells, direct motoneuron to motoneuron connections and central inhibition (Fisher, 1992) may also contribute to H-reflex inhibition.

\subsubsection{The M-wave Recruitment Curve}

The M-wave recruitment curve is characteristically sigmoid-shaped. The largest motor fibres are excited first, but because they are fewer in numbers, the M-waves are of smaller amplitudes. As the intensity of stimulation increases, the higher thresholds fibres are also excited. These are more numerous and the curve rises rapidly. Lastly, with further increase in stimulus intensity, the smallest motor fibres of the highest threshold are excited. As these are even less numerous, the curve rises more slowly (Hugon, 1973). 


\subsection{Linear Regression Analysis of H-reflex \& M-Wave Recruitment}

\section{Curves}

From the normalised H-reflex recruitment curve it can be seen that the ascending slope depicts a linear relationship between the increase in amplitude of the H-reflex and the increase in stimulus intensity. Indirectly, the recruitment curve reflects the number of motoneurons firing in response to the specific intensity of stimulation. Thus, it can be inferred that as stimulus intensity is increased, additional $\alpha$-motoneurons are being recruited. The slope of this part of the recruitment curve can, therefore, indicate the rate of recruitment of additional $\alpha$-motoneurons with increase in stimulus intensity. This rate of recruitment reflects the excitability of the $\alpha$-motoneuron pool.

Linear regression analysis was applied to the linear portion of the ascending part of the H-reflex recruitment curve using EXCEL (Microsoft @ Excel 2002 SP-1, Copyright (C) Microsoft Corporation, 1985-2001). The linear regression equation was:

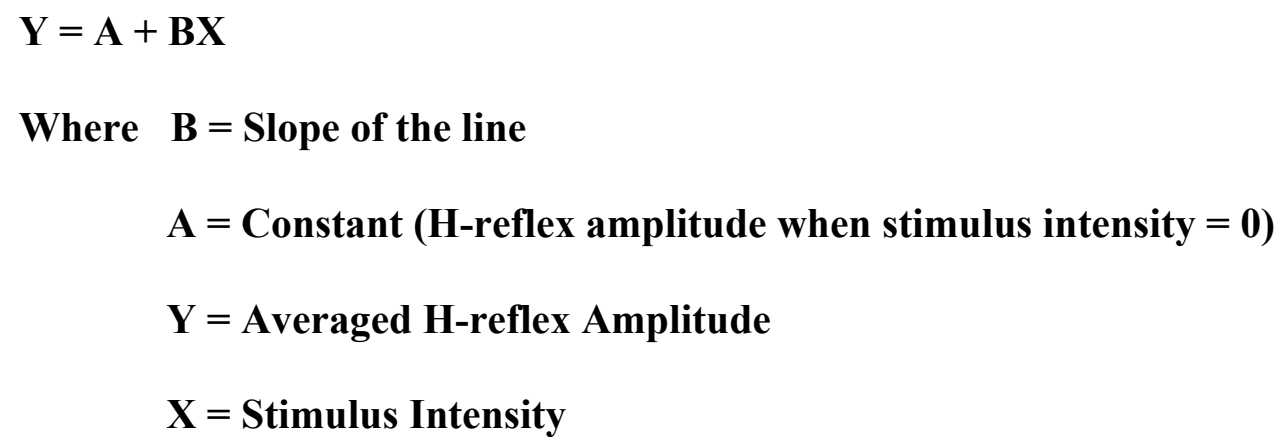

The slope of the line (B) described the increase in H-reflex amplitude per unit increase in stimulus intensity and was called $\mathrm{H}$-slope $\left(\mathrm{H}_{\mathrm{slp}}\right)$. A change in the ascending slope of the H-reflex recruitment curve will represent a change in the excitability of the $\alpha$ motoneuron pool (Funase et al., 1994; Komiyama, Kawai, \& Fumoto, 1999). A steeper 
slope reflects greater motoneuron excitability and a flatter slope reflects a lesser degree of motoneuron excitability.

In addition to the linear regression equation, EXCEL also gives the value of the square of the correlation coefficient $\left(\mathrm{R}^{2}\right)$ as part of the analysis. This assesses the proportion of variation in the observed data that can be explained by the linear regression equation to the total variation in the data. If there is zero unexplained variation, $\mathrm{R}^{2}$ will be 1 . Thus, $\mathrm{R}^{2}$ values close to 1 will indicate higher linearity of the data points chosen.

The ascending slope of the M-wave recruitment curve also showed a linear relationship between M-wave amplitudes and stimulus intensity, reflecting the increase in muscle fibres responding to increase in current intensity applied to the motor nerve. Linear regression analysis was applied in the same manner to this portion of the M-wave recruitment curve. The slope of the line described the increase in M-wave amplitude, and, indirectly, the number of muscle fibres recruited per unit increase in stimulus intensity. This was called M-slope $\left(\mathrm{M}_{\mathrm{slp}}\right)$.

\subsubsection{Calculation of $\mathrm{H}$-slope $\left(\mathrm{H}_{\text {slp }}\right)$}

Four methods had been used to select the data points on the ascending slope of the Hreflex recruitment curve for linear regression analysis. In the first method all the data measured at stimulus intensities less than the threshold of the $M$-wave $\left(M_{t h}\right)$ were included (Funase et al., 1994). In the second method the mean slope of the Hrecruitment curve up to the half-maximal response size was estimated (Hilgevoord et al., 1994). In the third method the data between $\mathrm{H}_{\text {th }}$ and $\mathrm{H}_{\max }$ which produced the highest correlation coefficient $\left(\mathrm{R}^{2}\right)$ were used for plotting the linear regression line 
(Funase et al., 1996). The fourth method was introduced by Komiyama et al. (1999). Using this method, data for analysis were only taken from within the $10 \%$ to $85 \%$ of the rising part of the H-reflex recruitment curve. The reasoning was that H-reflexes taken at or near $\mathrm{H}_{\text {th }}$ are inherently unstable (Crone et al., 1990), and those closer to $\mathrm{H}_{\max }$ are likely to be contaminated by collision effect from antidromic conduction along the motor axons. Therefore, data collected from between $10 \%$ to $85 \%$ of the rising portion of the H-reflex recruitment curve provided a more reliable indication of the activity of the motoneuron pool.

When all data collected at stimulus intensities less than the threshold of the M-response $\left(\mathrm{M}_{\mathrm{th}}\right)$ are included for calculation of the linear regression equation of the H-recruitment curve (Funase et al., 1994), the $\mathrm{H}_{\text {slp }}$ represents an average rate of $\alpha$-motoneuron recruitment in response to the increase in stimulus intensity from $H_{\text {th }}$ to $M_{\text {th }}$. It will reflect the response of a significant proportion of the pool of motoneurons involved. However, $\alpha$-motoneuron excitability can be unstable around threshold level of $\mathrm{H}$ reflexes (Crone et al., 1990). The incorporation of data from this part of the curve will make the resultant $\mathrm{H}_{\text {slp }}$ less sensitive as a tool for comparing the before and after effects of an intervention on the motoneuron pool excitability.

When only the mean slope of the H-recruitment curve up to the half-maximal response size is estimated, the collision effects from antidromic conduction are eliminated (Hilgevoord et al., 1994). However, because the motoneurons contributing to the ascending slope of the H-reflex recruitment curve are recruited in order, from small to large cell sizes, according to the "size principle" (Henneman, Somjen, \& Carpenter, 1965a; Henneman, Somjen, \& Carpenter, 1965b), the $\mathrm{H}_{\text {slp }}$ as calculated will probably 
only reflect changes in the excitability of the smaller or intermediate sized $\alpha$ motoneurons which only constitute a portion of the motoneuron pool. On the other hand, this part of the slope on the H-recruitment curve is generally more linear then the rest and will probably help to detect smaller changes in the H-slopes.

When the data between $\mathrm{H}_{\text {th }}$ and $\mathrm{H}_{\text {max }}$ producing the highest correlation coefficient $\left(\mathrm{R}^{2}\right)$ are used for plotting the linear regression line (Funase et al., 1996), it ensures that the data points used are along the most linear part of the linear regression line. However, it is not always possible that the $H_{\text {slp }}$ with the highest correlation coefficient $\left(R^{2}\right)$ in all trials in the same experiment fall into the same range of stimulus intensities so that sensible comparison can be made.

This present study adopted an approach similar to Funase's first method in that all data measured at stimulus intensities less than the threshold of the M-response $\left(\mathrm{M}_{\mathrm{th}}\right)$ and on the rising part of the recruitment curve were included (Fig.2.3). In general, the data points chosen for fitting the linear regression equations were above $\mathrm{H}_{\text {th }}$ but below $\mathrm{H}_{\max }$, so that the unstable and contaminated parts of the recruitment curve were excluded (Crone et al., 1990; Funase et al., 1996). This was facilitated by selecting the first stimulus intensity after $\mathrm{H}_{\text {th }}$ to be $\left[\mathrm{H}_{\text {th }}+15 \%\left(\mathrm{H}_{\max }-\mathrm{H}_{\text {th }}\right)\right]$, which puts it significantly above $\mathrm{H}_{\text {th }}$. The same range of stimulus intensities was used for determining the $\mathrm{H}_{\text {slp }}$ across the trials within the same experiment. This ensured comparison of the $\alpha$ motoneuron excitability over the same range of stimulus intensities for the particular experiment. 


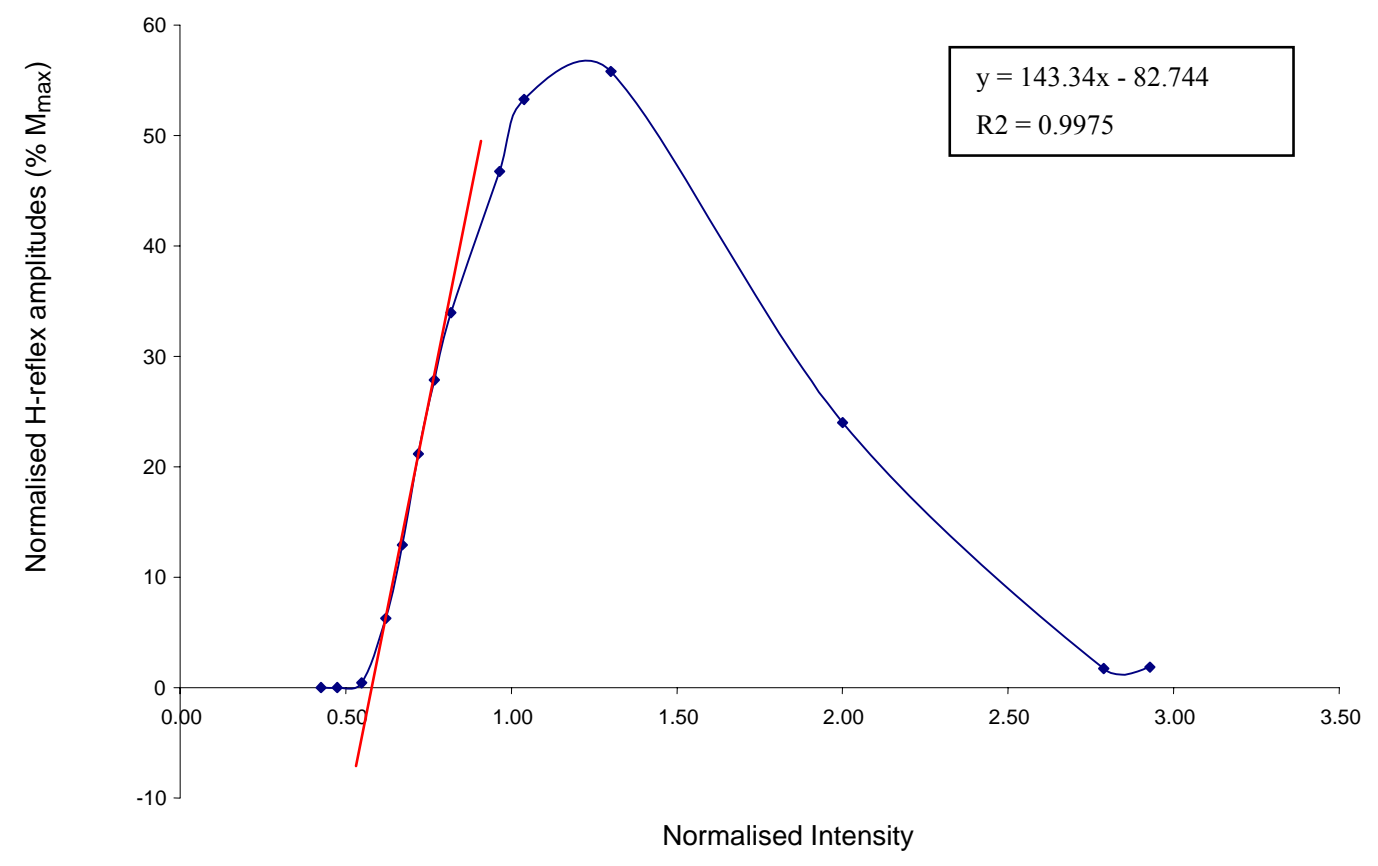

Fig. 2.3 Linear regression analysis of the ascending slope of the H-reflex recruitment curve. A linear regression line (in red) was fitted to data points on the linear ascending portion of the H-reflex recruitment curve. $\mathrm{M}_{\mathrm{th}}$ is represented as 1.00 on the $x$-axis. In this example, the linear regression analysis was performed using EXCEL. The linear regression equation in the textbox gave an $\mathrm{H}$-slope of 143.34 . The square of the correlation coefficient $\left(R^{2}\right)$ was 0.9975 , and indicated that the chosen data points had high linearity. 


\subsubsection{Calculation of M-slope ( $\left.\mathbf{M}_{\text {slp }}\right)$}

$\mathrm{M}_{\text {slp }}$ was calculated from data points in the linear ascending portion of the M-wave recruitment curve, using the linear regression equation as follows:

$\mathbf{Y}=\mathbf{A}+\mathbf{B X}$

Where $\quad B=$ Slope of the line

A = Constant (M-wave amplitude when stimulus intensity is 0 )

Y = Averaged M-Wave Amplitude

$\mathbf{X}=$ Stimulus Intensity

The slope of the linear part of the curve depicts the increase in number of recruited motor fibres in the peripheral nerve at the stimulus site with increasing intensity of stimulation relative to $\mathrm{M}_{\mathrm{th}}$ (Funase et al., 1996). An example of linear regression analysis of the slope of an M-wave recruitment curve is shown in Fig. 2.4. 


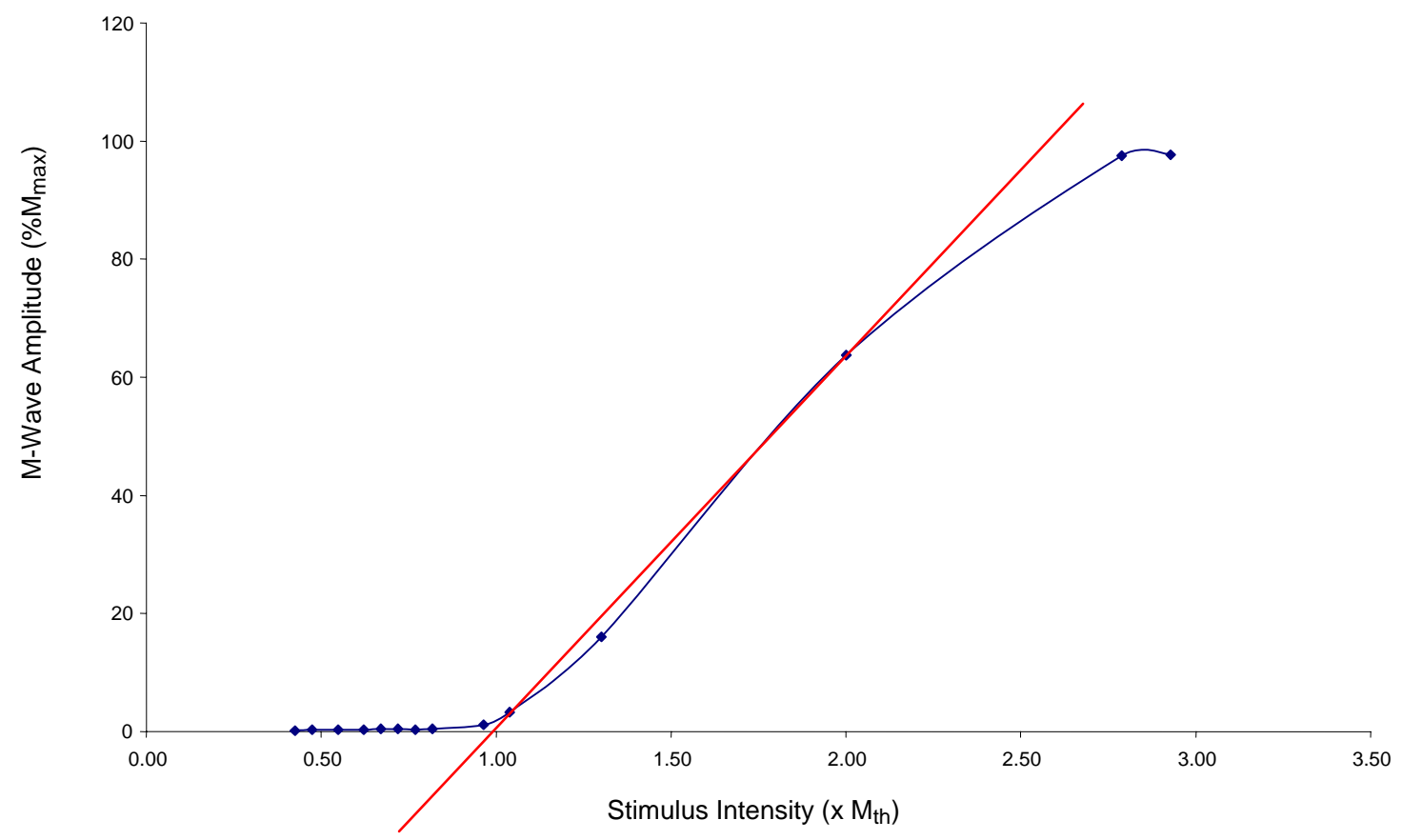

Fig. 2.4 Linear regression analysis of the ascending slope of an M-wave recruitment curve. The data points used are those situated on the linear ascending portion of the M-wave recruitment curve between $M_{t h}$ and $M_{\max }$. The graph shows a linear regression line (in red) fitted to this part of the curve. The slope was 61.646 and correlation coefficient $\left(R^{2}\right)$ was 0.9927 . 


\subsection{Analysis of M-wave Stability}

In this study, changes in $\alpha$-motoneuron excitability were determined by changes in the H-slope. The H-slope was calculated by fitting a linear regression line to the ascending slope of the H-reflex recruitment curve. However, changes in peripheral conditions could lead to changes in H-reflexes (Davies \& Lader, 1983; Delwaide, 1977; Ishikawa, Ott, Porter, \& al, 1966; Kerr, Vujnovich, \& Bradnam, 2002a). When a mixed peripheral nerve is stimulated, different phases of motor fibres responses are reflected in the shape of the sigmoidal M-wave recruitment curve. If it is assumed that the scatter of electrical excitability in the population axons contained in a peripheral nerve follows a standard normal Gaussian distribution, then the M-wave recruitment curve should be reproducible. Thus when M-wave recruitment curves recorded in separate experiments on a given subject are identical, the technical conditions for stimulation and recording can be assumed to be fairly similar (Hugon, 1973). Indeed, M-wave recruitment curves have been shown to be reproducible in the same subject at rest (Crayton \& King, 1981; Funase et al., 1994; Williams, Sullivan, Seaborne, \& Morelli, 1992). However, Mwaves have also been shown to be altered by changes in positioning of recording electrodes, from unrestrained ankle of the recording leg (Little et al., 1989) and from muscle contraction (Funase et al., 1994).

Four methods have been used to assess M-wave stability in the scientific literature to date, namely the nomination of a percent change in M-wave amplitude (Kukulka et al., 1986; Morelli et al., 1998; Paquet \& Hui-Chan, 1999), superimposition of the Mrecruitment curves (Murphy et al., 1995), statistical analysis of variation of the M-wave amplitude across conditions (Allison \& Abraham, 1995; Dishman \& Bulbulian, 2001; 
Goldberg, Seaborne, Sullivan, \& Leduc, 1994; Morelli, Chapman, \& Sullivan, 1999), and the M-slope intercept method (Kerr et al., 2002b).

\subsubsection{Intercept Method}

In this study, the M-slope intercept method was used to establish M-wave stability (Kerr et al., 2002b). According to Kerr, $y$-intercept of the M-slope includes components of $\mathrm{M}_{\mathrm{th}}, \mathrm{M}_{\mathrm{slp}}$, and $\mathrm{M}_{\max }$. SPSS for Windows programme (version 11.0.1, Copyright CSPSS Inc. 1989-2001) was used to calculate the upper bound and lower bound of the $95 \%$ confidence intervals of the $y$-intercepts of the linear regression lines applied to the rising section of each of the M-wave recruitment curves. There is a $95 \%$ probability that the true value of the $y$-intercept will lie within this interval. The $95 \%$ confidence intervals of the $y$-intercepts across the trials within the same experiment were then compared. If the $95 \%$ confidence intervals of all the $y$-intercepts overlapped, then the M-waves were considered to be stable. This set of data would then be accepted into the pooled data for final analysis. Data from an experiment in which the $95 \%$ confidence intervals of one or more of the M-wave $y$-intercepts did not overlap with the rest would be excluded.

During the process of calculation, it was found that using the averaged M-wave amplitudes resulted in very wide $y$-intercept confidence intervals of doubtful significance even though they overlapped. An example is shown using the figures in Table 2.2. In this example the slope of the linear regression line using SPSS was 61.565. The lower bound of the 95\% Confidence Interval was -83.521 and the upper bound was -37.521 . The width of the interval was 46. Using EXCEL, the slope of the linear regression line calculated was slightly different ( lope $\left.=61.646, \mathrm{R}^{2}=0.9927\right)$. 
Table 2.2 Normalised Averaged M-wave Amplitudes versus Normalised Stimulation Intensities

\begin{tabular}{|l|l|}
\hline $\begin{array}{l}\text { Normalised } \\
\text { Intensity }\end{array}$ & $\begin{array}{l}\text { Normalised } \\
\text { Averaged } \\
\text { Amplitudes }\end{array}$ \\
\hline 0.96 & 1.136041 \\
\hline 1.04 & 3.266118 \\
\hline 1.30 & 16.04658 \\
\hline 2.00 & 63.7603 \\
\hline
\end{tabular}

Table 2.3 Linear Regression Analysis of averaged M-wave amplitudes in Table 2.2 using SPSS

Coefficients $^{\mathrm{a}}$

\begin{tabular}{|c|c|c|c|c|c|c|c|c|}
\hline \multirow[b]{2}{*}{ Model } & & \multicolumn{2}{|c|}{$\begin{array}{l}\text { Unstandardized } \\
\text { Coefficients }\end{array}$} & \multirow{2}{*}{$\begin{array}{c}\text { Standardized } \\
\text { Coefficients } \\
\text { Beta }\end{array}$} & \multirow[b]{2}{*}{$\mathrm{t}$} & \multirow[b]{2}{*}{ Sig. } & \multicolumn{2}{|c|}{ 95\% Confidence Interval for B } \\
\hline & & $\mathrm{B}$ & Std. Error & & & & Lower Bound & Upper Bound \\
\hline 1 & (Constant) & -60.521 & 5.345 & & -11.322 & .008 & -83.521 & -37.521 \\
\hline & $\begin{array}{l}\text { Normalised } \\
\text { Stimulation Intensity }\end{array}$ & 61.565 & 3.854 & .996 & 15.972 & .004 & 44.980 & 78.149 \\
\hline
\end{tabular}

a. Dependent Variable: Normalised Averaged Amplitudes 
In order to make the calculation more precise, all the normalised M-wave amplitudes before averaging and within the same range of stimulus intensities as in the previous analysis were used for calculating the $95 \%$ confidence intervals of the $y$-intercept (Table 2.4). It can be seen that the $95 \%$ confidence interval was now much narrower with a lower bound of -66.090 and an upper bound of -55.339 (Table 2.5). The width of the interval was narrowed to 10.75 from the previous figure of 46 , which was calculated from averaged amplitudes. A narrower width of the confidence interval helps to avoid accepting data which should have been excluded.

It is also interesting to note that the slope of the linear regression line was now 61.646, exactly the same as that calculated from the averaged amplitudes by using EXCEL (Microsoft ® Excel 2002 SP-1, Copyright (C) Microsoft Corporation, 19852001). Therefore, the use of pre-averaged amplitudes for calculation of confidence intervals allowed more precise comparison of the M-slope. The gradients of the slopes calculated by SPSS using this method were also consistent with those calculated by EXCEL from the averaged amplitudes. The data on H-slope and M-slope for final analysis in this study were based on calculations using EXCEL. 
Table 2.4 Normalised Pre-averaged M-wave Amplitudes verus Normalised Stimulation Intensities.

\begin{tabular}{|l|l|}
\hline $\begin{array}{l}\text { Normalised } \\
\text { Intensity }\end{array}$ & $\begin{array}{l}\text { Normalised } \\
\text { Amplitudes }\end{array}$ \\
\hline 0.96 & 0.85 \\
\hline 0.96 & 1.7 \\
\hline 0.96 & 0.85 \\
\hline 1.04 & 2.98 \\
\hline 1.04 & 2.98 \\
\hline 1.04 & 3.83 \\
\hline 1.3 & 15.76 \\
\hline 1.3 & 16.19 \\
\hline 1.3 & 16.19 \\
\hline 2 & 63.48 \\
\hline 2 & 65.18 \\
\hline 2 & 62.62 \\
\hline
\end{tabular}

Table 2.5 Linear Regression Analysis of pre-averaged M-wave amplitudes in Table 2.4 using SPSS

Coefficients $^{\mathrm{a}}$

\begin{tabular}{|c|c|c|c|c|c|c|c|c|}
\hline \multirow[b]{2}{*}{ Model } & & \multicolumn{2}{|c|}{$\begin{array}{l}\text { Unstandardized } \\
\text { Coefficients }\end{array}$} & \multirow{2}{*}{$\begin{array}{c}\begin{array}{c}\text { Standardized } \\
\text { Coefficients }\end{array} \\
\text { Beta }\end{array}$} & \multirow[b]{2}{*}{$\mathrm{t}$} & \multirow[b]{2}{*}{ Sig. } & \multicolumn{2}{|c|}{$95 \%$ Confidence Interval for B } \\
\hline & & $\mathrm{B}$ & Std. Error & & & & Lower Bound & Upper Bound \\
\hline & (Constant) & -60.710 & 2.410 & & -25.187 & .000 & -66.080 & -55.339 \\
\hline & NINT & 61.646 & 1.737 & .996 & 35.498 & .000 & 57.776 & 65.515 \\
\hline
\end{tabular}

a. Dependent Variable: NM1 


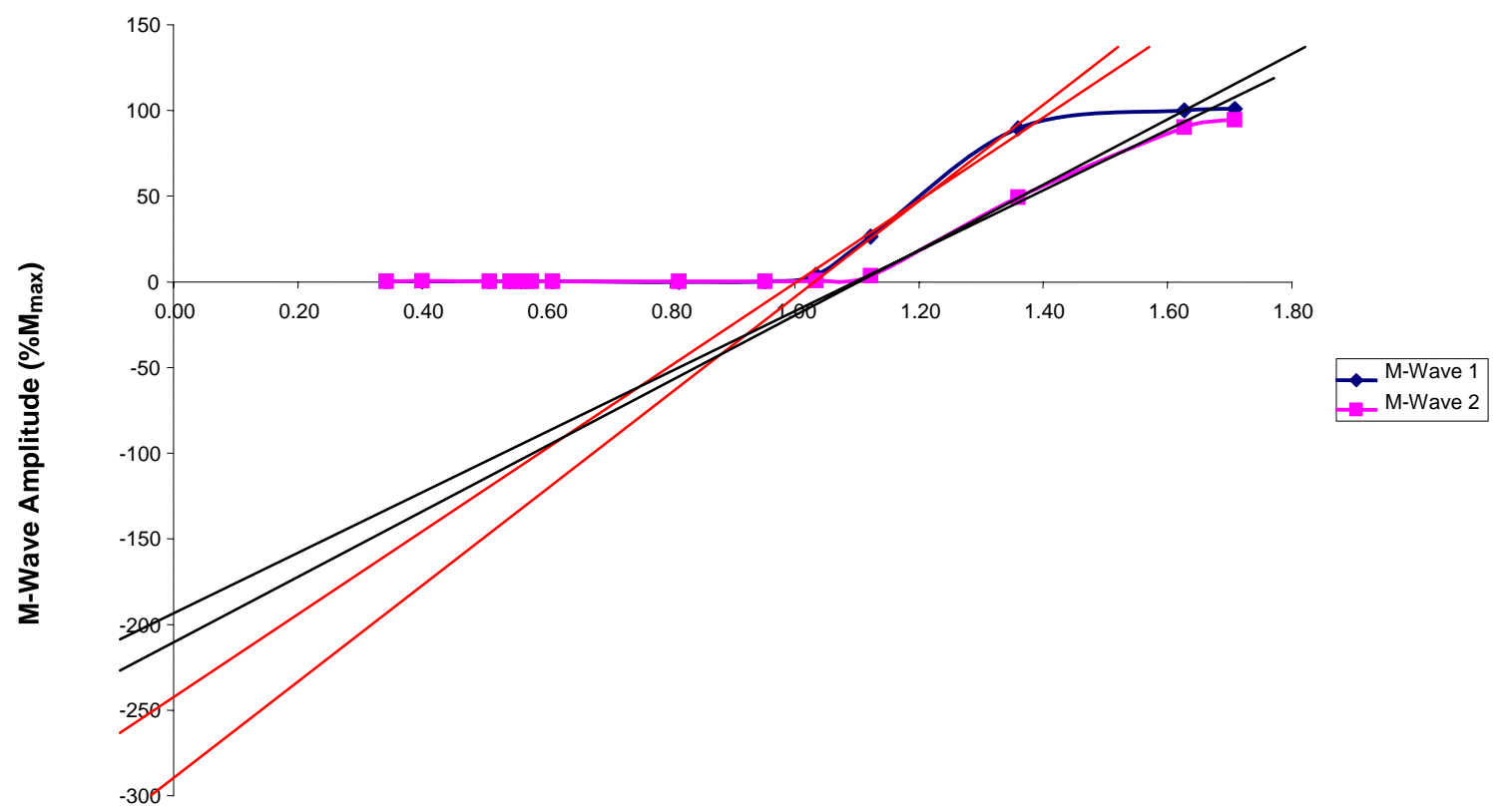

Stimulus Intensity $\left(x \mathrm{M}_{\mathrm{th}}\right)$

Fig. 2.5 Examination of M-wave stability using the Intercept method. M-wave recruitment curves 1 and 2 (in black and pink respectively) with linear regression lines (in red and black respectively) showing the upper and lower bound of the 95\% confidence intervals of their $y$ intercepts. In this case, the 95\% confidence intervals of the $y$-intercepts did not overlap, indicating unstable M-waves and thus instability of either the peripheral recording or stimulating conditions. The data from this experiment were excluded from final analysis. 


\subsubsection{Exceptions to Intercept Method}

When using the intercept method to assess M-wave stability in this study, data from 3 subjects were originally excluded from final analysis because the $95 \%$ confidence intervals of the M-slopes linear regression lines did not overlap. However, on examination of the original $\mathrm{M}$-wave recruitment curves, they were found to be very closely approximated and almost overlapped. It was, therefore, unreasonable to exclude the data from those experiments from analysis. A decision was then made to modify the criteria for acceptance of data for final analysis. It was decided that if the difference of the lower and upper bound $95 \%$ confidence intervals of a pair of non-overlapping $y$ intercepts was less than $1 \%$ of the mean of the two values, the set of data would still be accepted. 


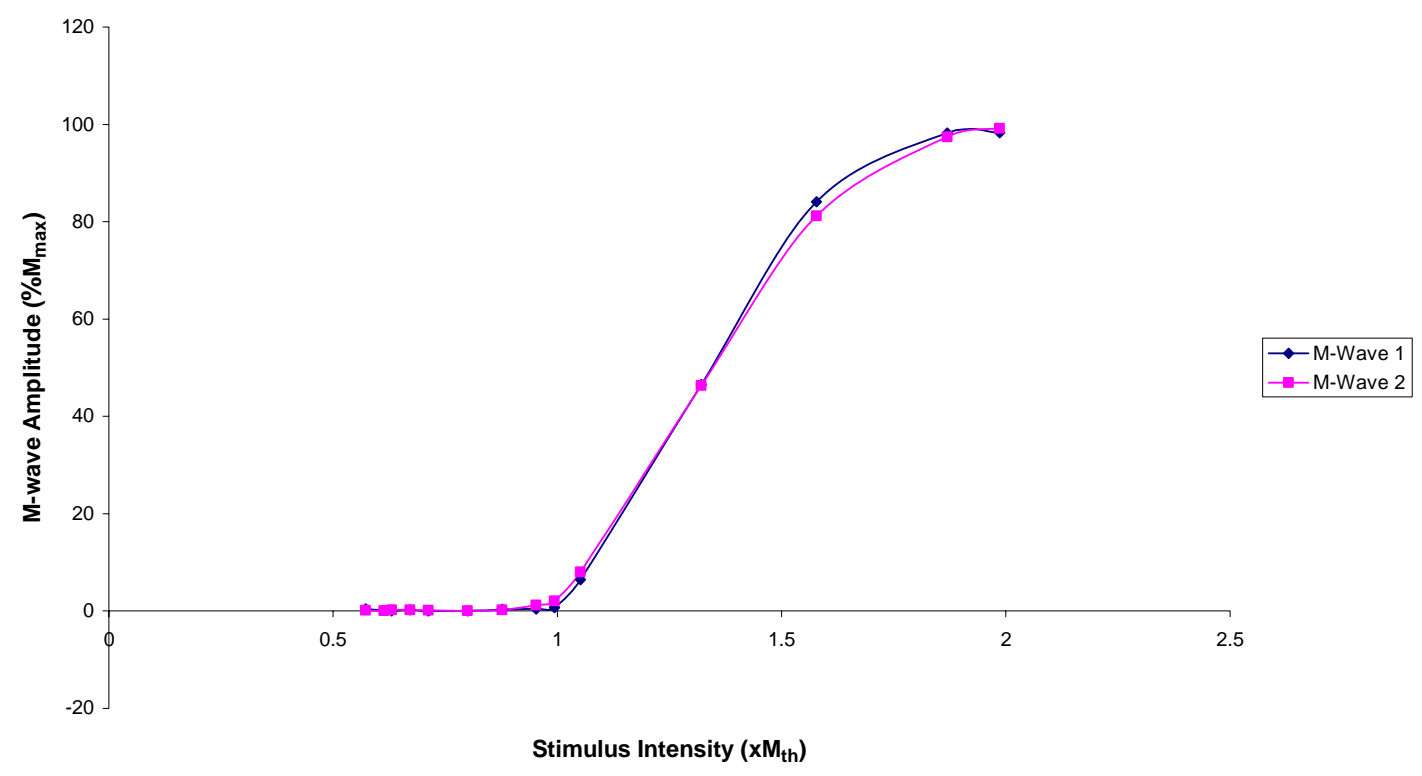

Fig. 2.6 Overlapped M-wave recruitment curves with non-overlapping $y$-intercept confidence intervals. The $95 \%$ confidence intervals of the linear regression lines applied to the steep rising slopes of the curves were $(-155.01,-142.52)$ and $(-142.35,-133.37)$ respectively. The mean of the lower bound 95\% confidence interval of the first line and the upper bound 95\% confidence interval of the second line was $(-142.52-142.35) / 2=-142.44$. Since -142.52 and -142.35 fell within one percent on either side of -142.44 (-143.86, -141.02), the M-waves were still considered to be stable and data from the experiment were accepted for final analysis. 


\subsubsection{Reliability Test using Intraclass Comparison}

While analysis of variance had been used in the past for assessing M-wave stability (Allison \& Abraham, 1995; Morelli et al., 1999), it was shown to be an insensitive test for this purpose (Kerr et al., 2002a, 2002b). To screen and confirm the consistency of the M-slopes across the trials, intraclass correlation coefficient (ICC) was calculated using SPSS for Windows (version 11.0.1, Copyright CSPSS Inc. 1989-2001). While analysis of variance assesses significance in differences, intraclass correlation examines the degree of similarity or repeatability of measurements. Intraclass correlation is sensitive to any systematic change in the means of the scores (Shrout \& Fleiss, 1979) and thus can be used to give an overall index of repeatability and stability of the Mslopes across the trials. During the preliminary analysis, it was found that an ICC of 0.8960 was not sensitive enough to exclude unstable M-waves. Therefore, a higher ICC of 0.95 or above was chosen as the criteria for screening stability or repeatability of the M-slopes across the trials and of the control H-slopes in Trials 1 and 2.

\subsubsection{Analysis of Baseline Stability of the H-reflex.}

Baseline stability of the H-reflex was also established by intraclass correlation analysis of the recruitment curves in Trials 1 and 2 .

\subsection{Assessment of Motoneuron Excitability by $\mathrm{H}_{\text {slp }}$ and $\mathrm{H}_{\text {slp }} / \mathbf{M}_{\text {slp }}$}

$\mathrm{H}_{\text {slp }}$ and $\mathrm{H}_{\text {slp }} / \mathrm{M}_{\text {slp }}$ data for all participants were pooled according to trials. The means and standard deviations of $\mathrm{H}_{\text {slp }}$ and $\mathrm{H}_{\mathrm{slp}} / \mathrm{M}_{\text {slp }}$ from each trial were calculated. Control trials $\mathrm{H}_{\text {slp }}$ and $\mathrm{H}_{\text {slp }} / \mathrm{M}_{\text {slp }}$ data were pooled and averaged. The difference between the $\mathrm{H}_{\text {slp }}$ and $\mathrm{H}_{\mathrm{slp}} / \mathrm{M}_{\text {slp }}$ in the post-acupuncture trials (Trials 3, 4 or 5) and the control trial mean 
(from Trials 1 and 2) for each stimulus intensity was designated a change score and expressed as a percentage of the control trial mean by the following formula:

Percentage Change Score $=\frac{\text { Trial Mean }(3,4 \text { or 5) }- \text { Control Trial Mean }}{\text { Control Trial Mean }} \times 100$

The percentage change score allows assessment of percentage change from baseline control trial mean and also comparison of the effect of time at different intervals after acupuncture as well as the effect of different durations of acupuncture.

\subsection{Assessment of Intensity of Acupuncture Sensation (deqi)}

Intensity of acupuncture sensation (deqi) was assessed by using a $100 \mathrm{~mm}$ horizontal Visual Analogue Scale, divided into 10 equal sections. Zero (0) on the extreme left represented no acupuncture sensation, and 10 on the extreme right represented the strongest imaginable acupuncture sensation. The subjects were instructed to grade and report any aching, pulling, heavy, dull, numb, or throbbing sensation after introduction of the needles. This sensation was maintained, with or without manipulation of the needle, over one minute for every 5 minutes of acupuncture. The subjects were asked to average out and grade the intensity of the sensation afterwards as it could vary during the minute of observation.

\subsection{Statistical Analysis}

Statistical analysis was performed using SPSS for Windows software programme. For all statistical tests, the level of significance was set at $p<0.05$. For repeated measures 
ANOVA, when the assumption of sphericity was not violated, results from analysis assuming sphericity were used because this method of analysis is more sensitive for smaller sample sizes. When the assumption of sphericity was violated, results from multivariate analysis were used. 


\section{Chapter $3 \quad$ Results}

\subsection{Introduction}

Twenty (20) subjects participated in the study. There were 8 males and 12 females with age between 24 to 56 years $($ mean $=36.7)$. Fourteen $(14)$ participants had previous experience with acupuncture.

All subjects received short duration and sustained acupuncture on two separate occasions in a random fashion. Changes in the $\alpha$-motoneuron pool excitability were assessed by using the H-reflex technique. Data were grouped according to the duration of acupuncture and time of observation. The effects of short duration and sustained acupuncture on the $\alpha$-motoneuron pool excitability were analysed separately, and then compared. Correlation analysis of intensity of acupuncture sensation (deqi) to changes in $\alpha$-motoneuron pool excitability was also performed.

\subsection{Effects of Short Duration Acupuncture}

To determine the effects of short duration acupuncture on $\alpha$-motoneuron excitability, the subjects received manual acupuncture for 5 minutes. Timing began when the subject reported the appearance of acupuncture sensation (deqi). H-reflexes were collected before, immediately following and then 15 minutes after the withdrawal of the acupuncture needles. 


\subsubsection{M-slope stability}

\subsubsection{Analysis of M-slope Stability in Trials 1-4 (Preliminary)}

In order to attribute acupuncture effects to changes in the central nervous system, it was important to demonstrate stability of peripheral conditions during the experiments. An M-slope was calculated by fitting a linear regression line to the ascending slope of the M-wave recruitment curve from the same trial. The M-slope provided an objective measure of the stability of the stimulating and recording conditions (Allison \& Abraham, 1995; Bradnam et al., 2000; Kerr et al., 2002b). M-slopes from different trials were, therefore, examined to establish stability of peripheral conditions throughout the experiments.

M-slope data from all subjects in Trials 1-4 are shown in Fig. 3.1A. In some subjects, a marked fluctuation in the M-slope across the four trials indicated unstable peripheral conditions which might affect the H-reflex. In these instances, changes in the H-reflex could not be totally ascribed to changes in the central nervous system. Therefore, data from these experiments had to be excluded from final analysis.

The single intraclass correlation coefficient (ICC) of the M-slopes from all subjects across Trials $1-4$ was 0.8960 . This was below the criteria $(\mathrm{ICC}=0.95)$ set for stability (Refer section 2.6.3). Therefore, the M-slopes from all trials were subjected to further analysis.

\subsubsection{Analysis of M-slope Stability in Trials 1-4 (Intercept Method)}

Six (6) subjects had non-overlapping 95\% confidence intervals when examined using the Intercept Method (Kerr et al., 2002b). The results of the intercept confidence intervals analysis are presented in Appendix V. The ICC became 0.9752 after excluding 
data from these 6 subjects. However, in two of these subjects, the closest upper bound and lower bound of the $95 \%$ confidence intervals were within $1 \%$ of the mean between them. The M-wave recruitment curves also appeared to overlap visually in these subjects. For these reasons, their data were accepted for final analysis. Data from the four subjects with wider gaps in the $95 \%$ confidence intervals of the $y$-intercepts were, however, excluded.

Intraclass correlation analysis of M-slopes from Trials 1-4 was repeated for the remaining 16 subjects. The ICC was found to have improved from the original 0.8960 to 0.9740, which was above the criteria set for stability. Repeated measures ANOVA of M-slopes from Trials 1-4 also demonstrated no significant difference between them ( $p$ $=0.974)$. There is now much less within-subject M-slope variation among the remaining subjects (Fig. 3.1 B). Results of the tests reflected highly consistent M-slopes from Trials 1-4 and indirectly confirmed stable peripheral conditions during the experiments in the 16 subjects whose data were included for final analysis in the short duration acupuncture group. Changes in the $\alpha$-motoneuron excitability could then be ascribed to a change in central nervous system excitability. 


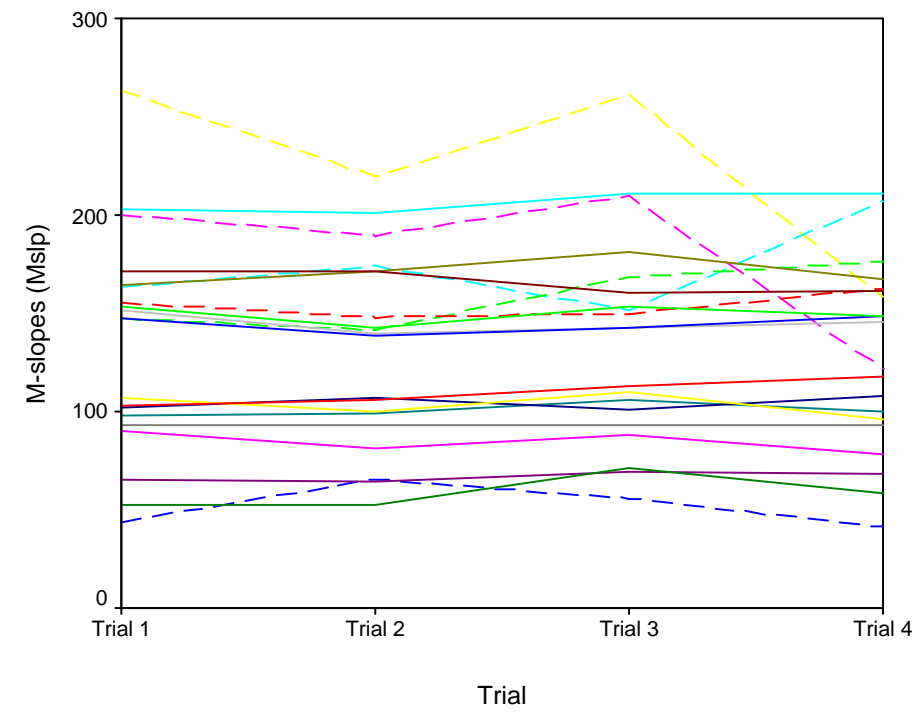

Fig. 3.1A Changes in $M_{s / p}$ in Trials 1-4 in short duration acupuncture $(n=20)$. Individual subject's M-slopes from the four trials are shown. Marked fluctuation in M-slopes during the experiment in some subjects is demonstrated.

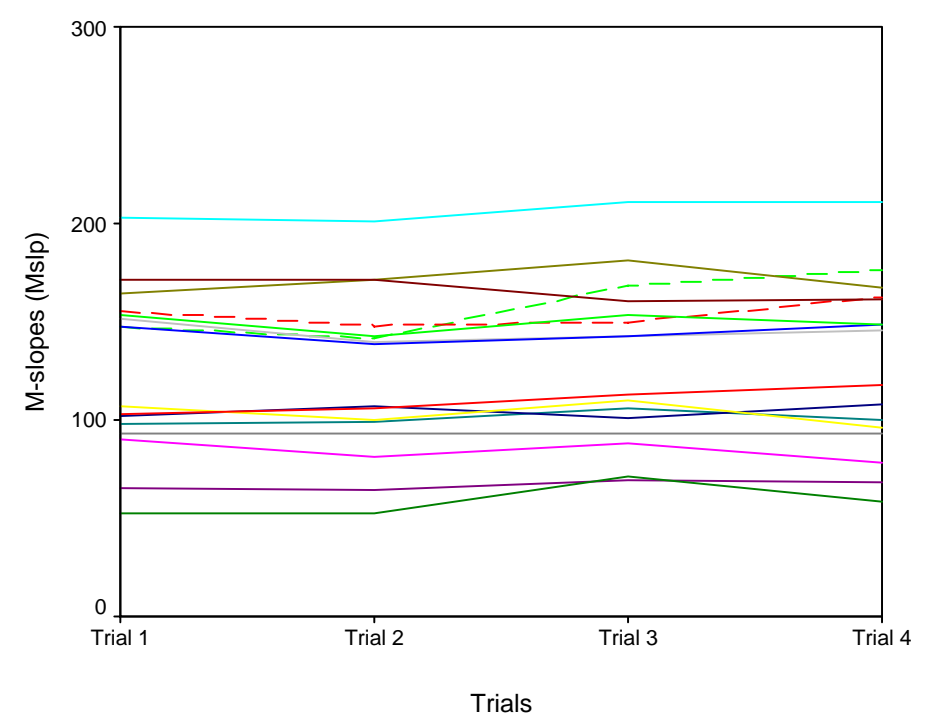

Fig. 3.1B Changes in $M_{\text {sip }}$ in Trials 1-4 in short duration acupuncture $(n=16)$. Data from 4 subjects with non-overlapping $y$-intercepts 95\% confidence intervals were excluded. Two (2) subjects with non-overlapping $y$-intercepts $95 \%$ confidence intervals were included under special conditions. Only small fluctuations in M-slopes from Trials 1-4 are now apparent. 


\subsubsection{Analysis of $\mathbf{H}$-slopes Stability in Trials 1-2}

In this study two H-reflex recruitment curves were recorded before acupuncture to test the stability of the $\alpha$-motoneuron pool. The slopes of these two H-reflex recruitment curves were then assessed for stability and repeatability. A stable baseline was important for comparison to H-slopes collected post-acupuncture so that the effect of acupuncture on $\alpha$-motoneuron excitability could be measured.

The ICC of the two H-slopes in Trials $1-2$ of the 16 subjects was 0.9784 . The means of the H-slopes in Trials 1 and 2 were also compared by using Paired-Sample T-Test. There was no significant difference between the H-slopes of Trials 1 and $2(p=0.981)$. Both tests indicated a high degree of consistency and stability of the $\alpha$-motoneuron pool prior to acupuncture.

\subsubsection{Changes in Motoneuron Excitability}

Both H-slope $\left(\mathrm{H}_{\mathrm{slp}}\right)$ and $\mathrm{H}$-slope/M-slope ratio $\left(\mathrm{H}_{\mathrm{slp}} / \mathrm{M}_{\mathrm{slp}}\right)$ have been used as parameters for assessing changes in $\alpha$-motoneuron excitability in the H-reflex methodology (Bradnam et al., 2000; Funase et al., 1994). However, $\mathrm{H}_{\text {slp }}$ and $\mathrm{H}_{\mathrm{slp}} / \mathrm{M}_{\text {slp }}$ have also been found to have different sensitivities during different experimental interventions (Bradnam et al., 2000; Funase et al., 1994). In this study, changes in motoneuron excitability were, therefore, assessed by comparing both $\mathrm{H}_{\mathrm{slp}}$ and $\mathrm{H}_{\mathrm{slp}} / \mathrm{M}_{\mathrm{slp}}$ before and after acupuncture.

\subsubsection{Changes in $\mathrm{H}_{\text {slp }} \underline{\text { after short acupuncture }}$}

$\mathrm{H}_{\text {slp }}$ estimates the recruitment properties of an $\alpha$-motoneuron pool (Funase et al., 1994). An increase in $\mathrm{H}_{\text {slp }}$ indicates an increase in $\alpha$-motoneuron pool excitability while a 
decrease in $\mathrm{H}_{\text {slp }}$ indicates a reduction. The mean of the $\mathrm{H}_{\text {slp }}$ in Trials 1 and 2 was calculated and referred to as baseline. Individual changes in $\mathrm{H}_{\text {slp }}$ during the experiment are shown in Fig. 3.2. The means and standard deviations of $\mathrm{H}_{\text {slp }}$ taken during the experiment were calculated and shown in Table 3.1. The large standard deviations reflected the marked inter-individual differences in baseline $\mathrm{H}_{\mathrm{slp}}$ and individual subject's responses to acupuncture.

To determine significant differences in $\mathrm{H}_{\text {slp }}$ between baseline and Trials $3-4, \mathrm{H}_{\text {slp }}$ from individual subjects were pooled and analysed by repeated measures ANOVA. There was no significant difference in $\mathrm{H}_{\text {slp }}$ between the baseline and Trials 3 or $4(p=0.484)$. Between-subjects effect, however, was found to be significant $(p<0.0005)$. The results indicated that short duration acupuncture had no significant effects on $\alpha$-motoneuron excitability as assessed by comparison of $\mathrm{H}_{\text {slp }}$. On the other hand, a wide variation in individual responses to acupuncture was demonstrated. 


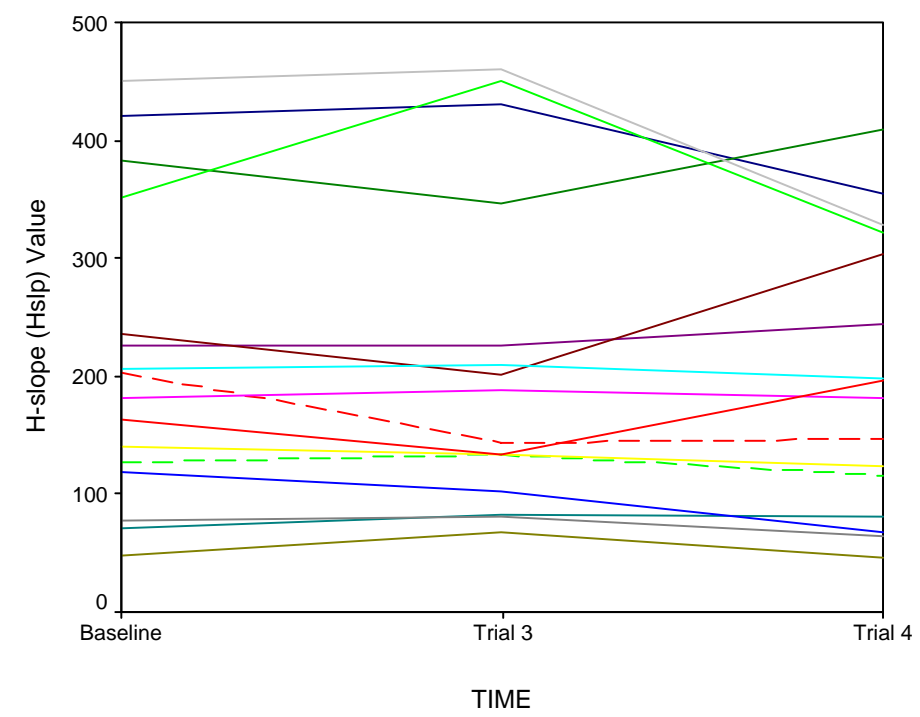

Fig. 3.2 Changes in $\mathrm{H}_{\text {slp }}$ (baseline, Trials 3-4) in short duration acupuncture $(\mathrm{n}=16)$. Marked inter-individual variability in baseline $\mathrm{H}_{\mathrm{slp}}$ and response to acupuncture at trial 3 and 4 in different subjects is demonstrated.

Table 3.1 Descriptive statistics of $\mathrm{H}_{\text {slp }}$ (baseline, trials 3-4) in short duration acupuncture.

Descriptive Statistics

\begin{tabular}{|l|r|r|r|r|r|}
\hline & \multicolumn{1}{|c|}{$\mathrm{N}$} & Minimum & Maximum & \multicolumn{1}{c|}{ Mean } & Std. Deviation \\
\hline $\mathrm{H}_{\text {sIp }}$ Baseline & 16 & 47.60 & 449.90 & 212.6357 & 126.26296 \\
$\mathrm{H}_{\text {sIp }}$ trial 3 & 16 & 66.97 & 460.16 & 211.9068 & 135.54142 \\
$\mathrm{H}_{\text {sip }}$ trial 4 & 16 & 46.30 & 408.78 & 198.8284 & 115.77814 \\
\hline
\end{tabular}




\subsubsection{Percentage Change in $\mathrm{H}_{\text {slp }}$ after short duration acupuncture}

Because of significant inter-individual variations in $\mathrm{H}_{\text {slp }}$ as demonstrated by the large standard deviations (Table 3.1), small to moderate changes in the $\mathrm{H}_{\text {slp }}$ might not be detected using analysis of variance. Representation of the change in $\mathrm{H}_{\text {slp }}$ from baseline as percentage change is more sensitive and, therefore, may better reflect individual changes. The percentage change in $\mathrm{H}_{\text {slp }}$ was calculated as described in Methodology (Refer section 2.7).

Percentage changes in $\mathrm{H}_{\text {slp }}$ in Trials 3 and 4 after short duration acupuncture are shown in Fig. 3.3. Variations in individual responses to short duration acupuncture were apparent. The percentage changes of $\mathrm{H}_{\text {slp }}$ in Trials 3 and 4 were pooled for statistical analysis (Table 3.2). The percentages of $\mathrm{H}_{\text {slp }}$ in Trials 3 and 4 were subjected to One Sample T-test analysis. There was no significant difference between percentage changes of $\mathrm{H}_{\text {slp }}$ in Trial $3(p=0.844)$ or $4(p=0.248)$ from baseline. This indicated that there was no significant difference in $\alpha$-motoneuron excitability from baseline measured by percentage change in $\mathrm{H}_{\text {slp }}$ as a result of short duration acupuncture. The percentage changes of $\mathrm{H}_{\text {slp }}$ in Trials 3 and 4 were also compared using Paired-Sample T-test. There was no significant difference between the two trials $(p=0.31)$, which indicated the absence of significant change in motoneuron excitability between Trials 3 and 4 . 


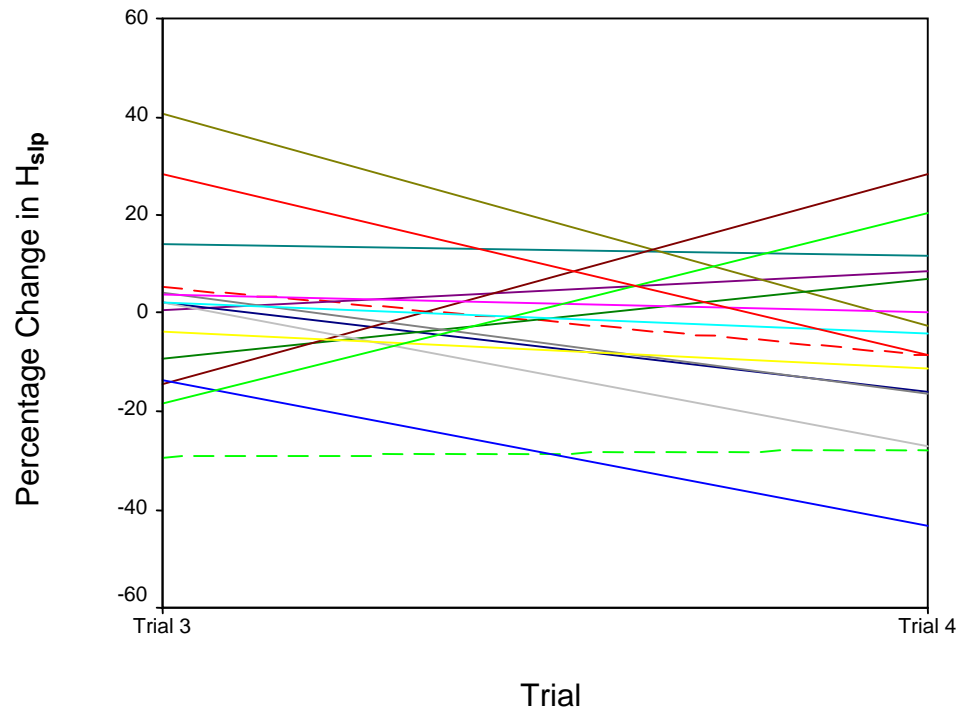

Fig. 3.3 Percentage changes in $\mathrm{H}_{\mathrm{sip}}$ in Trials 3-4 after short duration acupuncture $(n=16)$. Positive percentage change indicates increase excitability and negative percentage change indicates inhibition of the $\alpha$-motoneuron pool. Variability of individual responses is demonstrated.

Table 3.2 Descriptive statistics of percentage changes in $\mathrm{H}_{\text {sip }}$ in Trials $3-4$ after short duration acupuncture $(n=16)$.

\begin{tabular}{|l|r|r|r|r|r|}
\hline & \multicolumn{1}{|c|}{$\mathrm{N}$} & Minimum & Maximum & \multicolumn{1}{c|}{ Mean } & Std. Deviation \\
\hline $\mathrm{H}_{\text {slp }}$ \% change in Trial 3 & 16 & -29.34 & 40.67 & .8562 & 17.12623 \\
$\mathrm{H}_{\text {slp \% change in Trial 4 }}$ & 16 & -43.40 & 28.22 & -5.5869 & 18.59415 \\
\hline
\end{tabular}




\subsubsection{Change in $\mathrm{H}_{\mathrm{slp}} \mathrm{M}_{\mathrm{slp}}$ after short duration acupuncture}

The $\mathrm{H}_{\text {slp }} / \mathrm{M}_{\text {slp }}$ ratio has been used to evaluate $\alpha$-motoneuron pool excitability (Funase et al., 1994). $M_{\text {slp }}$ represents the rate of recruitment of the axons of $\alpha$-motoneurons which innervate the corresponding muscle fibres. Therefore, it can reasonably be used as a reference property of motoneurons to evaluate the reflexive $H_{\text {slp }}$ (Funase et al., 1996). An increase in $\mathrm{H}_{\mathrm{slp}} / \mathrm{M}_{\text {slp }}$ indicates an increase in $\alpha$-motoneuron pool excitability while a decrease in $\mathrm{H}_{\mathrm{slp}} / \mathrm{M}_{\mathrm{slp}}$ indicates a reduction. The mean of $\mathrm{H}_{\mathrm{slp}} / \mathrm{M}_{\mathrm{slp}}$ in Trials 1 and 2 was calculated and referred as baseline. Individual changes in $\mathrm{H}_{\mathrm{slp}} / \mathrm{M}_{\mathrm{slp}}$ during the experiment are shown in Fig. 3.4. The means and standard deviations of $\mathrm{H}_{\mathrm{slp}} / \mathrm{M}_{\mathrm{slp}}$ during the experiment were calculated and shown in Table 3.3. Inter-individual differences in baseline $\mathrm{H}_{\mathrm{slp}} / \mathrm{M}_{\mathrm{slp}}$ and responses to acupuncture were evident from the large standard deviations in comparison to the means.

To determine significant differences in $\mathrm{H}_{\text {slp }} / \mathrm{M}_{\text {slp }}$ between baseline and Trials 3-4, $\mathrm{H}_{\mathrm{slp}} / \mathrm{M}_{\text {slp }}$ from individual subjects were pooled and analysed by repeated measures ANOVA. There was no significant difference in $\mathrm{H}_{\mathrm{slp}} / \mathrm{M}_{\text {slp }}$ between the baseline and Trials 3 or $4(p=0.157)$. Between-subjects effect, however, was found to be significant $(p<0.0005)$. The results indicated that short duration acupuncture had no significant effects on $\alpha$-motoneuron excitability as assessed by comparison of $\mathrm{H}_{\mathrm{slp}} / \mathrm{M}_{\mathrm{slp}}$. On the other hand, a wide variation in individual responses to acupuncture was demonstrated. 


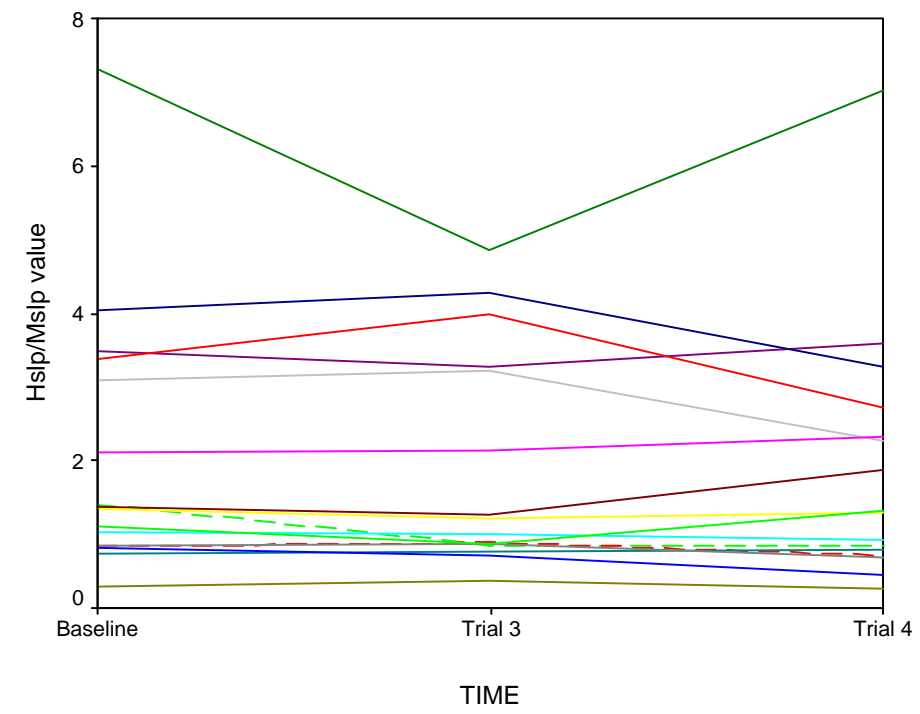

Fig. 3.4 Changes in $\mathrm{H}_{\text {slp }} / \mathrm{M}_{\text {slp }}$ (baseline, Trials 3 and 4) in short duration acupuncture $(n=16)$. Marked inter-individual variability in baseline $H_{\text {slp }} / M_{\text {slp }}$ and response to acupuncture at Trials 3 and 4 in different subjects is demonstrated.

Table 3.3 Descriptive statistics of $H_{\text {slp }} / M_{\text {slp }}$ (baseline, Trials 3 and 4) in short duration acupuncture $(n=16)$.

\section{Descriptive Statistics}

\begin{tabular}{|l|r|r|r|r|r|}
\hline & $\mathrm{N}$ & Minimum & Maximum & \multicolumn{1}{c|}{ Mean } & Std. Deviation \\
\hline Baseline $\mathrm{H}_{\text {slp }} / \mathrm{M}_{\text {slp }}$ & 16 & .28 & 7.31 & 2.0729 & 1.80854 \\
$\mathrm{H}_{\text {slp }} / \mathrm{M}_{\text {slp }}$ Trial 3 & 16 & .37 & 4.86 & 1.9114 & 1.49296 \\
$\mathrm{H}_{\text {slp }} / \mathrm{M}_{\text {slp }}$ Trial 4 & 16 & .28 & 7.01 & 1.8997 & 1.70221 \\
\hline
\end{tabular}




\subsubsection{Percentage Change in $\mathrm{H}_{\mathrm{slp}} \underline{\mathrm{M}}_{\mathrm{slp}}$ after short duration acupuncture}

Percentage changes in $\mathrm{H}_{\mathrm{slp}} / \mathrm{M}_{\mathrm{slp}}$ in Trials 3 and 4 after short duration acupuncture are shown in Fig. 3.5. Variations in individual responses to short duration acupuncture were apparent. The percentage change of $\mathrm{H}_{\mathrm{slp}} / \mathrm{M}_{\text {slp }}$ in Trials 3 and 4 were pooled for statistical analysis (Table 3.4). The percentage changes of $H_{\text {slp }} / M_{\text {slp }}$ in Trials 3 and 4 were subjected to One-Sample T-Test analysis. The percentage changes of $\mathrm{H}_{\mathrm{slp}} / \mathrm{M}_{\text {slp }}$ in Trials $3(p=0.438)$ and $4(p=0.168)$ were not significant.

This indicated there was no significant difference in $\alpha$-motoneuron excitability from baseline measured by percentage change in $\mathrm{H}_{\mathrm{slp}} / \mathrm{M}_{\mathrm{slp}}$ as a result of short duration acupuncture. The percentage change of $\mathrm{H}_{\text {slp }} / \mathrm{M}_{\text {slp }}$ in Trials 3 and 4 were also compared using Paired-Sample T-test. There was no significant difference in percentage change of $\mathrm{H}_{\text {slp }} / \mathrm{M}_{\text {slp }}$ between the two trials $(p=0.543)$, which indicated the absence of significant change in motoneuron excitability between Trials 3 and 4.

\subsubsection{Conclusion}

There was no significant change in $\alpha$-motoneuron excitability up to 15 minutes after short duration acupuncture as assessed by changes in $\mathrm{H}_{\text {slp }}$ and $\mathrm{H}_{\mathrm{slp}} / \mathrm{M}_{\text {slp }}$, or percentage changes in $\mathrm{H}_{\text {slp }}$ and $\mathrm{H}_{\text {slp }} / \mathrm{M}_{\text {slp }}$. A wide variation in individual responses to short acupuncture was observed. 


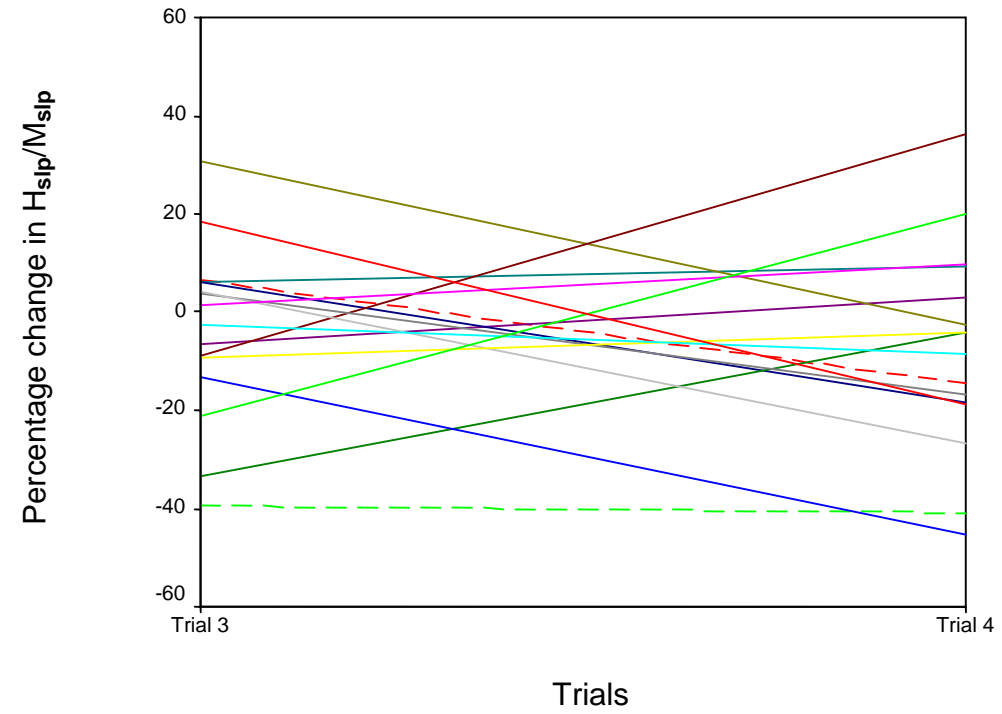

Fig. 3.5

Percentage changes in $\mathrm{H}_{\text {slp }} / \mathrm{M}_{\text {slp }}$ in Trials 3-4 after short duration acupuncture $(n=16)$.

Table 3.4 Descriptive statistics of percentage changes in $\mathrm{H}_{\mathrm{slp}} / \mathrm{M}_{\mathrm{slp}}$ in Trials 3-4 after short duration acupuncture $(n=16)$.

Descriptive Statistics

\begin{tabular}{|l|r|r|r|r|r|}
\hline & \multicolumn{1}{|c|}{$\mathrm{N}$} & \multicolumn{1}{c|}{ Minimum } & Maximum & \multicolumn{1}{c|}{ Mean } & \multicolumn{1}{c|}{ Std. Deviation } \\
\hline \% change in $\mathrm{H}_{\text {slp }} / \mathrm{M}_{\text {slp Trial 3 }}$ & 16 & -39.46 & 30.73 & -3.5425 & 17.78877 \\
$\%$ change in $\mathrm{H}_{\text {slp }} / \mathrm{M}_{\text {slp }}$ Trial 4 & 16 & -45.32 & 36.06 & -7.6625 & 21.13731 \\
\hline
\end{tabular}




\subsection{Effects of Short Duration Acupuncture (extended observation)}

To determine delayed and/or sustained effects of short duration acupuncture on $\alpha$-motoneuron excitability, 10 subjects receiving short duration acupuncture had $\mathrm{H}$ reflexes monitored up to 30 minutes after acupuncture.

\subsubsection{M-slope stability}

\subsubsection{Analysis of M-slope Stability in Trials 1-5 (Preliminary)}

M-slope data from all subjects in Trials 1-5 are shown in Fig. 3.6A. In two subjects, marked fluctuations in the M-slope within the five trials indicated unstable peripheral conditions. The ICC of the M-slopes across Trials $1-5$ was 0.8778 , which was below the criteria (ICC $=0.95)$ set for stability (Refer section 2.6.3). In view of the marked fluctuations in some subjects, the M-slopes in all trials were subjected to further analysis.

\subsubsection{Analysis of M-slope Stability in Trials 1-5 (Intercept Method)}

Three (3) subjects had non-overlapping 95\% confidence intervals when examined by the Intercept Method (Kerr et al., 2002b). However, in one of these subjects, the closest upper bound and lower bound of the $95 \%$ confidence intervals were within $1 \%$ of the mean between them. The M-wave recruitment curves also appeared to overlap visually in this subject. For these reasons, the subject's data were accepted for final analysis. Data from the two subjects with wider gaps in the $95 \%$ confidence intervals of the $y$ intercepts were excluded. The results of the intercept confidence intervals analysis are presented in Appendix V.

Intraclass correlation analysis of M-slopes from Trials 1-5 was repeated for the remaining 8 subjects. The ICC was found to have improved from the original 0.8778 to 
0.9717. Repeated measures ANOVA of M-slopes from Trials 1-5 also showed no significant difference between them $(p=0.184)$. There is now much less within-subject M-slope variation among the remaining subjects (Fig. 3.6B). Results of the tests reflected highly consistent M-slopes from Trials 1-5 and indirectly confirmed stable peripheral conditions during the experiments in the 8 subjects whose data were included for final analysis. Changes in the $\alpha$-motoneuron excitability could then be ascribed to a change in central nervous system excitability.

\subsubsection{Analysis of H-slopes Stability in Trials 1-2}

The ICC of H-slopes in Trials 1 and 2 of the 8 subjects was 0.9728 . This indicated a high degree of consistency and stability of the $\alpha$-motoneuron pool prior to acupuncture. The means of the H-slopes in Trials 1 and 2 were also compared by using PairedSample T-Test. There was no significant difference between H-slopes of the two control trials $(p=0.293)$. The test suggested insignificant difference in $\alpha$-motoneuron excitability between Trials 1 and 2 . 


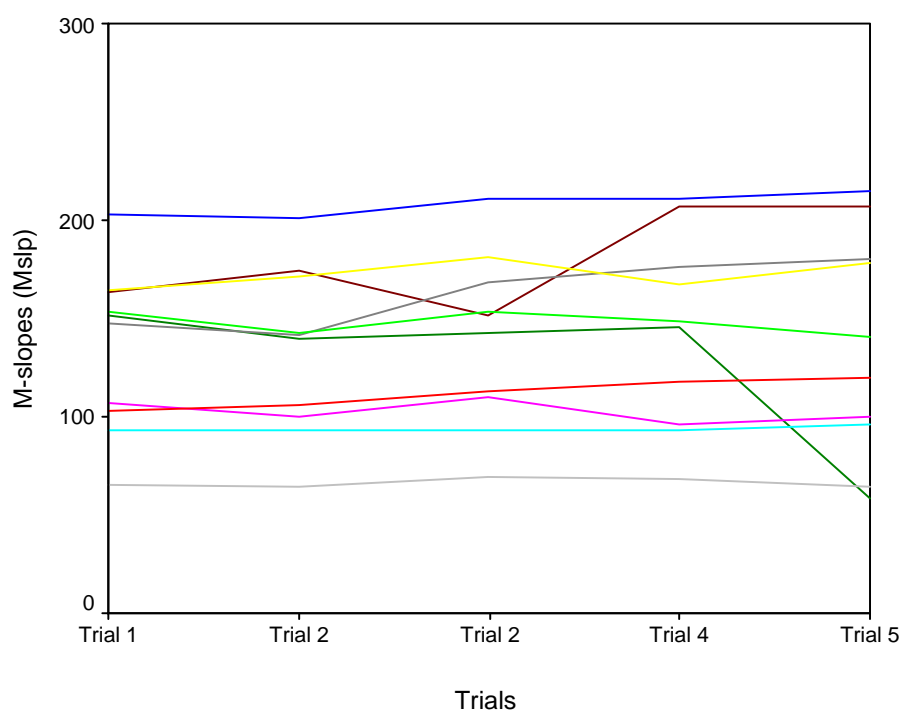

Fig.3.6A Changes in M-slopes Trials 1-5 in short duration acupuncture (extended observation; $n=10$ ). Individual subject's M-slopes from the five trials are shown above. Marked fluctuation in M-slopes during the experiment in two subjects is demonstrated.

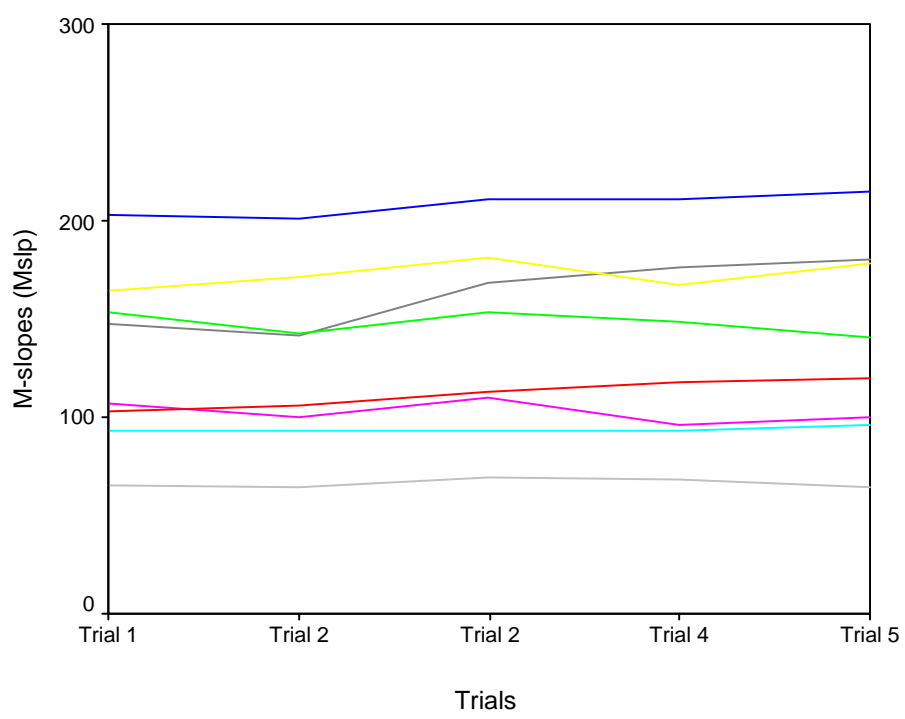

Fig.3.6B Changes in M-slopes from Trials 1-5 in short duration acupuncture (extended observation; $\mathrm{n}=8$ ). Two subjects with non-overlapping $y$-intercepts $95 \%$ confidence intervals were excluded. One subject with non-overlapping $y$-intercepts $95 \%$ confidence intervals was included under special conditions. Only small fluctuations in M-slopes from Trials 1-5 are now apparent. 


\subsubsection{Changes in Motoneuron Excitability}

\subsubsection{Changes in $\mathrm{H}_{\mathrm{slp}}$ after short duration acupuncture (extended observation)}

The mean of the $\mathrm{H}_{\text {slp }}$ in Trials 1 and 2 was calculated and referred to as baseline.

Individual changes in $\mathrm{H}_{\text {slp }}$ during the experiment are shown in Fig. 3.7. The means and standard deviations of $\mathrm{H}_{\text {slp }}$ taken during the experiment were calculated and shown in Table 3.5. The large standard deviations reflected the marked inter-individual differences in baseline $\mathrm{H}_{\text {slp }}$ and individual subject's responses to acupuncture.

To determine significant differences in $\mathrm{H}_{\text {slp }}$ between baseline and Trials $3-5, \mathrm{H}_{\text {slp }}$ from individual subjects were pooled and analysed by repeated-measures ANOVA. There was no significant difference in $\mathrm{H}_{\text {slp }}$ between baseline and Trials 3-5 ( $\left.p=0.834\right)$. Between-subjects effect, however, was found to be significant $(p=0.002)$.

The results indicated that short duration acupuncture had no significant effects on $\alpha$ motoneuron excitability as assessed by comparison of $\mathrm{H}_{\text {slp }}$ up to 30 minutes after the intervention. On the other hand, a wide variation in individual responses to acupuncture was demonstrated. 


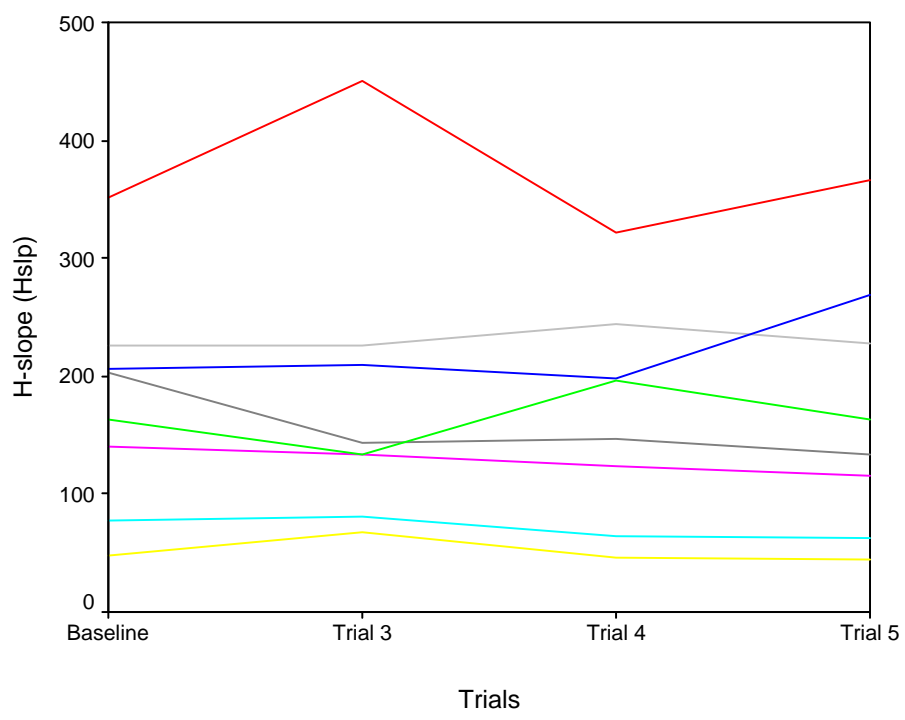

Fig. 3.7 Changes in $\mathrm{H}_{\text {slp }}$ (baseline, Trials 3-5) in short duration acupuncture (extended observation; $\mathrm{n}=8$ ).

Table 3.5 Descriptive statistics of $\mathrm{H}_{\text {slp }}$ (baseline, Trials 3-5) in short duration acupuncture (extended observation; $\mathrm{n}=8$ ).

Descriptive Statistics

\begin{tabular}{|l|r|r|r|r|r|}
\hline & $\mathrm{N}$ & Minimum & Maximum & \multicolumn{1}{c|}{ Mean } & Std. Deviation \\
\hline Baseline $\mathrm{H}_{\text {slp }}$ & 8 & 47.60 & 351.15 & 176.7559 & 94.41977 \\
$\mathrm{H}_{\text {slp Trial 3 }}$ & 8 & 66.97 & 449.97 & 180.7605 & 121.99050 \\
$\mathrm{H}_{\text {slp }}$ Trial 4 & 8 & 46.30 & 321.70 & 167.8368 & 91.83669 \\
$\mathrm{H}_{\text {slp }}$ Trial 5 & 8 & 44.37 & 366.90 & 173.0050 & 109.22695 \\
\hline
\end{tabular}




\subsubsection{Percentage Change in $\mathrm{H}_{\text {slp }}$ after short duration acupuncture (extended}

$\underline{\text { observation) }}$

Percentage changes in Hslp in Trials 3-5 after short duration acupuncture are shown in Fig. 3.8. Variations in individual responses to short duration acupuncture were apparent. The percentage change of $\mathrm{H}_{\text {slp }}$ in Trials 3-5 were pooled for statistical analysis (Table 3.6).

The percentage changes of $\mathrm{H}_{\text {slp }}$ in Trials 3-5 were subjected to One-Sample T-Test analysis. The percentage changes of $\mathrm{H}_{\text {slp }}$ in Trial $3(p=0.719)$, Trial $4(p=0.355)$ and Trial $5(p=0.478)$ were not significant. This indicated that there was no significant difference in $\alpha$-motoneuron excitability from baseline measured by percentage change in $\mathrm{H}_{\mathrm{slp}}$ as a result of short duration acupuncture up to 30 minutes after the intervention. Percentage changes of $\mathrm{H}_{\text {slp }}$ in trial 3,4 and 5 were then compared using repeated measures ANOVA. There was no significant difference in percentage change in $\mathrm{H}_{\text {slp }}$ among the three trials $(p=0.520)$, which indicated the absence of significant change in motoneuron excitability between Trials 3-5. 


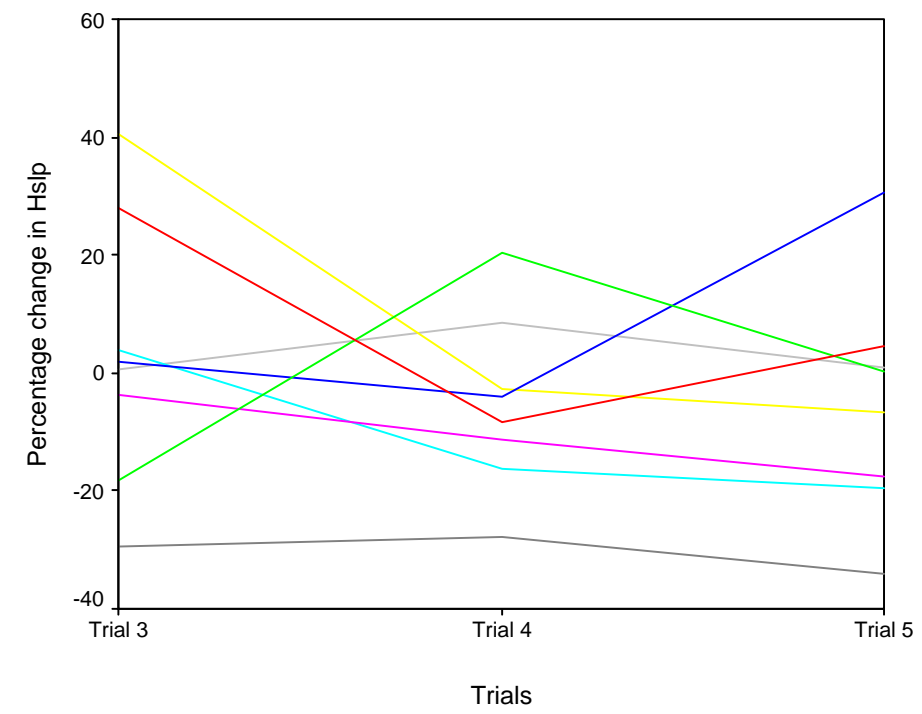

Fig. 3.8 Percentage changes in $\mathrm{H}_{\text {sip }}$ in Trials 3-5 after short duration acupuncture (extended observation; $\mathrm{n}=8$ ).

Table 3.6 Descriptive statistics of percentage changes in $\mathrm{H}_{\mathrm{slp}}$ in Trials 3-5 after short duration acupuncture (extended observation; $n=8$ ).

\section{Descriptive Statistics}

\begin{tabular}{|l|r|r|r|r|r|}
\hline & $\mathrm{N}$ & Minimum & Maximum & \multicolumn{1}{c|}{ Mean } & Std. Deviation \\
\hline \%change $\mathrm{H}_{\text {slp }}$ trial 3 & 8 & -29.34 & 40.67 & 3.0067 & 22.68810 \\
\%change $\mathrm{H}_{\text {slp }}$ trial 4 & 8 & -27.82 & 20.47 & -5.1938 & 14.83757 \\
\%change $\mathrm{H}_{\text {slp }}$ trial 5 & 8 & -33.94 & 30.73 & -5.1427 & 19.40731 \\
\hline
\end{tabular}




\subsubsection{Change in $\mathrm{H}_{\mathrm{slp}} \underline{\mathrm{M}} \underline{\mathrm{M}} \underline{\mathrm{slp}}$ after short duration acupuncture (extended observation)}

The mean of $\mathrm{H}_{\mathrm{slp}} / \mathrm{M}_{\text {slp }}$ in Trials 1 and 2 was calculated and referred to as baseline. Individual changes in $\mathrm{H}_{\text {slp }} / \mathrm{M}_{\text {slp }}$ during the experiment are shown in Fig. 3.9. The means and standard deviations of $\mathrm{H}_{\mathrm{slp}} / \mathrm{M}_{\text {slp }}$ during the experiment were calculated and shown in Table 3.7. Inter-individual differences in baseline $\mathrm{H}_{\mathrm{slp}} / \mathrm{M}_{\mathrm{slp}}$ and responses to acupuncture were evident from the large standard deviations in comparison to the means.

To determine significant differences in $\mathrm{H}_{\text {slp }} / \mathrm{M}_{\text {slp }}$ between baseline and Trials 3-5, $\mathrm{H}_{\text {slp }} / \mathrm{M}_{\text {slp }}$ from individual subjects were pooled and analysed by repeated measures ANOVA. There was no significant difference in $\mathrm{H}_{\mathrm{slp}} / \mathrm{M}_{\text {slp }}$ between the baseline and Trials 3-5 $(p=0.608)$. Between-subjects effect, however, was found to be significant ( $p$ $=0.008)$. The results indicated that short duration acupuncture had no significant effects on $\alpha$-motoneuron excitability up to 30 minutes after the intervention as assessed by comparison of $\mathrm{H}_{\mathrm{slp}} / \mathrm{M}_{\mathrm{slp}}$. On the other hand, a wide variation in individual responses to acupuncture was demonstrated. 


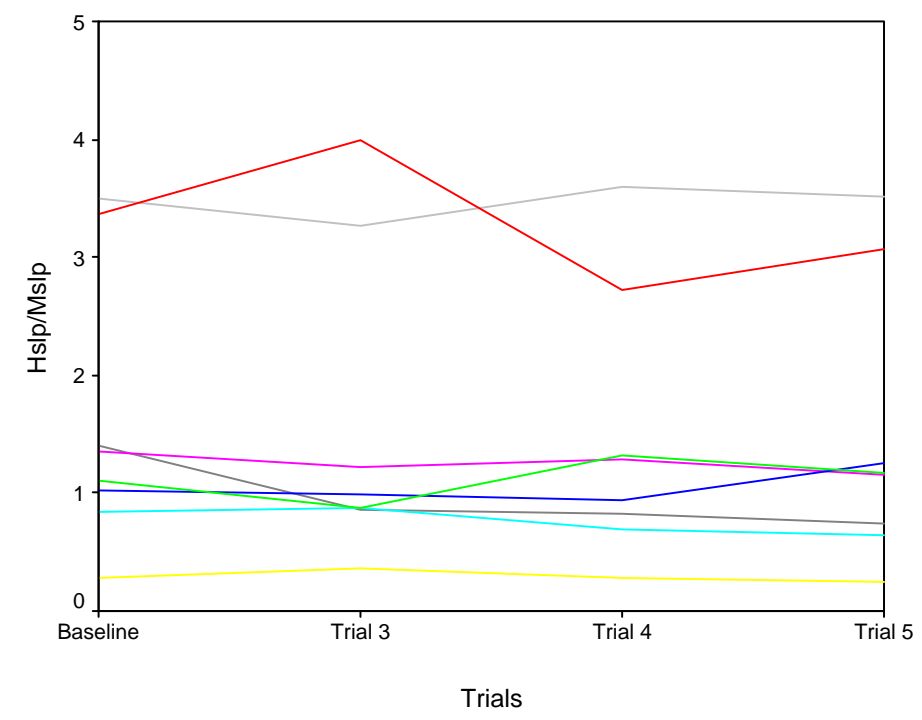

Fig. 3.9 Changes in $\mathrm{H}_{\text {slp }} / \mathrm{M}_{\text {slp }}$ (baseline, Trials 3-5) in short duration acupuncture (extended observation; $\mathrm{n}=8$ ).

Table 3.7 Descriptive statistics of $\mathrm{H}_{\text {slp }} / \mathrm{M}_{\text {slp }}$ (baseline, Trials 3-5) in short acupuncture (extended observation; $\mathrm{n}=8$ ).

\section{Descriptive Statistics}

\begin{tabular}{|l|r|r|r|r|r|}
\hline & $\mathrm{N}$ & Minimum & Maximum & Mean & Std. Deviation \\
\hline $\mathrm{H}_{\text {slp }} / \mathrm{M}_{\text {slp }}$ Baseline & 8 & .28 & 3.49 & 1.6077 & 1.17795 \\
$\mathrm{H}_{\text {slp }} / \mathrm{M}_{\text {slp }}$ Trial 3 & 8 & .37 & 3.99 & 1.5552 & 1.31634 \\
$\mathrm{H}_{\text {slp }} / \mathrm{M}_{\text {slp }}$ Trial 4 & 8 & .28 & 3.60 & 1.4602 & 1.12613 \\
$\mathrm{H}_{\text {slp }} / \mathrm{M}_{\text {sIp }}$ Trial 5 & 8 & .25 & 3.51 & 1.4743 & 1.17415 \\
\hline
\end{tabular}




\subsubsection{Percentage Change in $\mathrm{H}_{\underline{s l p}} \underline{\mathrm{M}_{\text {slp }}} \underline{\text { after short duration acupuncture (extended }}$ $\underline{\text { observation) }}$}

Percentage changes in $\mathrm{H}_{\mathrm{slp}} / \mathrm{M}_{\text {slp }}$ in Trials 3-5 after short duration acupuncture are shown in Fig. 3.10. Variations in individual responses to short duration acupuncture were apparent. The percentage change of $\mathrm{H}_{\mathrm{slp}} / \mathrm{M}_{\mathrm{slp}}$ in Trials 3-5 were pooled for statistical analysis (Table 3.8).

The percentage changes of $\mathrm{H}_{\mathrm{slp}} / \mathrm{M}_{\text {slp }}$ in Trials 3-5 were subjected to One-Sample T-Test analysis. The percentage changes of $\mathrm{H}_{\mathrm{slp}} / \mathrm{M}_{\mathrm{slp}}$ in Trial $3(p=0.693)$, Trial $4(p=0.215)$ or Trial $5(p=0.233)$ were not significant. This indicated that there was no significant difference in $\alpha$-motoneuron excitability from baseline measured by percentage change in $\mathrm{H}_{\mathrm{slp}} / \mathrm{M}_{\mathrm{slp}}$ as a result of short duration acupuncture up to 30 minutes after the intervention. The percentage change of $\mathrm{H}_{\mathrm{slp}} / \mathrm{M}_{\text {slp }}$ in Trials 3-5 were also compared using repeated-measures ANOVA. There was no significant difference in percentage change of $\mathrm{H}_{\mathrm{slp}} / \mathrm{M}_{\text {slp }}$ between the three trials $(p=0.695)$, which indicated the absence of significant change in motoneuron excitability between Trials 3-5.

\subsubsection{Conclusion}

There was no significant change in $\alpha$-motoneuron excitability up to 30 minutes after short duration acupuncture as assessed by changes in $\mathrm{H}_{\text {slp }}$ and $\mathrm{H}_{\mathrm{slp}} / \mathrm{M}_{\text {slp }}$, or percentage changes in $\mathrm{H}_{\text {slp }}$ and $\mathrm{H}_{\text {slp }} / \mathrm{M}_{\text {slp. }}$ A wide variation in individual responses to short duration acupuncture was observed. 


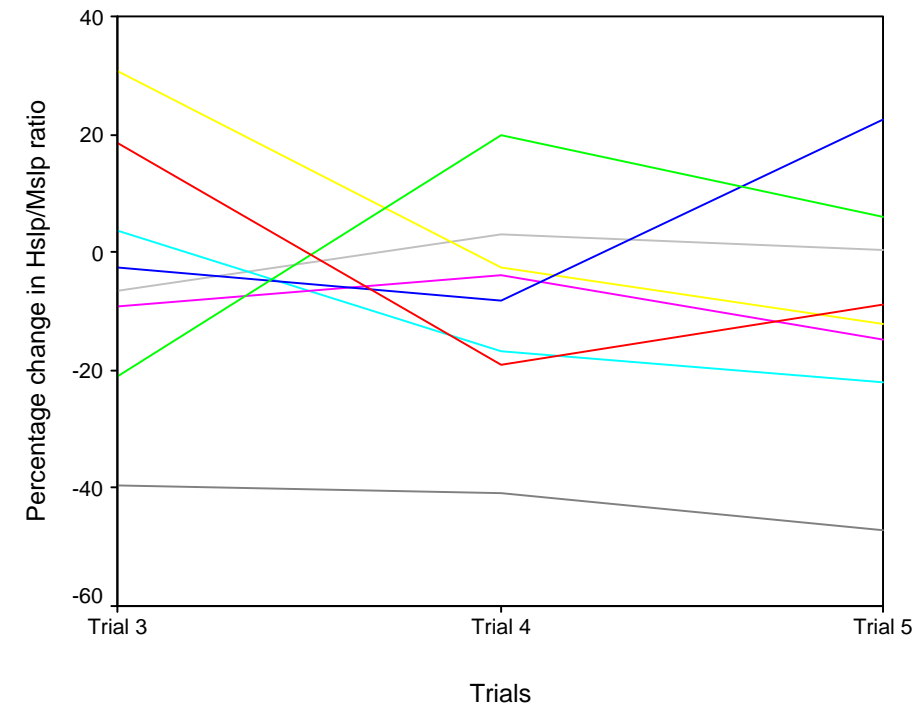

Fig. 3.10 Percentage changes in $H_{\text {slp }} / M_{\text {slp }}$ in Trials 3-5 after short duration acupuncture (extended observation; $\mathrm{n}=8$ ).

Table 3.8 Descriptive statistics of percentage changes in $\mathrm{H}_{\text {slp }} / \mathrm{M}_{\text {slp }}$ in Trials 3-5 after short duration acupuncture (extended observation; $n=8$ ).

One-Sample Statistics

\begin{tabular}{|l|r|r|r|r|}
\hline & $\mathrm{N}$ & Mean & Std. Deviation & $\begin{array}{c}\text { Std. Error } \\
\text { Mean }\end{array}$ \\
\hline \% change $\mathrm{H}_{\text {slp }} / \mathrm{M}_{\text {slp }}$ Trial 3 & 8 & -3.1871 & 21.88776 & 7.73849 \\
\% change $\mathrm{H}_{\text {slp }} / \mathrm{M}_{\text {slp }}$ Trial 4 & 8 & -8.5918 & 17.81083 & 6.29708 \\
\% change $\mathrm{H}_{\text {slp }} / \mathrm{M}_{\text {slp }}$ Trial 5 & 8 & -9.5107 & 20.60442 & 7.28476 \\
\hline
\end{tabular}




\subsection{Effects of Sustained Acupuncture}

To determine the effects of sustained acupuncture on $\alpha$-motoneuron excitability, the subjects received manual acupuncture for 20 minutes. Timing began when the subject reported the appearance of acupuncture sensation (deqi). H-reflexes were collected before, immediately following and then 15 minutes after withdrawal of the acupuncture needles.

\subsubsection{M-slope stability}

\subsubsection{Analysis of M-slope Stability in Trials 1-4 (Preliminary)}

M-slope data in Trials 1 to 4 from all subjects are shown in Fig. 3.11A. In some subjects, a marked fluctuation in the M-slope across the four trials indicated unstable peripheral conditions. The ICC of the M-slopes across Trials 1 to 4 was 0.9135 . This was below the criteria $(\mathrm{ICC}=0.95)$ set for stability $($ Refer section 2.6.3). Therefore, the M-slopes from all trials were subjected to further analysis.

\subsubsection{Analysis of M-slope Stability in Trials 1-4 (Intercept Method)}

Four (4) subjects had non-overlapping 95\% confidence intervals when examined by the Intercept Method (Kerr et al., 2002b). Data from these four subjects were excluded. The results of the intercept confidence intervals analysis are presented in Appendix VI.

Intraclass correlation analysis of M-slopes from Trials 1 to 4 was repeated for the remaining 16 subjects. The ICC was found to have improved from the original 0.9135 to 0.9662. Repeated measures ANOVA of M-slopes from Trials 1 to 4 also showed no significant difference between them $(p=0.813)$. There is now much less within-subject M-slope variation among the remaining subjects (Fig. $3.11 \mathrm{~B}$ ). Results of the tests 
reflected highly consistent M-slopes from Trials 1 to 4 and indirectly confirmed stable peripheral conditions during the experiments in these 16 subjects. Changes in the $\alpha-$ motoneuron excitability could then be ascribed to a change in central nervous system excitability. 


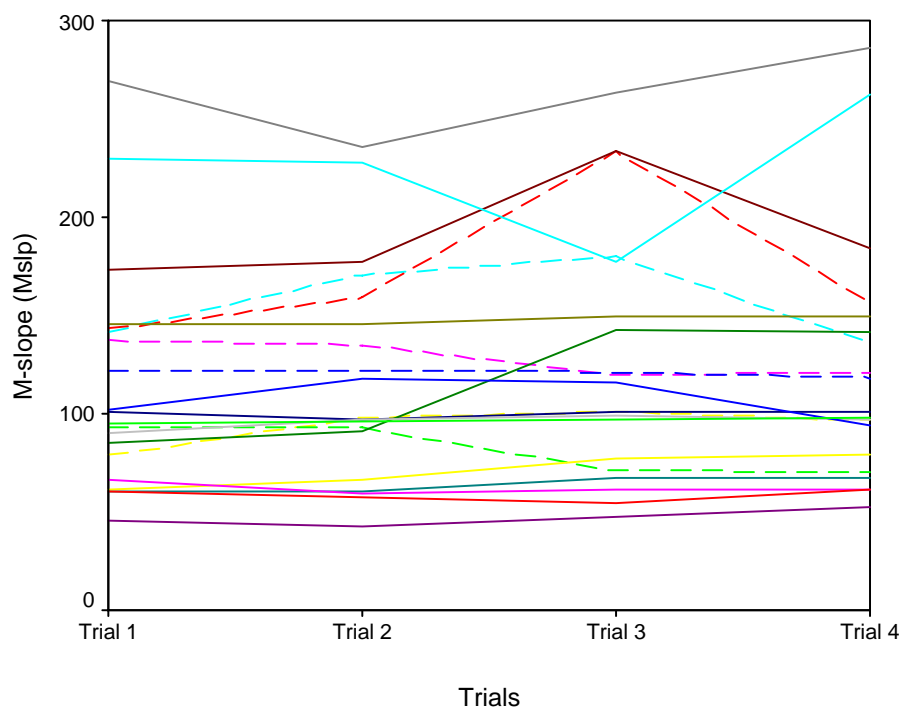

Fig. 3.11A Changes in $M_{\text {sip }}$ in Trials 1-4 in sustained acupuncture $(n=20)$. Individual subject's M-slopes from the four trials are shown. Marked fluctuation in M-slopes during the experiment in some subjects is demonstrated.

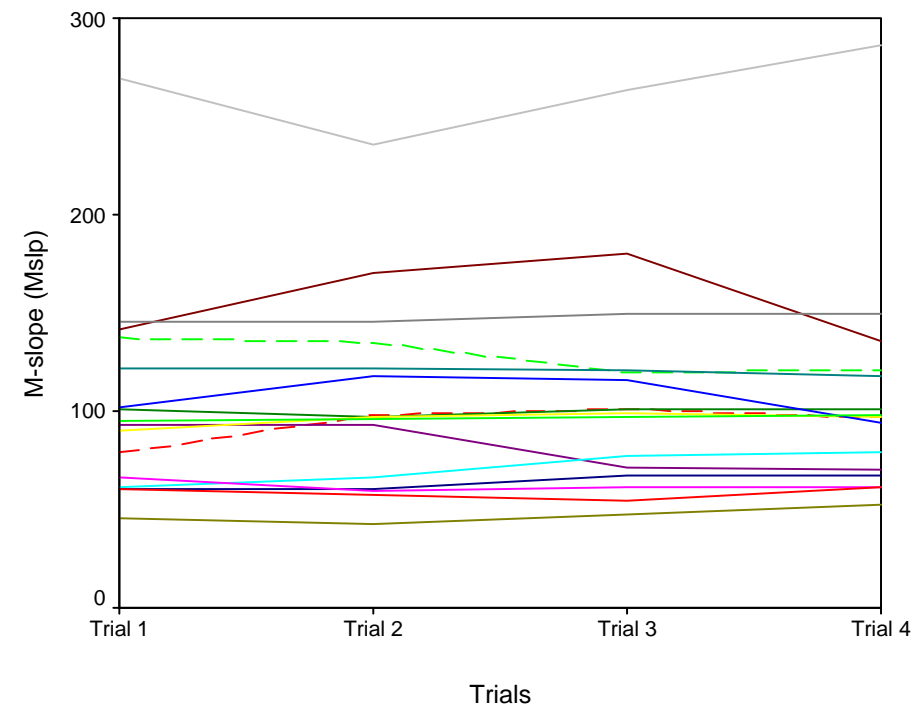

Fig. 3.11B Changes in $M_{\text {slp }}$ in Trials 1-4 in sustained acupuncture $(n=16)$. Data from 4 subjects with non-overlapping $y$-intercepts $95 \%$ confidence intervals were excluded. Only small fluctuations in M-slopes from Trials 1-4 are now apparent. 


\subsubsection{Analysis of $\mathbf{H}$-slopes Stability in Trials 1-2}

The means of the H-slopes in Trials 1 and 2 were compared by using Paired-Sample TTest. There was no significant difference between the H-slopes of Trials 1 and $2(p=$ 0.458). Although this suggested insignificant difference in $\alpha$-motoneuron excitability between Trials 1 and 2, one subject had marked change in H-slope (Fig.3.12A). The ICC of the H-slopes in Trials 1 and 2 of the 16 subjects was 0.8621 . This was below the criteria $(\mathrm{ICC}=0.95)$ set for stability (Refer section 2.6.3). In order to ensure preintervention H-slopes stability and repeatability, the set of data with marked change in $\mathrm{H}$-slope was discarded. The ICC of H-slopes in Trials 1 and 2 was improved from 0.8621 to 0.9609 , which reflected stable baseline in the remaining 15 participants (Fig. 3.12B). Data from these 15 subjects were then used for final analysis of the effects of sustained acupuncture on $\alpha$-motoneuron excitability. The ICC of M-waves of these 15 subjects was 0.9635 , which was above the criteria set for stability in this study. 


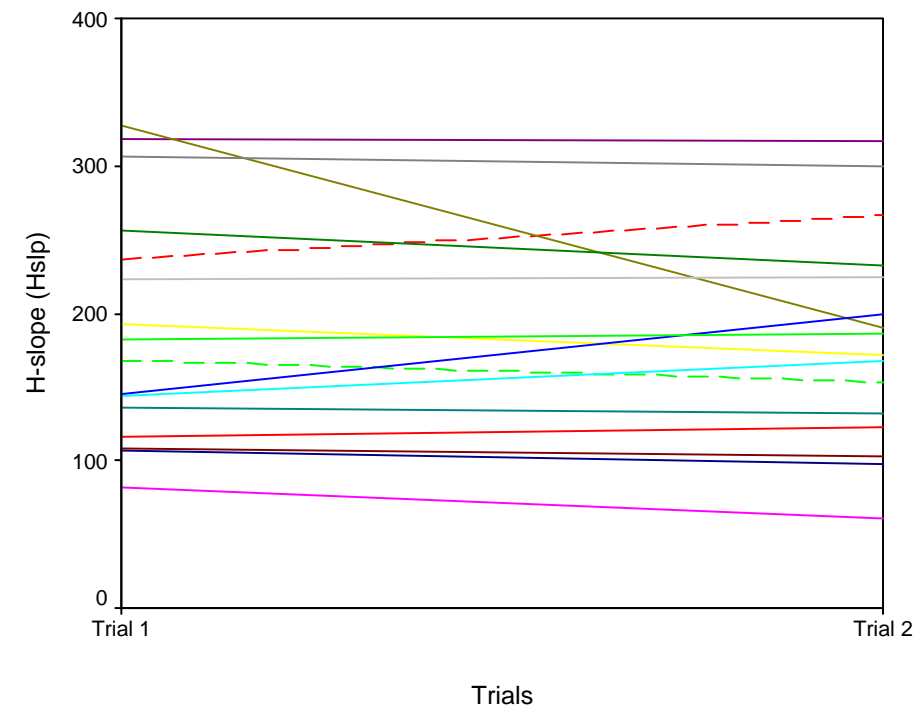

Fig. 3.12A Changes in $\mathrm{H}_{\text {sip }}$ in Trials $1-2$ in sustained acupuncture $(\mathrm{n}=16)$. Individual subject's $\mathrm{H}_{\text {slp }}$ from Trials 1-2 are shown. One subject showed marked reduction in $\mathrm{H}_{\text {slp }}$ in Trial 2.

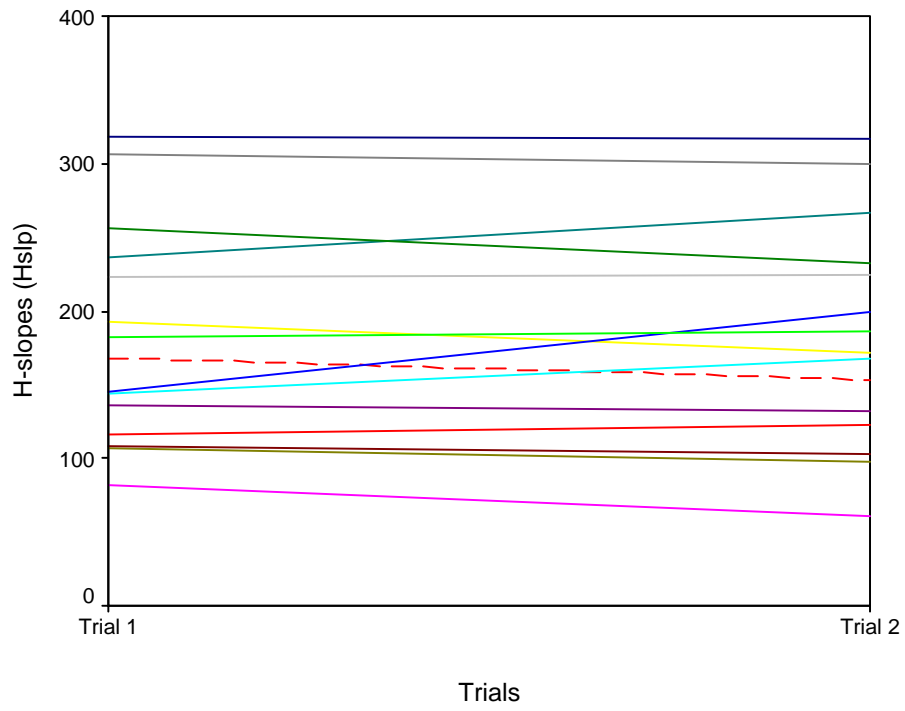

Fig. 3.12B Changes in $\mathrm{H}_{\mathrm{slp}}$ in Trials 1-2 in sustained acupuncture $(\mathrm{n}=15)$. Data from the subject who showed marked reduction of $\mathrm{H}_{\text {slp }}$ in Trial 2 was discarded. The $\mathrm{H}$-slopes are now more consistent and stable in the remaining 15 subjects. 


\subsubsection{Changes in Motoneuron Excitability}

\subsubsection{Changes in $\mathrm{H}_{\text {slp }}$ after sustained acupuncture}

The mean of the $\mathrm{H}_{\text {slp }}$ in Trials 1 and 2 was calculated and referred to as baseline. Individual changes in $\mathrm{H}_{\text {slp }}$ during the experiment are shown in Fig. 3.13. The means and standard deviations of $\mathrm{H}_{\text {slp }}$ taken during the experiment were calculated and shown in Table 3.9. The large standard deviations reflected the marked inter-individual differences in baseline $\mathrm{H}_{\text {slp }}$ and individual subject's responses to acupuncture.

To determine significant differences in $\mathrm{H}_{\text {slp }}$ between baseline and Trials $3-4, \mathrm{H}_{\text {slp }}$ from individual subjects were pooled and analysed by repeated-measures ANOVA. The test showed a significant difference in $\mathrm{H}_{\text {slp }}$ between baseline and Trials 3-4 $(p=0.038)$. Between-subjects effect was also found to be significant $(p<0.0005)$. The results indicated that sustained acupuncture had significant effects on $\alpha$-motoneuron excitability as assessed by comparison of $\mathrm{H}_{\text {slp }}$. A wide variation in individual responses to acupuncture was also demonstrated. 


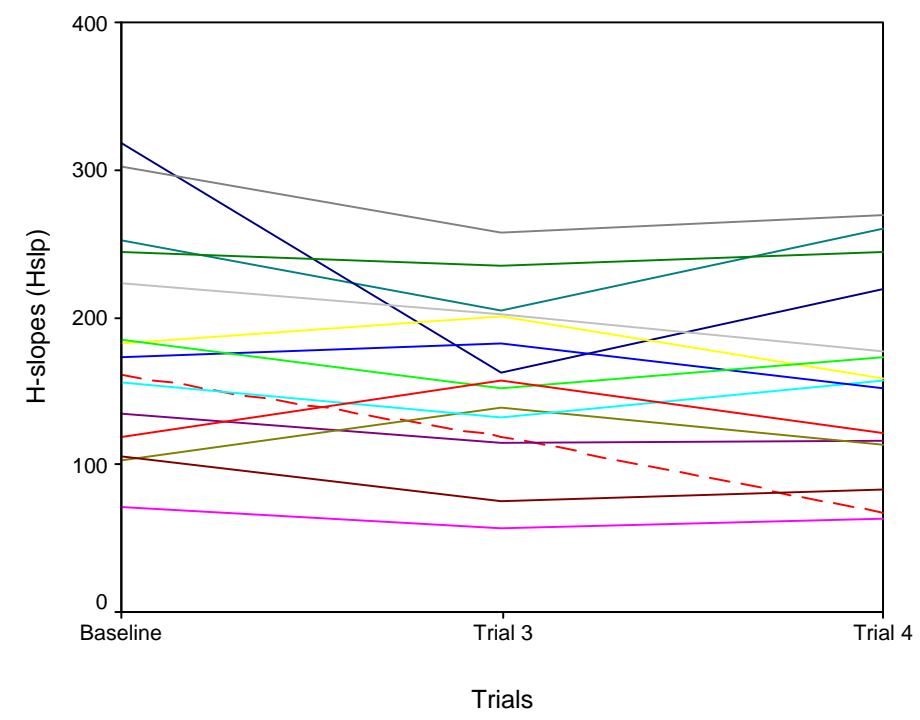

Fig. 3.13 Changes in $\mathrm{H}_{\text {slp }}$ (baseline, Trials 3-4) in sustained acupuncture $(n=15)$. Marked inter-individual variability in baseline $H_{\text {slp }}$ and response to acupuncture at trial 3 and 4 in different subjects is demonstrated.

Table 3.9 Descriptive statistics of $\mathrm{H}_{\text {slp }}$ (baseline, trials 3 and 4) in sustained acupuncture $(n=15)$.

Descriptive Statistics

\begin{tabular}{|l|r|r|r|r|r|}
\hline & \multicolumn{1}{|c|}{$\mathrm{N}$} & Minimum & Maximum & \multicolumn{1}{c|}{ Mean } & Std. Deviation \\
\hline $\mathrm{H}_{\text {slp }}$ Baseline & 15 & 71.40 & 317.56 & 181.9108 & 73.27736 \\
$\mathrm{H}_{\text {slp }}$ Trial 3 & 15 & 56.57 & 257.66 & 159.2913 & 55.97252 \\
$\mathrm{H}_{\text {slp }}$ Trial 4 & 15 & 63.49 & 269.43 & 158.3559 & 66.72641 \\
\hline
\end{tabular}




\subsubsection{Percentage Change in $\mathrm{H}_{\text {slp }}$ after sustained acupuncture}

Percentage changes in $\mathrm{H}_{\text {slp }}$ in Trials 3 and 4 after sustained acupuncture are shown in Fig. 3.14. Variations in individual responses to sustained acupuncture were apparent. The percentage change in $\mathrm{H}_{\text {slp }}$ in Trials 3 and 4 were pooled for statistical analysis (Table 3.10). There appeared to be a negative percentage change in $\mathrm{H}_{\text {slp }}$ immediately after sustained acupuncture (-9\%) followed by a further reduction 15 minutes later $(-12 \%)$. The percentage change in $\mathrm{H}_{\text {slp }}$ in Trials 3 and 4 were subjected to One-Sample T-Test analysis. The percentage change of $\mathrm{H}_{\text {slp }}$ in Trial $3(p=0.134)$ was not significant but that in Trial $4(p=0.015)$ was significant. This indicated that there was no significant difference in $\alpha$-motoneuron excitability from baseline immediately after sustained acupuncture, but a significant difference was recorded 15 minutes later. The percentage changes in $\mathrm{H}_{\text {slp }}$ in Trials 3 and 4 were also compared using Paired-Sample Ttest. There was no significant difference between the two trials $(p=0.31)$, which indicated the absence of significant change in motoneuron excitability between Trials 34. This could be accounted for by the wide standard deviation in each trial. 


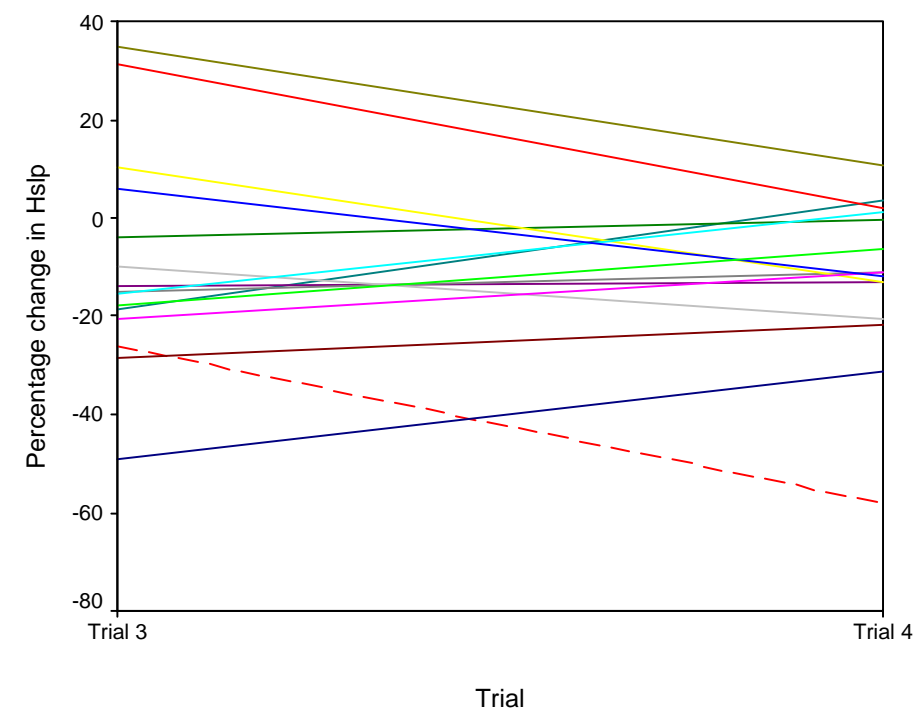

Fig. 3.14

Percentage changes in $\mathrm{H}_{\text {slp }}$ in Trials 3-4 after sustained acupuncture $(n=15)$.

Table 3.10 Descriptive statistics of percentage changes in $\mathrm{H}_{\text {slp }}$ in Trials 3-4 after sustained acupuncture $(n=15)$.

\section{Descriptive Statistics}

\begin{tabular}{|l|r|r|r|r|r|}
\hline & \multicolumn{1}{|c|}{$\mathrm{N}$} & Minimum & Maximum & \multicolumn{1}{c|}{ Mean } & Std. Deviation \\
\hline \%change $\mathrm{H}_{\text {slp }}$ Trial 3 & 15 & -48.93 & 34.83 & -9.0907 & 22.12071 \\
\%change $\mathrm{H}_{\text {slp }}$ Trial 4 & 15 & -58.12 & 10.81 & -12.0799 & 16.83675 \\
\hline
\end{tabular}




\subsubsection{Change in $\mathrm{H}_{\mathrm{slp}} \underline{\mathrm{M}}_{\mathrm{slp}}$ after sustained acupuncture}

The mean of $\mathrm{H}_{\mathrm{slp}} / \mathrm{M}_{\mathrm{slp}}$ in Trials 1 and 2 was calculated and referred to as baseline. Individual changes in Hslp/Mslp during the experiment are shown in Fig. 3.15. The means and standard deviations of $\mathrm{H}_{\mathrm{slp}} / \mathrm{M}_{\text {slp }}$ during the experiment were calculated and shown in Table 3.11. Inter-individual differences in baseline $\mathrm{H}_{\mathrm{slp}} / \mathrm{M}_{\mathrm{slp}}$ and responses to acupuncture were evident from the large standard deviation in comparison to the mean.

To determine significant differences in $\mathrm{H}_{\text {slp }} / \mathrm{M}_{\text {slp }}$ between baseline and Trials 3 and 4 , $\mathrm{H}_{\text {slp }} / \mathrm{M}_{\text {slp }}$ from individual subjects were pooled and analysed by repeated-measures ANOVA. There was significant difference in $\mathrm{H}_{\text {slp }} / \mathrm{M}_{\text {slp }}$ between baseline and Trials 3 and $4(p=0.006)$. Between-subjects effect was also found to be significant $(p<$ 0.0005). The results indicated that sustained acupuncture had significant effects on $\alpha$ motoneuron excitability as assessed by comparison of $\mathrm{H}_{\text {slp }} / \mathrm{M}_{\text {slp }}$. A wide variation in individual responses to acupuncture was also demonstrated. 


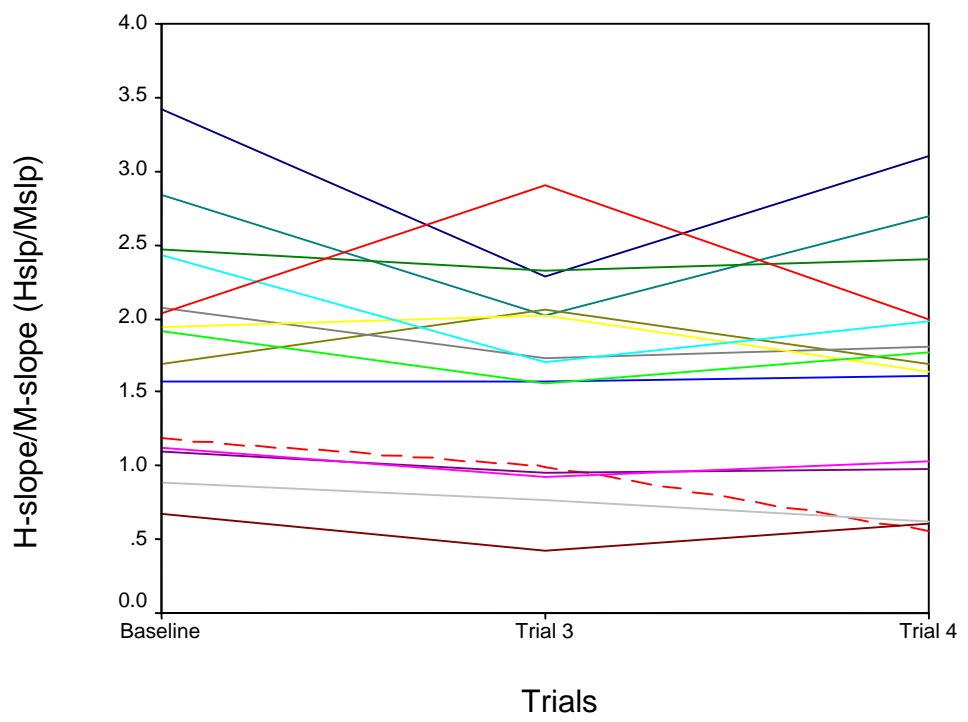

Fig. 3.15 Changes in $\mathrm{H}_{\text {slp }} / \mathrm{M}_{\text {slp }}$ (baseline, Trials 3-4) in sustained acupuncture $(n=15)$.

Table 3.11 Descriptive statistics of $\mathrm{H}_{\text {slp }} / \mathrm{M}_{\text {slp }}$ (baseline, Trials 3-4) in sustained acupuncture $(n=15)$.

Descriptive Statistics

\begin{tabular}{|l|r|r|r|r|r|}
\hline & $\mathrm{N}$ & Minimum & Maximum & Mean & Std. Deviation \\
\hline $\mathrm{H}_{\text {slp }} / \mathrm{M}_{\text {slp }}$ Baseline & 15 & .68 & 3.42 & 1.8242 & .76447 \\
$\mathrm{H}_{\text {slp }} / \mathrm{M}_{\text {slp }}$ Trial 3 & 15 & .42 & 2.90 & 1.6146 & .68584 \\
$\mathrm{H}_{\text {slp }} / \mathrm{M}_{\text {slp }}$ Trial 4 & 15 & .56 & 3.10 & 1.6317 & .76574 \\
\hline
\end{tabular}




\subsubsection{Percentage Change in $\mathrm{H}_{\mathrm{slp}} \underline{\mathrm{M}} \underline{\mathrm{s}} \underline{\text { after sustained acupuncture }}$}

Percentage changes in $\mathrm{H}_{\text {slp }} / \mathrm{M}_{\text {slp }}$ in Trials 3 and 4 after sustained acupuncture are shown in Fig. 3.16. Variations in individual responses to sustained acupuncture were apparent. The percentage change of $\mathrm{H}_{\text {slp }} / \mathrm{M}_{\text {slp }}$ in Trials 3 and 4 were pooled for statistical analysis (Table 3.12). There appeared to be a negative percentage change in $H_{s l p} / M_{\text {slp }}$ immediately after sustained acupuncture $(-10.9 \%)$ followed by a further reduction 15 minutes later $(-12.3 \%)$. The percentage changes of $\mathrm{H}_{\mathrm{slp}} / \mathrm{M}_{\text {slp }}$ in Trials 3 and 4 were subjected to One-Sample T-Test analysis. The percentage change of $\mathrm{H}_{\mathrm{slp}} / \mathrm{M}_{\text {slp }}$ in Trial 3 was not significant $(p=0.069)$, but that in Trial $4(p=0.004)$ was significant. This indicated that, as a result of sustained acupuncture, there was significant difference in $\alpha-$ motoneuron excitability from baseline measured by percentage change in $\mathrm{H}_{\text {slp }} / \mathrm{M}_{\text {slp }} 15$ minutes after sustained acupuncture. The percentage change of $\mathrm{H}_{\mathrm{slp}} / \mathrm{M}_{\mathrm{slp}}$ in Trials 3 and 4 were also compared using Paired-Sample T-test. There was no significant difference between the two trials $(p=0.543)$, which indicated the absence of significant change in motoneuron excitability between Trials 3 and 4 . This could be accounted for by the wide standard deviation in each trial.

\subsubsection{Conclusion}

After sustained acupuncture, there was a significant change in $\alpha$-motoneuron excitability when assessed by changes in $\mathrm{H}_{\text {slp }}$ and $\mathrm{H}_{\text {slp }} / \mathrm{M}_{\text {slp. }}$. Percentage changes in $\mathrm{H}_{\text {slp }}$ and $\mathrm{H}_{\mathrm{slp}} / \mathrm{M}_{\text {slp }}$ were significant at 15 minutes after completion of acupuncture. A wide variation in individual responses to sustained acupuncture was observed. 


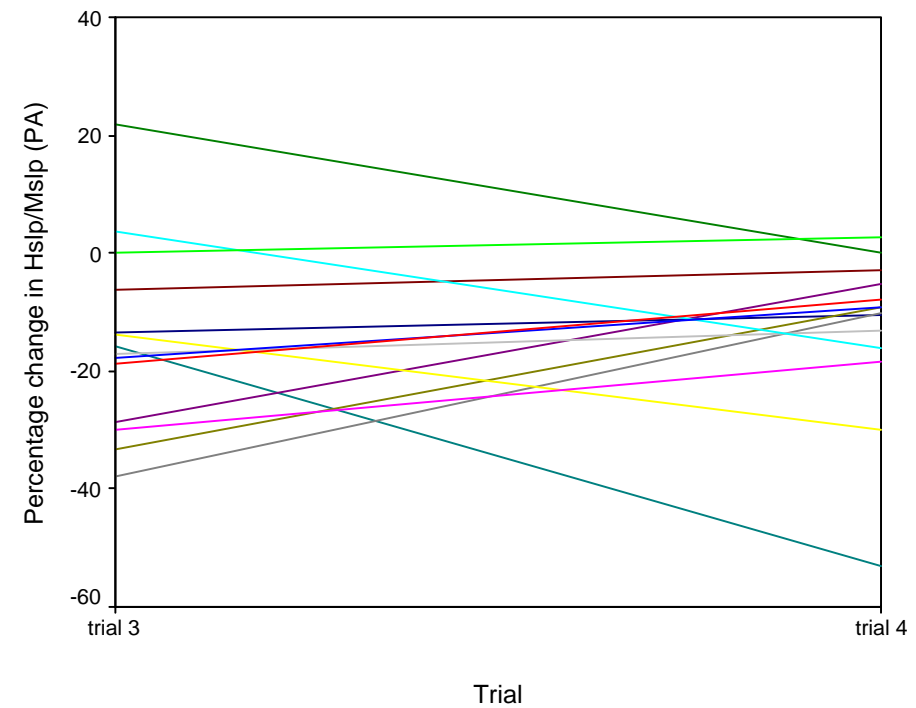

Fig. 3.16 Percentage changes in $\mathrm{H}_{\text {slp }} / \mathrm{M}_{\text {slp }}$ in Trials 3-4 after sustained acupuncture $(n=15)$.

Table 3.12 Descriptive statistics of percentage changes in $H_{s \mid p} / M_{s \mid p}$ in Trials 3-4 after sustained acupuncture $(n=15)$.

\section{Descriptive Statistics}

\begin{tabular}{|l|r|r|r|r|r|}
\hline & N & Minimum & Maximum & \multicolumn{1}{|c|}{ Mean } & Std. Deviation \\
\hline \%change $\mathrm{H}_{\text {slp }} / \mathrm{M}_{\text {slp }}$ Trial 3 & 15 & -37.98 & 42.92 & -10.9208 & 21.43103 \\
\%change $\mathrm{H}_{\text {slp }} / \mathrm{M}_{\text {slp }}$ Trial 4 & 15 & -52.97 & 2.77 & -12.3066 & 13.89951 \\
\hline
\end{tabular}




\subsection{Effects of Sustained Acupuncture (extended observation)}

To determine delayed and/or continual effects of sustained acupuncture on $\alpha$ motoneuron excitability, 10 participants receiving sustained acupuncture had H-reflexes monitored up to 30 minutes after acupuncture. Timing began when the subject reported the appearance of acupuncture sensation (deqi). H-reflexes were collected before, immediately following and then 15 and 30 minutes after withdrawal of the acupuncture needles.

\subsubsection{M-slope stability}

\subsubsection{Analysis of M-slope Stability in Trials 1-5 (Preliminary)}

M-slope data from all subjects in Trials 1 to 5 are shown in Fig. 3.17A. In two subjects, marked fluctuations in the M-slope within the five trials indicated unstable peripheral conditions. The ICC of the M-slopes across Trials 1-5 was 0.8745 . This was below the criteria $(\mathrm{ICC}=0.95)$ set for stability (Refer section 2.6.3). Therefore, the M-slopes from all trials were subjected to further analysis.

\subsubsection{Analysis of M-slope Stability in Trials 1-5 (Intercept Method)}

One (1) subject had non-overlapping $95 \%$ confidence intervals when examined by the Intercept Method (Kerr et al., 2002b). Data from this subject was, therefore, excluded from further study. The results of the intercept confidence intervals analysis are presented in Appendix VI.

Intraclass correlation analysis of M-slopes from Trials 1-5 was repeated for the remaining 9 subjects. After exclusion of data from one subject with non-overlapping 95\% confidence intervals of the $y$-intercepts, the ICC was found to have improved from the original 0.8745 to 0.9339 . However, there was still one subject with obvious 
unstable M-waves in Fig. 3.17B. Data from this subject was also excluded. Intraclass correlation analysis of M-slopes from Trials 1-5 was repeated for the remaining 8 subjects. The ICC was found to have improved further from 0.9339 to 0.9581 . Repeated measures ANOVA of M-slopes from Trials 1-5 also showed no significant difference between them $(p=0.963)$. There is now much less within-subject M-slope variation among the remaining subjects (Fig. 3.17C). Results of the tests reflected highly consistent M-slopes from Trials 1-5 and indirectly confirmed stable peripheral conditions during the experiments in these 8 subjects. Changes in the $\alpha$-motoneuron excitability could then be ascribed to a change in central nervous system excitability. 


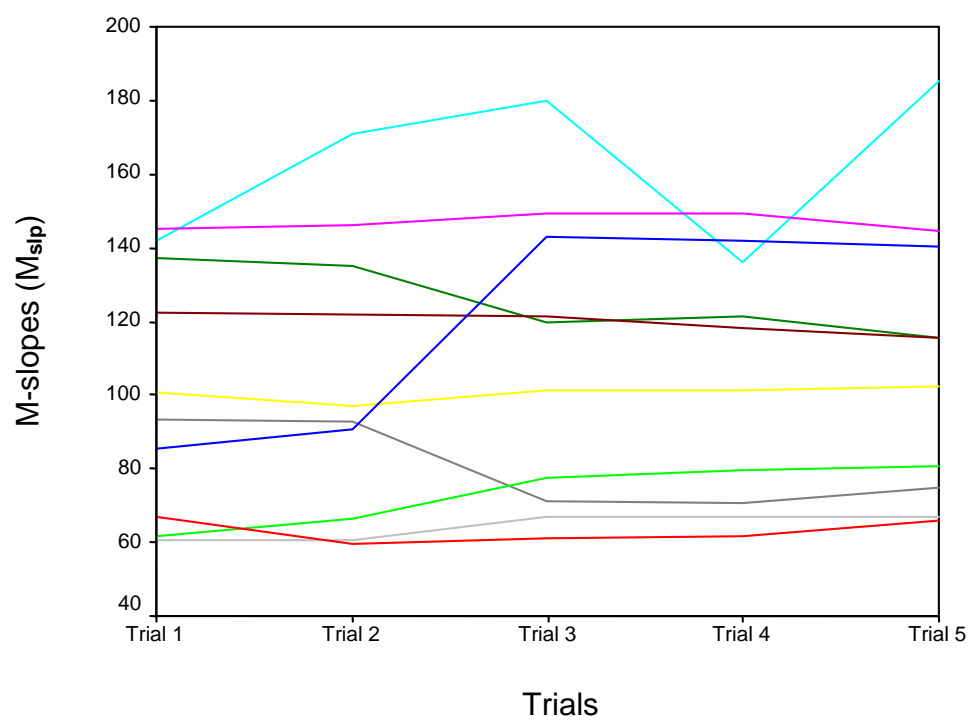

Fig. 3.17A Changes in M-slopes in Trials 1-5 in sustained acupuncture (extended observation; $\mathrm{n}=10$ ). Individual subject's M-slopes from the five trials are shown. Marked fluctuation in M-slopes during the experiment in some subjects is demonstrated.

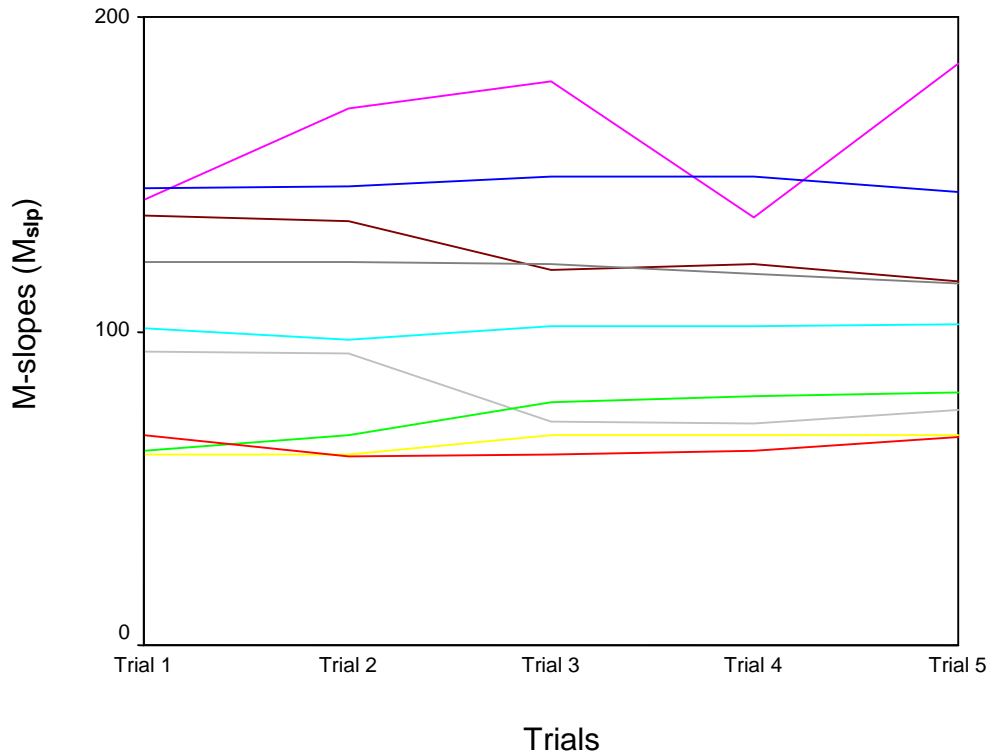

Fig.3.17B Changes in M-slopes in Trials 1-5 in sustained acupuncture (extended observation; $n=9$ ). Data from one subject was already excluded using the $y$-intercept method. However, marked variation in M-slopes in one of the remaining subjects was still apparent. 


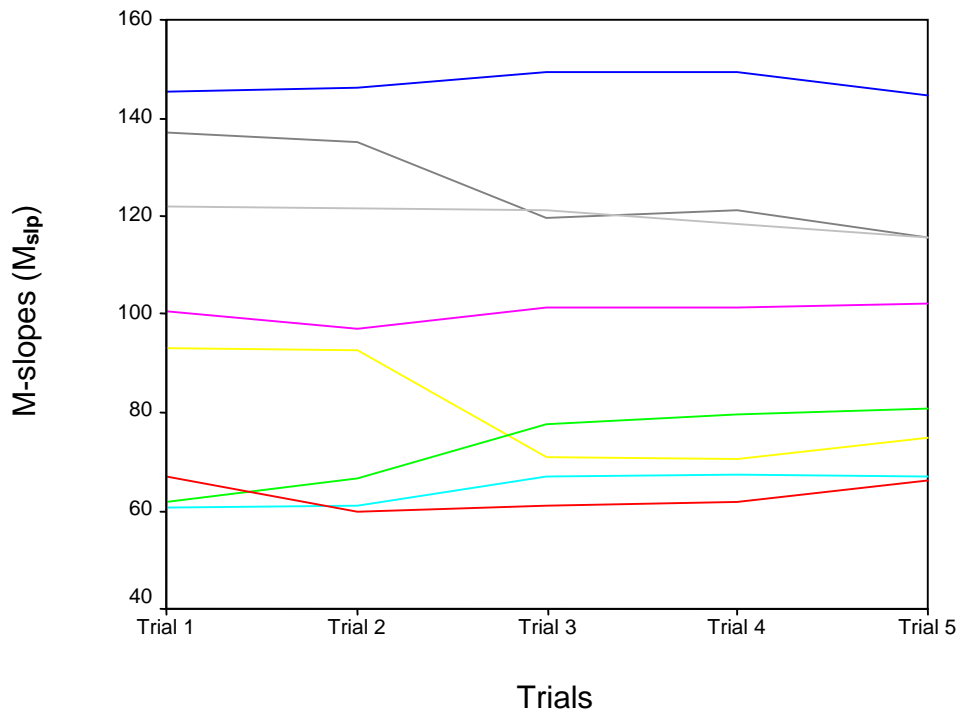

Fig.3.17C Changes in M-slopes in Trials 1-5 in sustained acupuncture (extended observation; $n=8$ ). One (1) subject was excluded by visual examination of M-slopes in Fig. 3.17B). Intraclass correlation coefficient was then improved from 0.9339 to 0.9581 , meeting the criteria for stability set for the study. 


\subsubsection{Analysis of $\mathbf{H}$-slopes Stability in Trials 1-2}

The ICC of the two H-slopes in Trials 1 and 2 of the 8 subjects was 0.9855 (Table 3.13), which indicated a high degree of consistency and stability of the $\alpha$-motoneuron pool prior to acupuncture. The means of the H-slopes in Trials 1 and 2 were also compared by using Paired-Sample T-Test. There was no significant difference between the Hslopes of the trials $(p=0.187)$. This also suggested insignificant difference in $\alpha$ motoneuron excitability between Trials 1 and 2 .

\subsubsection{Changes in Motoneuron Excitability}

\subsubsection{Changes in $\mathrm{H}_{\text {slp }} \underline{\text { after sustained acupuncture (extended observation) }}$}

The mean of the $\mathrm{H}_{\text {slp }}$ in Trials 1 and 2 was calculated and referred to as baseline. Individual changes in $\mathrm{H}_{\mathrm{slp}}$ during the experiment are shown in Fig. 3.18. The means and standard deviations of $\mathrm{H}_{\text {slp }}$ taken during the experiment were calculated and shown in Table 3.13. The large standard deviations reflected the marked inter-individual differences in baseline $\mathrm{H}_{\text {slp }}$ and individual subject's responses to acupuncture.

To determine significant differences in $\mathrm{H}_{\text {slp }}$ between baseline and Trials 3 to $5, \mathrm{H}_{\text {slp }}$ from individual subjects were pooled and analysed by repeated-measures ANOVA. The test showed a significant difference in $\mathrm{H}_{\text {slp }}$ between baseline and Trials 3 to 5 ( $p=$ 0.024). Between-subjects effect was also found to be significant $(p<0.0005)$. The results indicated that sustained acupuncture had resulted in significant changes in $\alpha$ motoneuron excitability between baseline and Trials 3 to 5 after acupuncture as assessed by $\mathrm{H}_{\text {slp }}$. A wide variation in individual responses to acupuncture was also demonstrated. 


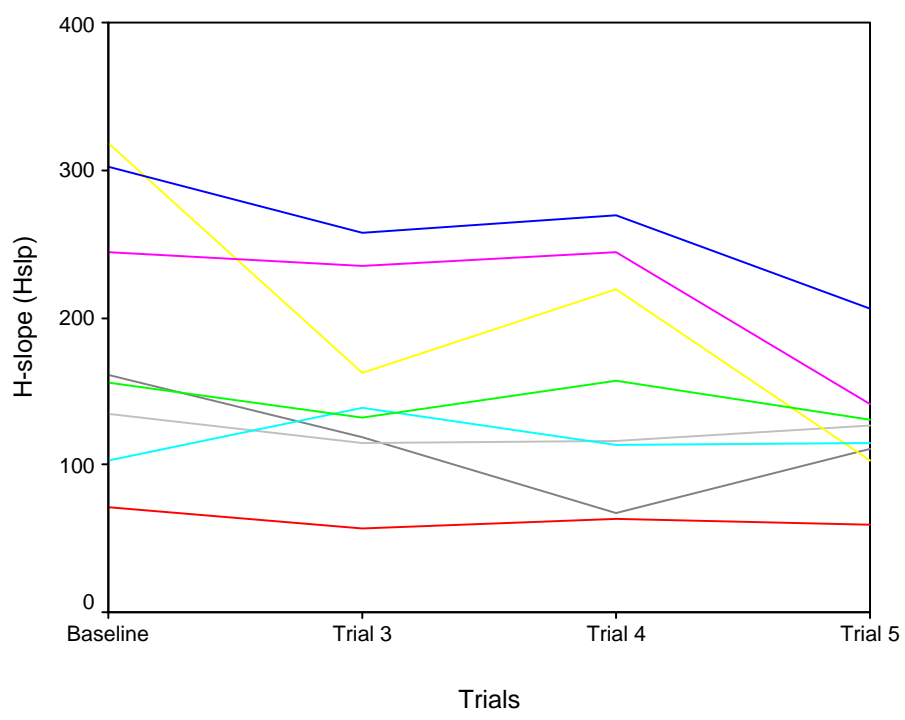

Fig. 3.18 Changes in $\mathrm{H}_{\text {slp }}$ (baseline, Trials 3-5) in sustained acupuncture (extended observation; $n=8)$.

Table 3.13 Descriptive statistics of $\mathrm{H}_{\text {slp }}$ (baseline, Trials 3-5) in sustained acupuncture (extended observation; $\mathrm{n}=8$ ).

\section{Descriptive Statistics}

\begin{tabular}{|l|r|r|r|r|r|}
\hline & $\mathrm{N}$ & Minimum & Maximum & \multicolumn{1}{c|}{ Mean } & Std. Deviation \\
\hline $\mathrm{H}_{\text {slp }}$ Baseline & 8 & 71.40 & 317.56 & 186.1389 & 91.55622 \\
$\mathrm{H}_{\text {slp Trial 3 }}$ & 8 & 56.57 & 257.66 & 151.9649 & 65.77372 \\
$\mathrm{H}_{\text {slp Trial 4 }}$ & 8 & 63.49 & 269.43 & 156.2284 & 79.51802 \\
$\mathrm{H}_{\text {slp }}$ Trial 5 & 8 & 58.90 & 206.04 & 123.9616 & 41.38065 \\
\hline
\end{tabular}




\subsubsection{Percentage Change in $\mathrm{H}_{\text {slp }}$ after sustained acupuncture (extended observation)}

Percentage changes in Hslp in Trials 3 to 5 after sustained acupuncture are shown in Fig. 3.19. Variations in individual responses to sustained acupuncture were apparent. The percentage change of $\mathrm{H}_{\text {slp }}$ in Trials 3 to 5 were pooled for statistical analysis (Table 3.14). The percentage change of $\mathrm{H}_{\text {slp }}$ in Trials 3 to 5 were subjected to One-Sample TTest analysis. The percentage changes of $\mathrm{H}_{\text {slp }}$ in Trial $3(p=0.147)$ and Trial $4(p=$ $0.108)$ were not significant but it was significant in Trial $5(p=0.023)$. This indicated that there was a significant change in $\alpha$-motoneuron excitability from baseline as measured by percentage change in $\mathrm{H}_{\text {slp }} 30$ minutes after completion of sustained acupuncture.

The percentage changes of $\mathrm{H}_{\text {slp }}$ in Trials 3-5 were also compared using repeated measures ANOVA. There was no significant difference between the three trials $(p=$ 0.240 ), which indicated the absence of significant change in motoneuron excitability between Trials 3 to 5 . This could be accounted for by the wide standard deviation in each trial. 


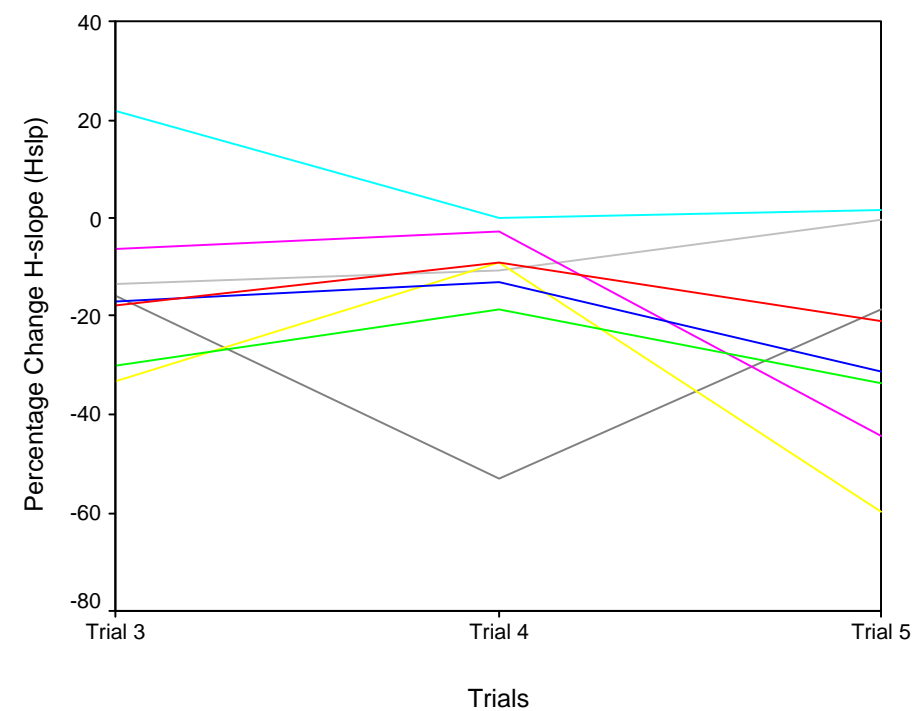

Fig. 3.19

Percentage changes in $\mathrm{H}_{\text {slp }}$ in Trials 3-5 after sustained acupuncture $(n=8)$.

Table 3.14 Descriptive statistics of percentage changes in $\mathrm{H}_{\text {slp }}$ in Trials 3-5 after sustained acupuncture $n=8$ ).

Descriptive Statistics

\begin{tabular}{|l|r|r|r|r|r|}
\hline & \multicolumn{1}{|c|}{$\mathrm{N}$} & Minimum & Maximum & \multicolumn{1}{c|}{ Mean } & Std. Deviation \\
\hline \%change $\mathrm{H}_{\text {slp }}$ Trial 3 & 8 & -48.93 & 34.83 & -13.6180 & 23.59924 \\
\%change $\mathrm{H}_{\text {slp }}$ Trial 4 & 8 & -58.12 & 10.81 & -14.1304 & 21.66441 \\
\%change $\mathrm{H}_{\text {slp }}$ Trial 5 & 8 & -67.64 & 12.50 & -24.9814 & 24.25429 \\
\hline
\end{tabular}




\subsubsection{Change in $\mathrm{H}_{\mathrm{slp}} \underline{\mathrm{M}}_{\mathrm{slp}}$ after sustained acupuncture (extended observation)}

The mean of $\mathrm{H}_{\mathrm{slp}} / \mathrm{M}_{\text {slp }}$ in Trials 1 and 2 was calculated and referred to as baseline. Individual changes in $\mathrm{H}_{\text {slp }} / \mathrm{M}_{\text {slp }}$ during the experiment are shown in Fig. 3.20. The means and standard deviations of $\mathrm{H}_{\mathrm{slp}} / \mathrm{M}_{\text {slp }}$ during the experiment were calculated and shown in Table 3.15. Inter-individual differences in baseline $H_{\text {slp }} / M_{\text {slp }}$ and responses to acupuncture were evident by the large standard deviation in comparison to the mean.

To determine significant differences in $\mathrm{H}_{\text {slp }} / \mathrm{M}_{\text {slp }}$ between baseline and Trials 3 to 5 , $\mathrm{H}_{\mathrm{slp}} / \mathrm{M}_{\text {slp }}$ from individual subjects were pooled and analysed by repeated-measures ANOVA. There was significant difference in $\mathrm{H}_{\mathrm{slp}} / \mathrm{M}_{\text {slp }}$ between the baseline and Trials 3 to $5(p=0.017)$. Between-subjects effect was found to be significant $(p<0.0005)$. The results indicated that sustained acupuncture had resulted in significant changes in $\alpha$-motoneuron excitability between baseline and Trials 3 to 5 after acupuncture as assessed by comparison of $\mathrm{H}_{\mathrm{slp}} / \mathrm{M}_{\mathrm{slp}}$. A wide variation in individual responses to acupuncture was also demonstrated. 


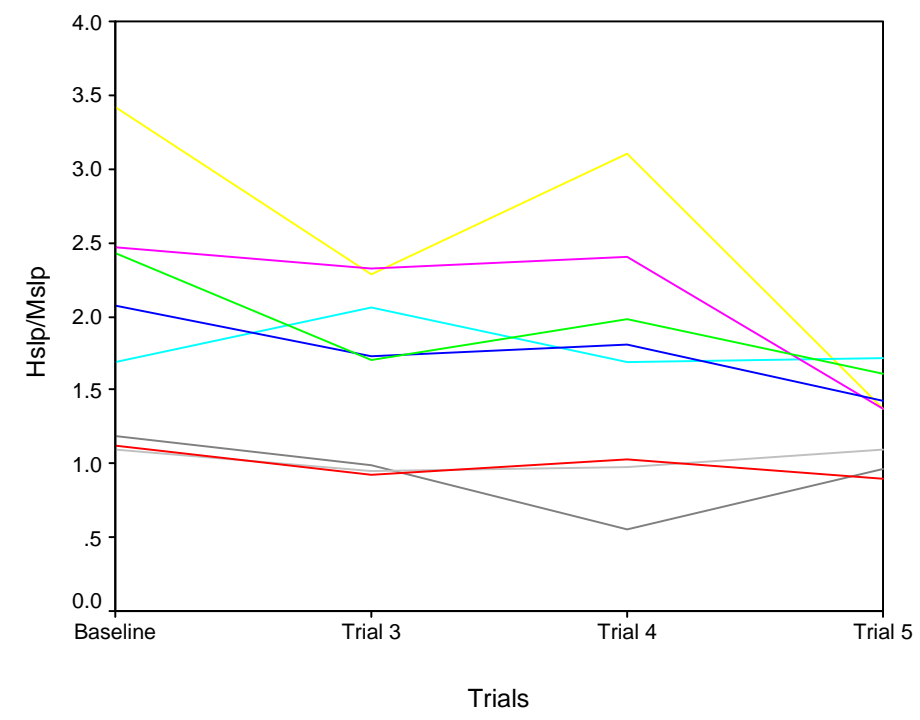

Fig. 3.20 Changes in $\mathrm{H}_{\text {slp }} / \mathrm{M}_{\text {slp }}$ (baseline, Trials 3-5) in sustained acupuncture (extended observation; $\mathrm{n}=8$ ).

Table 3.15 Descriptive statistics of $\mathrm{H}_{\text {slp }} / \mathrm{M}_{\text {slp }}$ (baseline, Trials 3-5) in sustained acupuncture (extended observation; $\mathrm{n}=8$ ).

\section{Descriptive Statistics}

\begin{tabular}{|l|r|r|r|r|r|}
\hline & $\mathrm{N}$ & Minimum & Maximum & \multicolumn{1}{c|}{ Mean } & Std. Deviation \\
\hline $\mathrm{H}_{\text {slp }} / \mathrm{M}_{\text {slp }}$ Baseline & 8 & 1.10 & 3.42 & 1.9366 & .82109 \\
$\mathrm{H}_{\text {slp }} / \mathrm{M}_{\text {slp }}$ Trial 3 & 8 & .93 & 2.32 & 1.6197 & .59182 \\
$\mathrm{H}_{\text {slp }} / \mathrm{M}_{\text {slp }}$ Trial 4 & 8 & .56 & 3.10 & 1.6934 & .83035 \\
$\mathrm{H}_{\text {slp }} / \mathrm{M}_{\text {slp }}$ Trial 5 & 8 & .89 & 1.72 & 1.3080 & .29917 \\
\hline
\end{tabular}




\subsubsection{Percentage Change in $\mathrm{H}_{s l p} \underline{\mathrm{M}_{s l p}}$ after sustained acupuncture (extended}

observation)

Percentage changes in $\mathrm{H}_{\text {slp }} / \mathrm{M}_{\text {slp }}$ in Trials 3 to 5 after sustained acupuncture are shown in Fig. 3.21. Variations in individual responses to sustained acupuncture were still apparent. The percentage change of $\mathrm{H}_{\mathrm{slp}} / \mathrm{M}_{\mathrm{slp}}$ in Trials 3 to 5 were pooled for statistical analysis (Table 3.16). There appeared to be a negative percentage change in $\mathrm{H}_{\text {slp }} / \mathrm{M}_{\text {slp }}$ immediately and 15 minutes after sustained acupuncture followed by a further reduction 30 minutes later. The percentage changes of $\mathrm{H}_{\text {slp }} / \mathrm{M}_{\text {slp }}$ in Trials 3 to 5 were subjected to One-Sample T-Test analysis. The percentage change of $\mathrm{H}_{\text {slp }} / \mathrm{M}_{\text {slp }}$ in Trial $3(p=0.053)$ was not significant, but those in Trial $4(p=0.043)$, and Trial $5(p=0.010)$ were significant. This indicated that there was significant difference in $\alpha$-motoneuron excitability from baseline measured by percentage change in $\mathrm{H}_{\mathrm{slp}} / \mathrm{M}_{\mathrm{slp}}$ as a result of sustained acupuncture. The effect was significant at 15 minutes and remained significant up to 30 minutes after withdrawal of the needles. The percentage change of $\mathrm{H}_{\text {slp }} / \mathrm{M}_{\text {slp }}$ in Trials 3 to 5 were also compared using repeated measures ANOVA. There was no significant difference between the three trials $(p=0.243)$, which indicated the absence of significant change in motoneuron excitability between Trials 3 to 5 . This could be accounted for by the wide standard deviation in each trial. 


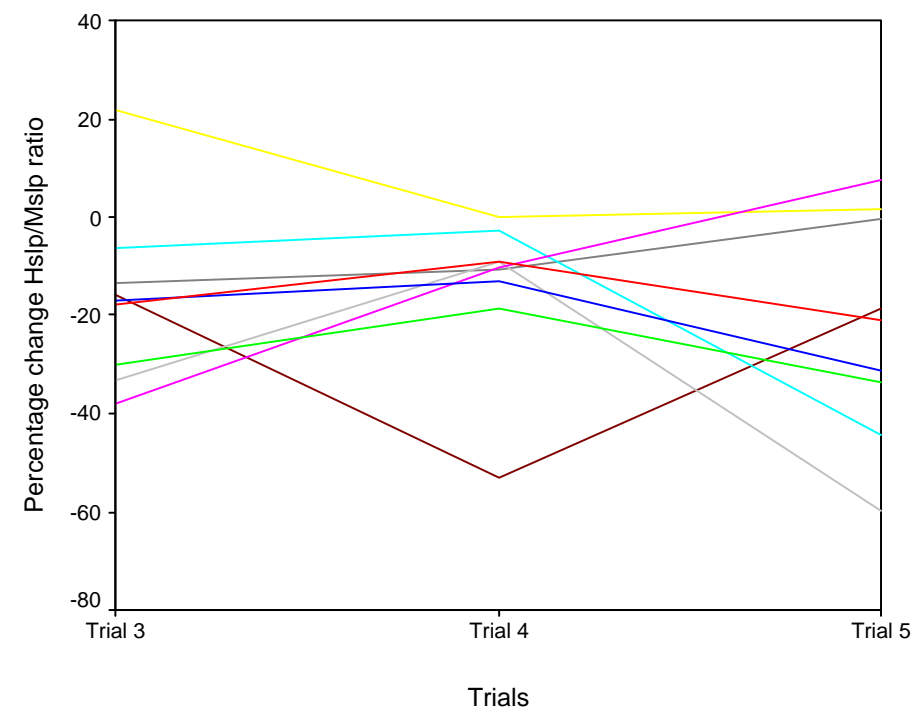

Fig. 3.21 Percentage changes in $\mathrm{H}_{\text {slp }} / \mathrm{M}_{\text {slp }}$ in Trials 3-5 after sustained acupuncture (extended observation; $n=8)$.

Table 3.16 Descriptive statistics of percentage changes in $\mathrm{H}_{\text {slp }} / \mathrm{M}_{\text {slp }}$ in Trials 3-5 after sustained acupuncture (extended observation; $n=8$ ).

\section{Descriptive Statistics}

\begin{tabular}{|l|r|r|r|r|r|}
\hline & $\mathrm{N}$ & Minimum & Maximum & \multicolumn{1}{c|}{ Mean } & Std. Deviation \\
\hline \%change $\mathrm{H}_{\text {sIp }} / \mathrm{M}_{\text {slp }}$ Trial 3 & 8 & -33.20 & 21.91 & -13.9441 & 16.92160 \\
\%change $\mathrm{H}_{\text {slp }} / \mathrm{M}_{\text {slp }}$ Trial 4 & 8 & -52.97 & .14 & -14.5027 & 16.57431 \\
\%change $\mathrm{H}_{\text {slp }} / \mathrm{M}_{\text {slp }}$ Trial 5 & 8 & -59.71 & 1.77 & -25.8772 & 20.92022 \\
\hline
\end{tabular}




\subsubsection{Conclusion}

After sustained acupuncture, there was a significant change in $\alpha$-motoneuron excitability when assessed by changes in $\mathrm{H}_{\text {slp }}$ and $\mathrm{H}_{\text {slp }} / \mathrm{M}_{\mathrm{slp}}$ up to 30 minutes after withdrawal of the needles. Percentage changes in $\mathrm{H}_{\mathrm{slp}} / \mathrm{M}_{\text {slp }}$ demonstrated that significant changes occurred from 15 minutes after completion of sustained acupuncture and the effects were still apparent 30 minutes following the intervention. A wide variation in individual responses to sustained acupuncture was observed. 


\subsection{Comparison of effects of short duration and sustained acupuncture on motoneuron excitability}

The duration of acupuncture needling is one of the factors affecting the total amount of stimulation (Campbell, 1998). In general, acupuncture needles are retained for 15 to 20 minutes during treatment (Cheng, 1987; Helms, 1995). However, clinically effective needling duration as brief as 1-2 minutes has been reported (Campbell, 1999).

To determine the differential effects of short duration and sustained acupuncture on $\alpha$ motoneuron excitability, data from 13 subjects included in final analyses of both short duration and sustained acupuncture studies were grouped for further analysis. Changes in $H_{\text {slp }}$ and $H_{\text {slp }} / M_{\text {slp }}$ from baseline and percentage changes in $H_{\text {slp }}$ and $H_{\text {slp }} / M_{\text {slp }}$ after acupuncture were subjected to analysis by repeated measures ANOVA, with duration of acupuncture and interval of assessment as factors. No significant differences were found in these parameters between short duration and sustained acupuncture (Table 3.17 and Fig. 3.22-3.25). There was also no significant interaction between the duration of acupuncture and the interval of assessment.

This indicated that there was no significant difference in the effect on $\alpha$-motoneuron excitability between short duration and sustained acupuncture. 
Table 3.17 Results of repeated measures ANOVA comparing different parameters of $\alpha$ motoneuron excitability in short duration and sustained acupuncture.

\begin{tabular}{|c|c|c|c|c|}
\hline & Changes in $\mathrm{H}_{\text {slp }}$ & \%change in $\mathrm{H}_{\text {slp }}$ & Changes in $\mathbf{H}_{\text {slp }} / \mathbf{M}_{\text {slp }}$ & \%change $\mathbf{H}_{\text {slp }} / \mathbf{M}_{\text {slp }}$ \\
\hline $\begin{array}{c}\text { Effect of Duration of } \\
\text { Acupuncture }\end{array}$ & $p=0.675$ & $p=0.178$ & $p=0.495$ & $p=0.277$ \\
\hline $\begin{array}{c}\text { Effect of Interval of } \\
\text { Assessment }\end{array}$ & $p=0.099$ & $p=0.187$ & $p=0.081$ & $p=0.335$ \\
\hline $\begin{array}{c}\text { Interaction of } \\
\text { Duration*Interval }\end{array}$ & $p=0.385$ & $p=0.846$ & $p=0.501$ & $p=0.810$ \\
\hline
\end{tabular}




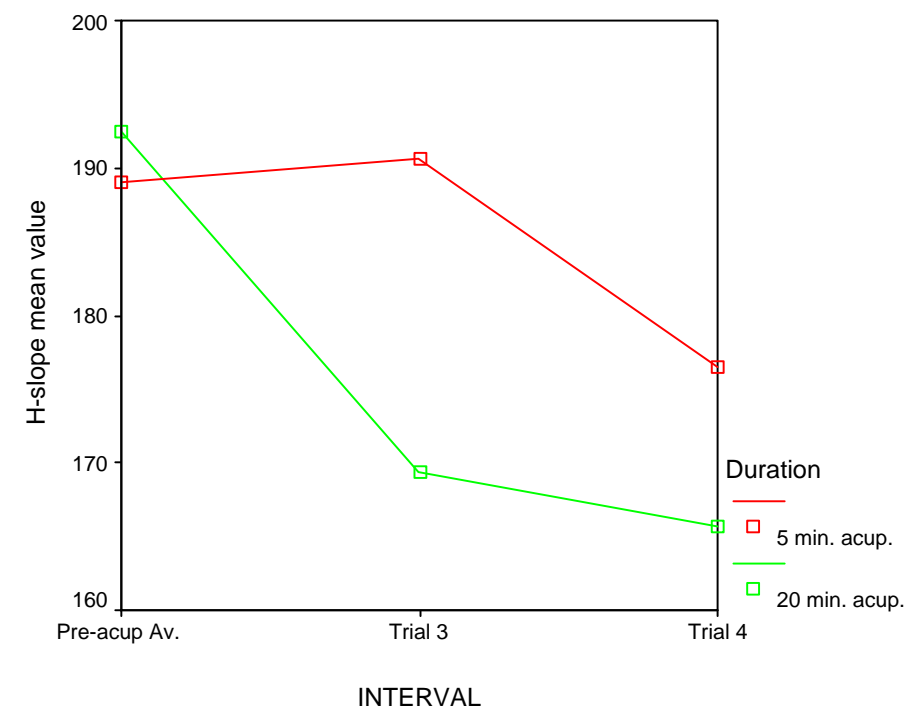

Fig. 3.22

Relative changes in the means of $\mathrm{H}_{\text {sip }}$ before and after short duration and sustained acupuncture $(n=13)$. The effect of duration of acupuncture $(p=0.675)$ and interval of assessment $(p=0.099)$ were not significant.

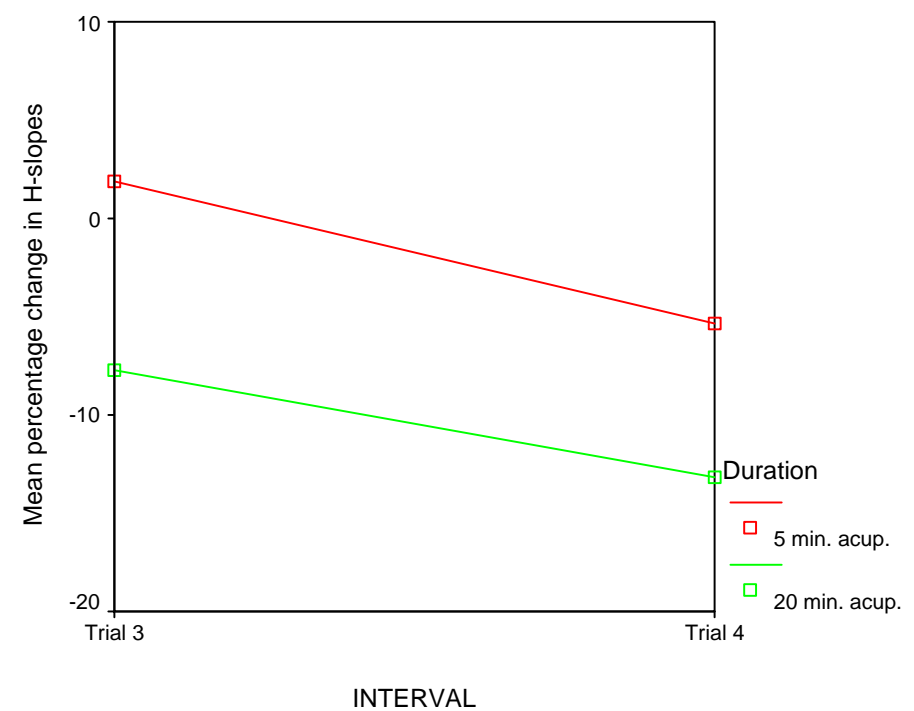

Fig. 3.23

Relative percentage changes in $\mathrm{H}_{\text {slp }}$ in Trials 3-4 after short duration and sustained acupuncture $(n=13)$. The difference was not significant with respect to duration of acupuncture $(p=0.178)$ or interval of assessment $(p=0.187)$ 


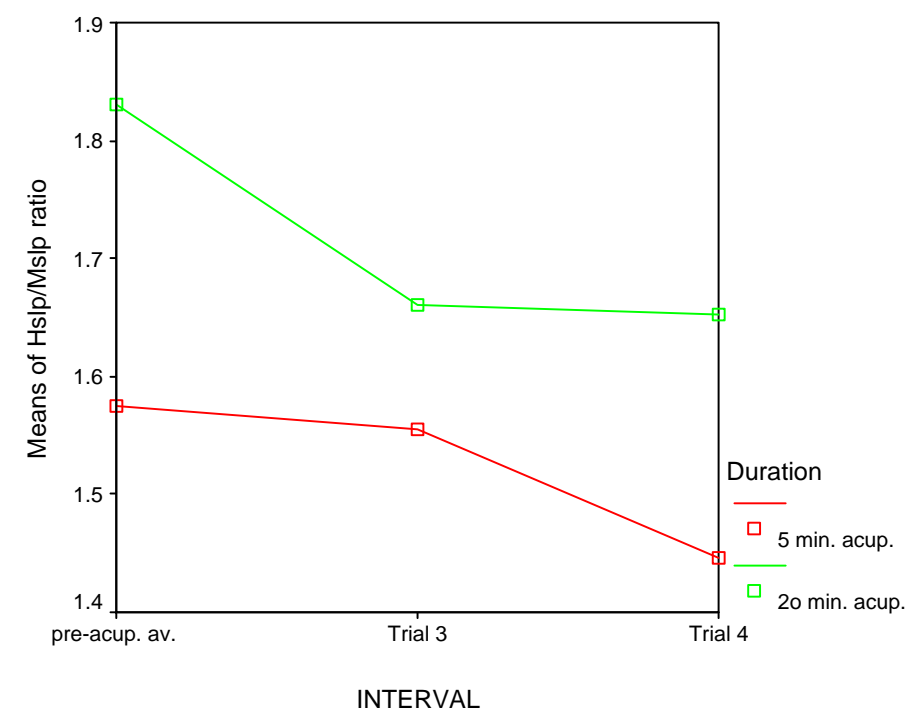

Fig. 3.24

Relative changes in $\mathrm{H}_{\text {sip }} / \mathrm{M}_{\text {slp }}$ before and after short duration and sustained acupuncture $(n=13)$. The difference was not significant with respect to duration of acupuncture $(p=0.495)$ or interval of assessment $(p=0.081)$.

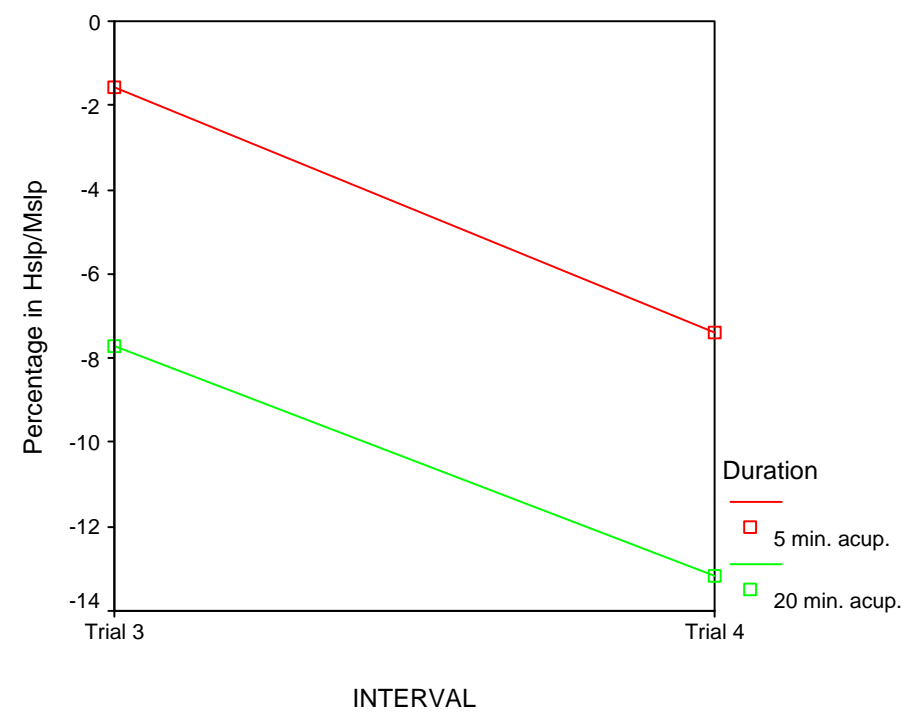

Fig. 3.25

Relative percentage changes in $\mathrm{H}_{\text {sip }} / \mathrm{M}_{\text {slp }}$ in Trials 3-4 after short duration and sustained acupuncture $(n=13)$. The difference was not significant with respect to duration of acupuncture $(p=0.227)$ or interval of assessment $(p=0.335)$. 


\subsection{Effect of intensity of acupuncture sensation on $\alpha$-motoneuron excitability}

According to traditional acupuncture techniques, the presence of acupuncture sensation (deqi) is a pre-requisite for effective needling (Cheng, 1987; Helms, 1995). The relationship between the perceived intensity of acupuncture sensation (deqi) and changes in motoneuron excitability in this study was explored using Spearman's Rank Order Correlation. There was no significant correlation between the perceived intensity of acupuncture sensation (deqi) and percentage changes in $\mathrm{H}_{\text {slp }}$ or $\mathrm{H}_{\mathrm{slp}} / \mathrm{M}_{\text {slp }}$ after short duration (Table 3.18) or sustained acupuncture (Table 3.19).

This indicated that the intensity of perceived acupuncture sensation (deqi) has no significant correlation to the changes in $\alpha$-motoneuron excitability as a result of acupuncture. 
Table 3.18 Correlation between Intensity of Acupuncture Sensation and Changes in H-reflex (Short Duration Acupuncture)

\begin{tabular}{|c|c|c|c|c|c|}
\hline & & $\begin{array}{c}\% \text { change of } \\
\text { Hslp Trial } 3\end{array}$ & $\begin{array}{c}\% \text { change of } \\
\text { Hslp Trial } 4\end{array}$ & $\begin{array}{c}\% \text { change of } \\
\text { Hslp/Mslp Trial } 3\end{array}$ & $\begin{array}{c}\text { \% change of } \\
\text { Hslp/Mslp Trial } 4\end{array}$ \\
\hline \multirow[t]{2}{*}{$\begin{array}{l}\text { Intensity of } \\
\text { Acupuncture } \\
\text { Sensation }\end{array}$} & $\begin{array}{l}\text { Correlation } \\
\text { Coefficient }\end{array}$ & -0.006 & -0.128 & -0.065 & -0.201 \\
\hline & Sig. (2-tailed) & 0.983 & 0.637 & 0.810 & 0.456 \\
\hline
\end{tabular}

Table 3.19 Correlation between Intensity of Acupuncture Sensation and Changes in H-reflex (Sustained Acupuncture)

\begin{tabular}{|c|c|c|c|c|c|}
\hline & & $\begin{array}{c}\% \text { change of } \\
\text { Hslp Trial } 3\end{array}$ & $\begin{array}{c}\% \text { change of } \\
\text { Hslp Trial } 4\end{array}$ & $\begin{array}{c}\text { \% change of } \\
\text { Hslp/Mslp Trial } 3\end{array}$ & $\begin{array}{c}\text { \% change of } \\
\text { Hslp/Mslp Trial } 4\end{array}$ \\
\hline \multirow{2}{*}{$\begin{array}{l}\text { Intensity of } \\
\text { Acupuncture } \\
\text { Sensation }\end{array}$} & $\begin{array}{c}\text { Correlation } \\
\text { Coefficient }\end{array}$ & -0.377 & -0.380 & -0.495 & -0.470 \\
\hline & Sig. (2-tailed) & 0.166 & 0.162 & 0.061 & 0.077 \\
\hline
\end{tabular}




\subsection{Summary of Results}

Tab. 3.20 Summary of results in short duration and sustained acupuncture studies

\begin{tabular}{|c|c|c|c|c|}
\hline & $\begin{array}{l}\text { Short Duration Acupuncture } \\
\qquad(\mathrm{n}=16)\end{array}$ & $\begin{array}{l}\text { Short Duration Acupuncture } \\
\text { (extended trial) } \\
(\mathrm{n}=8)\end{array}$ & $\begin{array}{l}\text { Sustained Acupuncture } \\
\qquad(n=15)\end{array}$ & $\begin{array}{l}\text { Sustained Acupuncture } \\
\text { (extended trial) } \\
(\mathrm{n}=8)\end{array}$ \\
\hline $\begin{array}{l}\text { All trials } \mathrm{M}_{\mathrm{slp}} \text { stability } \\
\text { (Intraclass correlation coefficient) }\end{array}$ & 0.9740 & 0.9717 & 0.9635 & 0.9581 \\
\hline $\begin{array}{l}\text { Pre-acupuncture } \mathrm{H}_{\mathrm{slp}} \text { stability } \\
\text { (Intraclass correlation coefficient) }\end{array}$ & 0.9784 & 0.9728 & 0.9609 & 0.9863 \\
\hline Changes in $\mathrm{H}_{\mathrm{sip}}$ pre- and post-acupuncture & $\begin{array}{l}\text { Not significant } \\
\qquad(p=0.484)\end{array}$ & $\begin{array}{l}\text { Not significant } \\
\qquad(p=0.834) .\end{array}$ & $\begin{array}{l}\text { Significant } \\
(p=0.038)^{*}\end{array}$ & $\begin{array}{l}\text { Significant } \\
(p=0.024)^{*}\end{array}$ \\
\hline Percentage changes in $\mathrm{H}_{\text {slp }}$ post-acupuncture & $\begin{array}{c}\text { Not significant } \\
\text { (trial } 3, p=0.844 \text { ) } \\
\text { (trial } 4, p=0.248 \text { ) }\end{array}$ & $\begin{array}{c}\text { Not significant } \\
\text { (trial } 3, p=0.719 \text { ) } \\
\text { (trial } 4, p=0.355 \text { ) } \\
\text { (trial } 5, p=0.478 \text { ) }\end{array}$ & $\begin{array}{c}\text { Not significant } \\
\text { (trial } 3, p=0.134 \text { ) } \\
\text { Significant } \\
\text { (trial } 4, p=0.015 \text { )* }\end{array}$ & 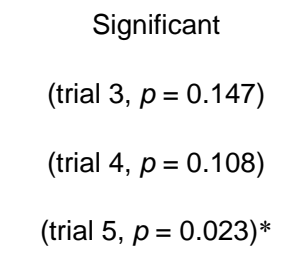 \\
\hline Changes in $\mathrm{H}_{\text {slp }} / \mathrm{M}_{\mathrm{slp}}$ pre- and post-acupuncture & $\begin{array}{l}\text { Not significant } \\
\qquad(p=0.157)\end{array}$ & $\begin{array}{l}\text { Not significant } \\
\qquad(p=0.608)\end{array}$ & $\begin{array}{l}\text { Significant } \\
(p=0.006)^{*}\end{array}$ & $\begin{array}{l}\text { Significant } \\
(p=0.017)^{*}\end{array}$ \\
\hline $\begin{array}{l}\text { Percentage changes in } \mathrm{H}_{\text {slp }} / \mathrm{M}_{\text {slp }} \text { post- } \\
\text { acupuncture }\end{array}$ & $\begin{array}{c}\text { Not significant } \\
\text { (trial } 3, p=0.438 \text { ) } \\
\text { (trial } 4, p=0.168 \text { ) }\end{array}$ & $\begin{array}{c}\text { Not significant } \\
\text { (trial } 3, p=0.693 \text { ) } \\
\text { (trial 4, } p=0.215 \text { ) } \\
\text { (trial } 5, p=0.233 \text { ) }\end{array}$ & $\begin{array}{c}\text { Not significant } \\
\text { (trial } 3, p=0.069 \text { ) } \\
\text { Significant } \\
\text { (trial } 4, p=0.004 \text { )* }\end{array}$ & $\begin{array}{c}\text { Significant } \\
\text { (trial } 3, p=0.053 \text { ) } \\
\text { (trial } 4, p=0.043 \text { )* } \\
\text { (trial } 5, p=0.010 \text { )* }\end{array}$ \\
\hline
\end{tabular}


Tab. 3.21

excitability $(n=13)$

\begin{tabular}{|l|c|c|}
\hline \multicolumn{1}{|c|}{ Parameters used for assessment } & $\begin{array}{c}\text { Effect of Duration of Acupuncture } \\
\text { (short duration vs sustained) }\end{array}$ & $\begin{array}{c}\text { Effect of Time Interval after } \\
\text { Acupuncture } \\
\text { (Trial 3 vs Trial 4) }\end{array}$ \\
\hline Difference in $\mathrm{H}_{\mathrm{slp}}$ before and after acupuncture & Not significant $(p=0.675)$ & Not significant $(p=0.099)$ \\
\hline Percentage change in $\mathrm{H}_{\mathrm{slp}}$ in Trials 3 \& 4 & Not significant $(p=0.178)$ & Not significant $(p=0.187)$ \\
\hline Difference in $\mathrm{H}_{\mathrm{slp}} / \mathrm{M}_{\mathrm{slp}}$ before and after acupuncture & Not significant $(p=0.495)$ & Not significant $(p=0.081)$ \\
\hline Percentage change in $\mathrm{H}_{\mathrm{sl}} / \mathrm{M}_{\mathrm{slp}}$ in Trials 3 \& 4 & Not significant $(p=0.277)$ & Not significant $(p=0.335)$ \\
\hline
\end{tabular}




\section{Chapter 4 Discussion}

\subsection{Major findings}

The most significant findings from the present study were that short duration manual acupuncture had no effect on the excitability of the $\alpha$-motoneuron pool when measured using the H-reflex. On the other hand, sustained manual acupuncture caused a marked decrease in $\alpha$-motoneuron excitability. The effect was statistically significant at 15 minutes and was maintained for at least 30 minutes after withdrawal of needles.

Further, the intensity of sensation produced by acupuncture (deqi) was not related to the inhibitory effect.

Other findings include the following:

- Although the changes did not reach statistical significance, the inhibition of $\alpha$ motoneuron excitability after short duration acupuncture increased over time.

- The lack of significant statistical difference between the effects of short duration and sustained acupuncture on $\alpha$-motoneuron excitability also indicated that short duration acupuncture might have influenced $\alpha$-motoneuron excitability.

- The use of the parameter $\mathrm{H}_{\text {slp }} / \mathrm{M}_{\text {slp }}$ was more sensitive for assessing changes in $\alpha$ motoneuron excitability than $\mathrm{H}_{\text {slp }}$. 


\subsection{Acupuncture and $\alpha$-Motoneuron Excitability}

The effect of acupuncture on $\alpha$-motoneuron excitability in normal subjects has been studied by various authors using measurement of the stretch reflex or the H-reflex (Chang et al., 2001; Dawson, Lippold, \& Milne, 1987; Milne et al., 1985). This study confirmed previous work that found acupuncture to have statistically insignificant effect on the excitability of the motoneuron pool immediately after the intervention (Chang et al., 2001; Dawson et al., 1987; Milne et al., 1985). While Milne et al. (Milne et al., 1985) and Dawson et al. (Dawson et al., 1987) observed a transient inhibition during acupuncture, Chang et al. (2001) found no change in the soleus H-reflex during and following acupuncture to the contralateral hand. In this study, however, acupuncture delivered over a longer time frame according to usual clinical practice, had a significant inhibitory effect which persisted after removal of the needles. The inhibitory effect of acupuncture on $\alpha$-motoneuron excitability demonstrated in this study could be related to a different experimental design, specifically the inclusion of an extended period of observation, sustained acupuncture stimulation, measurement of H-reflex in the same limb, and the use of different parameters in the analysis. These differences that may explain the discrepancies of findings in this current study from previous work will be discussed in further details in the sections below.

\subsubsection{Effects of sites of acupuncture and H-reflex recordings}

In previous studies, manual acupuncture (Chang et al., 2001) or electroacupuncture was administered to an upper limb (Dawson et al., 1987; Milne et al., 1985). Recordings were then made in the contralateral upper (Dawson et al., 1987; Milne et al., 1985) or lower limb (Chang et al., 2001). The measured effects of these interventions were expected to be mainly extrasegmental in origin. In this study, manual acupuncture was 
applied to and H-reflex recordings were recorded from the same leg. Therefore, the combination of spinal segmental and supraspinal effects of acupuncture on $\alpha$ motoneuron excitability was measured in this study. It is possible that these effects are synergistic and thus greater than the supraspinal effect alone on the pool of $\alpha$ motoneurons being measured (Pomeranz, 2001).

\subsubsection{Effect of timing the H-reflex recordings}

Timing of the post-intervention H-reflex recording could also be an important factor in unraveling the total effects of acupuncture on $\alpha$-motoneuron excitability. In this study, the periods of observation were spaced differently to those in the Chang et al. study (Chang et al., 2001). This allowed for delayed humoral effects from acupuncture which may appear 30 minutes after the removal of needles (Sjolund et al., 1977). Chang et al. (2001) observed the H-reflexes up to 25 minutes from the beginning of acupuncture. In this study, significant change was only identified at 35 minutes after insertion of needles in the sustained acupuncture series. This shorter period of observation by Chang et al. (2001) might have resulted in missing the experimental effect.

\subsubsection{Effect of amount of afferent input from acupuncture}

The total amount of afferent input delivered by an intervention may affect the amount of physiological response from the body (Leshnower, Potts, Garry, \& Mitchell, 2001). This has been demonstrated with electroacupuncture in rats in which an increase in analgesia occurred with increase in stimulation intensity at the same frequency and with the same acupuncture points (Huang et al., 2002; Romita, Suk, \& Henry, 1997). Higher intensity of transcutaneous electrical stimulation of acupuncture points was also shown to result in a greater reduction in post-operative analgesia than low intensity stimulation 
(Wang et al., 1997). Even if this effect was due to higher threshold receptors being stimulated, the finding is still a function of the increased amount of afferent input delivered. During sustained acupuncture in the present study, two acupuncture points were stimulated for 20 minutes. This was in contrast to the Chang et al. study (2001) where stimulation was only given to a single acupuncture point for 15 minutes. A greater amount of afferent input would result in summation at the spinal cord and a greater response from $\alpha$-motoneurons.

\subsubsection{Effect of manual acupuncture versus electroacupuncture}

The use of manual acupuncture in this study could also have influenced the results. Dawson et al. (1987) found the inhibitory effect on the reflex response to muscle stretch with electroacupuncture began to fade during the intervention although it was still demonstrable up to 30 minutes post-intervention. Hsieh et al.(2002), however, did not find any effect of $2 \mathrm{~Hz}$ electroacupuncture at the acupuncture point Zusanli (ST36) on H-reflex in healthy subjects. These results suggest that different neural pathways and brain mechanisms could be involved in electroacupuncture and manual acupuncture.

In support of this proposition, a recent functional magnetic resonance imaging (fMRI) study has demonstrated that manual and electroacupuncture affected different parts of the brain (Kong et al., 2002). Electroacupuncture mainly produced signal increases in precentral gyrus, postcentral gyrus/inferior parietal lobule, and putamen/insula; in contrast, manual needle manipulation produced prominent decreases of fMRI signals in posterior cingulate, superior temporal gyrus, and putamen/insula (Kong et al., 2002). Electroacupuncture also increased the concentrations of calcitonin gene-related peptide (CGRP) in cerebrospinal fluid and the frontal cortex of the brain in rats while manual acupuncture had no effect (Wyon, Hammar, Theodorsson, \& Lundeberg, 1998). 
Experiments performed in rats using electroacupuncture demonstrated increased levels of substance P- (SP), neuropeptide Y- (NPY), and neurokinin A (NKA)-like immunoreactivity (-LI) in the hippocampus, and NPY-LI in the occipital cortex, while manual acupuncture did not evoke change in any one of the neuropeptides tested (Bucinskaite et al., 1994). The different physiological responses of the body to manual compared to electroacupuncture may, therefore, reflect in the differential responses of $\alpha$-motoneuron excitability after the respective intervention.

\subsubsection{Effect of different H-reflex methodology}

The use of a different H-reflex methodology for assessing $\alpha$-motoneuron pool excitability could also contribute to different findings. In this study, a range of stimulation intensities was used to elicit the H-reflexes from which H-reflex recruitment curves were constructed. The rate of change of the ascending part of the recruitment curve was then measured by calculating $\mathrm{H}_{\text {slp }}$, and $\mathrm{H}_{\text {slp }} / \mathrm{M}_{\text {slp }}$. In previous studies (Chang et al., 2001; Dawson et al., 1987; Milne et al., 1985), the change in magnitude of the Hreflex in response to a single electrical stimulation intensity to the peripheral nerve was used to assess $\alpha$-motoneuron pool excitability. A single stimulation intensity would be expected to evoke responses from a smaller pool of $\alpha$-motoneurons in the spinal cord. In this study, however, the rate of change in motoneuron responses to a range of stimulation intensities reflected the responses from a much wider pool of $\alpha$ motoneurons from small to large according to the "size principle" (Henneman et al., 1965). This technique would, therefore, be more sensitive to changes in the spinal cord than experiments using a single stimulation intensity.

Use of different parameters for measuring change in motoneuron excitability could also account for the lack of significant effect revealed in other studies. For example, the use 
of the greatest $\mathrm{H} / \mathrm{M}$ ratio parameter by Hsieh et al. (2002) demonstrated no significant change 30 minutes after electroacupuncture in the ipsilateral or contralateral leg. It may be that the $\mathrm{H}_{\max } / \mathrm{M}_{\max }$ ratio is less sensitive in identifying changes in the H-reflex than the $H_{\text {slp }}$ and $H_{\text {slp }} / M_{\text {slp }}$ parameters (Bradnam et al., 2000; Funase et al., 1996).

In summary, the use of a range of stimulation intensities to elicit responses from a wider pool of motoneurons and measurement of the change in slopes of the H-reflex and Mwave recruitment curves has proved more sensitive in evaluating change in the $\alpha$ motoneuron excitability than previous studies. This may be the reason that a significant inhibitory effect on $\alpha$-motoneuron excitability was revealed in this study and not in previous work.

\subsubsection{Effect of specificity of acupuncture points}

Different acupuncture points are purposely used in clinical situations to elicit different therapeutic effects (Cheng, 1987; Helms, 1995). It has been shown by fMRI that different areas of the brain are activated by stimulation of specific acupuncture points. For example, BL 67 (Zhiyin), BL 65 (Shugu), and BL 60 (Kunlun) activated the primary visual cortex, while the adjacent BL 66 (Zutonggu) along the same meridian did not (Cho et al., 1998); and stimulation of ST 36 (Zusanli) activated the hippocampus while GB 34 (Yanglingquan) activated the hypothalamus, insula and the motor cortex (Chiu et al., 2001). LI 4 (Hoku) and LI 11 (Quchi) were the acupuncture points used in the other studies on healthy subjects (Chang et al., 2001; N.J. Dawson et al., 1987; Milne et al., 1985), while SP 9 (Yinlingquan) and GB 34 (Yanglingquan) were used in this study. The different acupuncture points used might have resulted in different effects 
on the central nervous system. Further studies on the specificity of acupuncture points will be required to clarify the picture.

\subsubsection{Difference between healthy subjects and stroke patients}

The results of the present study concurred with that of a study investigating changes in a-motoneuron excitability in stroke patients (Yu et al., 1995), in which significantly increased H-reflex recovery time was found after acupuncture indicating a decrease in motoneuron excitability. However, in the Yu et al study (1995) the onset of the inhibitory effect occurred immediately after acupuncture rather than as a delayed response as found in this current work. This could be related to the increased afferent input from use of five acupuncture points as opposed to two used in the present study.

A higher baseline $\alpha$-motoneuron excitability in stroke patients could also contribute to the difference in response. The effects of acupuncture can depend significantly on the variation of the baseline condition from normal. In a group of subjects with asymmetrical electromyogram (EMG) readings in the two sides of the lumbar paraspinal muscles, it was shown that in those subjects whose baseline EMG readings were high compared to the median of the group, the absolute EMG values significantly decreased after acupuncture, while in subjects with low baseline reading compared to the median, acupuncture increased the absolute EMG values (Tanaka et al., 1998). Acupuncture is also known to lower blood pressure in hypertensives, raise blood pressure in hypotensives, and increase urinary sodium excretion during hyperosmotic challenge (Yao, 1993). Acupuncture is generally identified as a form of regulatory therapy which encourages a change towards homeostasis (Helms, 1995). Thus, in subjects who have near normal baseline levels, like the normal subjects used in this 
study, the changes after acupuncture may not be as marked as in subjects with pathological conditions. That the affected side of the stroke patients had heightened $\alpha-$ motoneuron excitability was demonstrated by a shorter H-reflex recovery time compared to the unaffected side and to normal controls (Yu et al., 1995). This suggests that the affected side of stroke subjects had a higher baseline $\alpha$-motoneuron excitability. The earlier appearance of significant reduction in $\alpha$-motoneuron excitability in stroke patients 20 minutes after insertion of needle as compared to 35 minutes in this study could be partially explained by a greater homeostatic response in the stroke patients whose $\alpha$-motoneuron excitability had a greater deviation from the normal baseline.

\subsubsection{Variation of individual responses after acupuncture}

In this study there was considerable individual variability in the change in $\alpha$ motoneuron excitability although the overall statistical trend was one of inhibition. This finding was in accordance with the experience of acupuncture practitioners who recognize that patients exhibit different degrees of response to this form of therapy (Baldry, 1998; Campbell, 1998; Mann, 1992). This has been described in terms of "strong reactors" and "normal reactors" to reflect clinical observation of at least two different groups according to their rates of response (Mann, 1992). In addition, Campbell (1998) has labeled a group as "non-responders" as those who show no therapeutic response to acupuncture. A similar phenomenon of low and high responders has also been identified in rats (Han, 1997). It is possible that different types of reactors might have different intensities and time-course of response in changes of $\alpha$ motoneuron excitability consequent to acupuncture. To clarify the picture, future studies would need to take this into account in their experimental design and analysis. 


\subsubsection{Change in $\alpha$-motoneuron excitability did not arise from inactivity}

Although the present study found insignificant changes in $\alpha$-motoneuron excitability immediately on removal of the needles with both short duration and sustained acupuncture, there appeared to be a steady increase in inhibitory effect with time in both experimental groups. Sustained acupuncture appeared to be more effective with significant changes evident 35 minutes after insertion of needles, or 15 minutes after withdrawal of the needles. The significant inhibition of $\alpha$-motoneuron excitability was not likely to be a result of the subjects lying inactive for 35 minutes. This was because no significant effect occurred in the 8 subjects who were assessed 35 minutes after short duration acupuncture. Hence the different effects must be attributed to the different duration of the intervention rather than the period of inactivity. Also, Dawson et al. (1987) demonstrated no change in $\alpha$-motoneuron excitability even 1 hour after commencement of the experiment. Therefore, the change demonstrated in this study was unlikely to be the effect of prolonged inactivity. The significant inhibition shown after sustained acupuncture, however, could be the result of greater sensory input from the longer period of needle stimulation eliciting greater response from the central nervous system.

\subsubsection{Effect of short duration acupuncture may not be passive}

Although there was no statistically significant change from baseline, short duration acupuncture also appeared to have some inhibitory effect on $\alpha$-motoneuron excitability. Graphical representation of the data (Figs. $3.23 \&$ 3.25) demonstrated a progressive inhibition of $\alpha$-motoneuron excitability after both short duration and sustained acupuncture. Statistical analysis, however, did not reveal a significant difference between the effects of short duration and sustained acupuncture on $\alpha$-motoneuron 
excitability at 15 minutes after the interventions, when the inhibitory effect of sustained acupuncture was found to be significant. The statistical result could be explained by the small number of subjects and the wide variation in responses. Further studies with larger number of subjects will be needed to clarify whether short duration acupuncture can or cannot exert significant inhibitory effect on $\alpha$-motoneuron excitability.

\subsection{Comparison of effect of acupuncture and other interventions on}

\section{$\alpha$-motoneuron excitability}

The effects on $\alpha$-motoneuron excitability from other therapeutic measures have also been studied by using the H-reflex. These therapeutic measures, which are generally employed in the treatment of muscle spasm or increased muscle tone, included tendon pressure (Kukulka et al., 1986), massage (Morelli et al., 1991; Morelli et al., 1998), spinal manipulation (Dishman \& Bulbulian, 2001; Dishman et al., 2002), sacro-iliac joint manipulation (Murphy et al., 1995), cervical traction (Bradnam et al., 2000) and transcutaneous electrical nerve stimulation (TENS) (Chang et al., 2001; Hardy et al., 2002). Except for TENS, the effects of these interventions were generally inhibitory. While sacroiliac joint manipulation was shown to produce an effect up to 15 minutes after the intervention (Murphy et al., 1995), the effects from the other therapeutic measures were either transitory or of short duration. By way of comparison, acupuncture has no immediate effect on $\alpha$-motoneuron excitability. However, the effect of sustained acupuncture was significant at 15 and 30 minutes after withdrawal of needles, indicating a delayed effect which was not induced by the other therapeutic measures. The different time course of effects on $\alpha$-motoneuron excitability suggested the involvement of different neural pathways and mechanisms when the different forms of physical therapeutic measures are applied. 


\subsection{Limitations of the Study}

This study had limitations which were related to the H-reflex methodology and the cross-over design of the experiments.

\subsubsection{Limitations related to changes in peripheral conditions}

When using the H-reflex methodology, it is of paramount importance to minimize changes in peripheral conditions, so that changes in the H-reflex could be ascribed to central nervous system effects of the intervention. During the experiment, the following steps were taken to achieve this. Once the subject had been seated in a comfortable position, the same posture was kept throughout the experiment. The right leg was always used for both collection of H-reflex recordings and for acupuncture. The angles of the right knee and ankle were always held fixed by strapping the right leg to a leg support and resting the right foot onto a foot rest. Electrodes were fixed to the skin with adhesive tapes, and special additional attention was paid to support the already fixed cathode behind the right knee with a piece of rubber and elastic crepe bandage. The subjects were kept warm throughout the experiments.

\subsubsection{Procedures for screening peripheral stability}

After the experiment, data were screened for peripheral stability by assessing the consistency of the slopes of M-wave recruitment curves in all trials. Three procedures were involved, namely, the single intraclass correlation analysis, the $y$-intercept method (Kerr et al., 2002b), and visual inspection of the M-wave recruitment curves. ICC from the single intraclass correlation analysis was set at 0.95 as criteria for stability. The single intraclass correlation analysis was convenient to use as a screening procedure. When the ICC was less than 0.95 , inspection of graphical representation of grouped 
$\mathrm{M}_{\text {slp }}$ in the trials always confirmed presence of subjects with unstable $\mathrm{M}_{\text {slp }}$. On the other hand, when the ICC was above 0.95 , the graphical representation was generally quite homogeneous. The $y$-intercept method (Kerr et al., 2002b) was very effective in refining the selection of data for inclusion, but could be too sensitive or not sensitive enough in some instances. Visual inspection of the M-wave recruitment curves helped to identify curves which almost overlapped but would be excluded using the $y$-intercept method alone (Fig. 2.6). Visual inspection of the grouped M-slopes graphical representation also helped in identifying unstable $\mathrm{M}_{\text {slp }}$ (Fig. 3.17B). Therefore, combination of the three methods appeared to be a reasonable procedure for selection of data for final analysis.

\subsubsection{Compensation for minor changes in peripheral conditions}

Notwithstanding all the procedures taken before and after the experiments, there were still minor changes in individual M-slopes across the trials, possibly due to normal variation. $\mathrm{M}_{\mathrm{slp}}$ represents the rate of recruitment of the axons of $\alpha$-motoneurons which innervate the corresponding muscle fibres, and can reasonably be used as a reference property of motoneurons to evaluate the reflexive $H_{\text {slp }} . H_{\text {slp }} / M_{\text {slp }}$ was, therefore, also used as a parameter for assessing $\alpha$-motoneuron excitability as proposed by Funase et al. (1994).

The results from analysis using $\mathrm{H}_{\text {slp }}$ alone appeared to be in agreement with those from using $\mathrm{H}_{\mathrm{slp}} / \mathrm{M}_{\mathrm{slp}}$ in most instances. However, a significant discrepancy occurred in Trial 4 of sustained acupuncture, extended observation series. Here, percentage change in $\mathrm{H}_{\text {slp }}$ was insignificant, but analysis of data from the same subjects using percentage change in $\mathrm{H}_{\mathrm{slp}} / \mathrm{M}_{\text {slp }}$ as the parameter was significant (Refer section 3.8, Summary of 
Results). Since percentage changes in both $H_{\text {slp }}$ and $H_{\text {slp }} / M_{\text {slp }}$ were found to be significant in Trial 4 of the sustained acupuncture series, the same finding would be expected in the extended series as well. Bearing in mind the number of subjects in the extended series was smaller ( 8 subjects) than in the non-extended series (15 subjects), $\mathrm{H}_{\mathrm{slp}} / \mathrm{M}_{\text {slp }}$ was probably a more sensitive parameter for assessing $\alpha$-motoneuron excitability than $\mathrm{H}_{\text {slp }}$ alone. This was in agreement with the findings of Funase et al. (1994).

\subsubsection{Limitations of cross-over design}

Another limitation from the cross-over design of the study was carry-over effect (Polit \& Hungler, 1996). The carry-over effect from the first intervention could influence the effect from the second intervention. In this study the carry-over effect was diminished by a minimum 2-weeks washout period between the interventions. Most of the subjects actually had the interventions a few weeks apart. This provided an adequately long wash-out period to reduce the effect of the first intervention on the second, so carryover effect was not considered an influential factor in this study.

\subsection{Intensity of Acupuncture Sensation and $\alpha$-Motoneuron}

\section{Excitability}

The presence of acupuncture sensation (deqi) is considered to be a pre-requisite for effective acupuncture by traditional acupuncturists (Cheng, 1987; Helms, 1995). However, difference in the subjective perception of needling sensation can occur among individuals and at different points on the same individual ( $\mathrm{Li}$ et al., 1983). Despite this, Li et al (1983) reported a close correlation of needling sensation with needling effect. 
They found strong needling sensation was associated with marked elevation of pain threshold and depression of somatosensory evoked potentials. The present study, however, showed no significant correlation between the intensity of subjective acupuncture sensation (deqi) and the changes in $\alpha$-motoneuron excitability in short acupuncture. This concurs with the finding by Roth et al. (1997) that deqi sensation did not correlate with the rise in serum cortisol level after manual acupuncture.

Although moderate negative correlations (-0.495 in Trial 3 and -0.470 in Trial 4) were demonstrated with the percentage change in $\mathrm{H}_{\text {slp }} / \mathrm{M}_{\text {slp }}$ after sustained acupuncture, these were not significant $(p=0.061$ in Trial 3 and $p=0.077$ in Trial 4$)$. The results could not, therefore, support the long held belief that the efficacy of acupuncture is related to the presence of a strong subjective acupuncture sensation (deqi), at least in the response of $\alpha$-motoneuron excitability. It is possible that the relationship between acupuncture sensation (deqi) and body response, even if present, is not a strong one. Therefore, the correlation was not significant when response changes were small as in short acupuncture, but approached significance with greater response changes in sustained acupuncture. In this regard, it is interesting to note that slowly conducting muscle afferent units, which presumably are responsible for the transmission of acupuncture input, could be activated by mechanical stimuli at threshold intensity which was not perceived as painful by volunteers (Marchettini, Simone, Caputi, \& Ochoa, 1996). Further study will be needed for better definition of the correlation. 


\subsection{Possible Mechanisms of Acupuncture Effect on $\alpha$-motoneuron}

\section{Excitability}

From the results of the study it can be seen that short duration manual acupuncture had no significant effect on $\alpha$-motoneuron excitability. Sustained manual acupuncture for 20 minutes with intermittent manipulation of needles to obtain acupuncture sensation (deqi), on the other hand, had a more potent inhibitory effect on $\alpha$-motoneuron excitability. The effect appeared to be delayed, reaching a level of significance at 15 minutes after acupuncture (or 35 minutes from the beginning of acupuncture). Further inhibitory effect was recorded at 30 minutes after withdrawal of needles. The pattern is similar to that of acupuncture analgesia (Research Group of Acupuncture Anesthesia, 1973; Ulett et al., 1998). The exact mechanism of acupuncture causing inhibition of $\alpha-$ motoneuron excitability is not known from this study. Because of the time course of the events, it is postulated that the inhibitory effect, which has a slow onset and continues to progress after cessation of acupuncture, is mainly mediated by neuro-humoral factors (Ulett et al., 1998). The possible mechanisms involved are discussed under the following headings:

- Effect of acupuncture on the mental state of the subjects

- Effect of acupuncture on $\alpha$-motoneurons

\subsubsection{The effect of acupuncture on the mental state of the subjects}

It is known that H-reflex amplitude can be affected by the mental state and awareness of the subject. H-reflex amplitude increases during alertness and is related to the percentage of alpha rhythm in the ongoing EEG (Schieppati, 1987). Alpha rhythm is the major rhythm seen in normal relaxed adults. A decreasing alpha index is accompanied by decreasing H-reflex amplitudes (van Boxtel, 1976). Acupuncture is also known to 
have a non-specific relaxing and calming effect. Reports of a feeling of well-being or relaxation following an acupuncture treatment are not uncommon (Helms, 1995). Levy and Matsumoto (1975) recorded changes in EEG from beta to alpha rhythm after acupuncture-like electrical stimulation to the sciatic nerve of rabbits. However, $\mathrm{Fu}$ and Zhang (1980) recorded only slight but insignificant increase in alpha waves in patients under acupuncture anaesthesia. No significant EEG change from very short duration acupuncture was demonstrated in healthy volunteers by Rosted, et al. (2001). On balance it seemed unlikely that acupuncture could influence the mental state of the subjects sufficiently to lead to a change of the $\alpha$-motoneuron excitability in this study.

\subsubsection{The effect of acupuncture on $\alpha$-motoneurons excitability}

During fatigue from a sustained maximal voluntary contraction, mean motoneuron discharge rates declines (Bigland-Ritchie et al., 1986). The increased inhibition of homonymous or heteronymous motoneurons was ascribed to increased discharges of groups III (A $\delta$ ) and IV (C) muscle afferents during muscle fatigue (Hayward et al., 1988). The action was presumably mediated by inhibitory interneurons in lamina VII of the spinal cord (Cleland, Rymer, \& Edwards, 1982). Further, both motor cortical and spinal motoneuron excitability were shown to be suppressed by tonic muscle pain (Le Pera et al., 2001), which was also thought to be mediated by activation of group III (A $\delta$ ) and IV (C) muscle afferents. Since $\mathrm{A} \delta$ and $\mathrm{C}$ muscle afferents transmit acupuncture stimuli to the central nervous system, it is likely that acupuncture will also lead to inhibition of the motoneurons through activation of these inhibitory afferents.

The neural pathway for suppression of $\alpha$-motoneuron excitability induced by acupuncture, muscle fatigue or muscle pain has not yet been defined. However, it is 
possible that a common pathway operates in these conditions since the initiating impulses are all conveyed by $\mathrm{A} \delta$ and $\mathrm{C}$ afferent fibres. It is interesting to note that Le Pera et al.(2001) found the motor cortex excitability to be inhibited by tonic muscle pain during the peak-pain period after injection of hypertonic saline into the muscle. Spinal motoneuron excitability remained unchanged during the peak-pain period but became inhibited during the following recovery phase. Functional magnetic resonance imaging also demonstrated activation of the hypothalamus, insula and motor cortex when the acupuncture point GB34 (Yanglingquan) was stimulated (Chiu et al., 2001). While the $\alpha$-motoneurons in the spinal cord are mainly under the control of signals transmitted from the motor cortex via the corticospinal tract, they are also modulated by indirect motor signals which travel through multiple accessory pathways involving the limbic system, the basal ganglia, cerebellum and various nuclei of the brainstem (Kandel, Schwartz, \& Jessell, 2000; Rekling et al., 2000). Most of these areas have also been implicated in hypothetical models of mechanism of acupuncture based on animal and human experiments (Bowsher, 1998; Cho et al., 2001; Pomeranz, 2001). On this basis, it is highly likely that acupuncture could have exerted its inhibitory effect on the $\alpha$ motoneurons by modulating signals in the higher brain centres whose descending fibres synapse directly on $\alpha$-motoneurons or influence them indirectly through spinal interneurons.

The time course of the changes in $\alpha$-motoneuron excitability in this study showed a delayed and progressive inhibitory effect even after cessation of acupuncture. From this observation it is postulated that the modulating effect of acupuncture also involves a humoral mechanism such as the release of neuropeptides or endorphins in the spinal cord or higher centres in the brain stem and hypothalamus. Although it has been shown that naloxone did not attenuate exercise-induced H-reflex suppression, which was 
hypothesized to involve endogenous opioid regulation (Bulbulian, 2002), it could be that the dosage of naloxone used $(10 \mathrm{mg})$ was too low. Sandrini et al (1999) demonstrated a marked facilitation of the H-reflex amplitude in normal subjects, but not in paraplegics, by administration of a higher dose of naloxone $(1.66 \mathrm{mg} / \mathrm{Kg})$. This suggested the existence of tonic inhibitory modulation of the H-reflex in normal subjects. Acupuncture stimulation could have enhanced this tonic inhibition via release of endorphins from the hypothalamus and brainstem. Neurons from the brainstem project to and modulate excitability of the $\alpha$-motoneurons in the spinal cord (Holstege \& Kuypers, 1987). Activation of the brainstem motor inhibitory systems and inactivation of the brainstem motor facilitatory systems may underlie acupunctureinduced inhibition of $\alpha$-motoneuron excitability (Habaguchi, Takakusaki, Saitoh, Sugimoto, \& Sakamoto, 2002; Mileykovskiy, Kiyashchenko, Kodama, Lai, \& Siegel, 2000). Further study is required to assess the role which endorphins might play in the process.

\subsection{Clinical Significance}

It is important to note that this study only demonstrated the physiological effects of acupuncture on $\alpha$-motoneuron excitability in normal subjects. It may be postulated that acupuncture alters $\alpha$-motoneuron excitability which, in turn, may influence motor unit activation and hence muscle activity. One has to be cautious when applying the information obtained to clinical situations.

In this study, sustained acupuncture was shown to have an inhibitory effect on $\alpha$ motoneuron excitability in normal subjects. Therefore, conditions where there is a pathological increase in $\alpha$-motoneuron excitability may theoretically benefit from the 
administration of acupuncture. This is encountered in patients with spasticity related to strokes (Higashi et al., 2001). Since sustained acupuncture for 20 minutes is more effective than short acupuncture for 5 minutes, sustained acupuncture should be preferably applied. The patients should also be informed of the lapse of time between acupuncture and the onset of a significant effect, so that they can have the right expectation from the outset.

This study also showed only a moderate but insignificant correlation between the perceived intensity of acupuncture sensation (deqi) and the inhibitory effect on $\alpha$ motoneuron excitability. Therefore, when acupuncture is administered, it is not essential to attempt to obtain the strongest bearable acupuncture sensation (deqi) if a decrease in $\alpha$-motoneuron excitability is the goal of the treatment.

\subsection{Future Work}

The study has shown the H-reflex methodology to be a useful tool for assessing the effect of acupuncture. It has also lead to a number of unanswered questions. While inhibition of $\alpha$-motoneuron excitability by acupuncture has been demonstrated, the underlying mechanism is still uncertain. Naloxone, at an adequate dosage, can be used to assess the part that $\beta$-endorphins might have played in acupuncture induced inhibition of $\alpha$-motoneuron excitability. The technique of transcranial magnetic stimulation can be used to study the effect of acupuncture on motor cortex excitability which, in turn, might have influenced excitability of the $\alpha$-motoneuron pool.

Although short duration acupuncture in this study did not result in significant inhibition of $\alpha$-motoneuron excitability, ongoing diminution of the excitability was noted for 30 
minutes after the intervention. In normal acupuncture practice, usually between 4-8 acupuncture points are used. Therefore, future studies with the use of 4-8 acupuncture points to mimic clinical acupuncture would be more appropriate. With increased acupuncture input, it might be possible that short duration acupuncture could also produce significant inhibition of $\alpha$-motoneuron excitability. Also, in clinical practice, patients become mobile after completion of the acupuncture session. To be able to apply the results of the investigation to clinical situations, the subjects should also be allowed to mobilize after the acupuncture period. Stick on electrodes which are left in place can be used to ensure that there is no change in stimulating and recording conditions for the H-reflex.

This study only examined the effect of segmental acupuncture using distal acupuncture points. In clinical situation, both local and distal points are used. For example, in low back pain patients, local points are needled in the lower back together with the distal points used in this study. It will be useful clinically to know if a combination of local acupuncture points and distal points are more effective than just distal points or just local points alone.

Another interesting phenomenon in acupuncture is the accumulative effect of treatments. To obtain maximum benefits, patients are generally asked to have a course of acupuncture which consists of 8-10 sessions at certain intervals such as twice per week. It will be of benefit to know if the effect of acupuncture on $\alpha$-motoneuron excitability is progressive and/or accumulative with the number of treatments and to assess the optimal intervals between treatments. This can be tested by repeating the $\mathrm{H}$ reflex assessment after acupuncture at 1,2,3 or 7 days intervals in different subjects. 
As noted earlier, the response of the body to acupuncture may differ according to the baseline level of excitability of motoneurons in neurological versus normal patients. Further studies involving symptomatic patients would be important for practical reasons because acupuncture is mainly used for treatment of different diseases or illnesses. 


\section{Participant Information Sheet}

\section{You are invited to take part in the following study:}

\section{The Effect of Acupuncture on Alpha Motoneuron Excitability}

Acupuncture has been widely and effectively used in the clinical relief of pain and muscle spasm. Mechanisms of its action have been ascribed to changes in levels of chemicals in the brain, called neurotransmitters and possibly by reflex relaxation of the muscles from impulses travelling in nerves from the sites of needling to the central nervous system and back to the muscles.

The purpose of this study is to apply a newer, more sensitive technique to investigate if there are significant changes in the excitability of the nerve cells (alpha motoneuron) in the spinal cord controlling muscle contraction and relaxation, both during and after acupuncture in normal subjects.

Anyone who is in good health and not suffering from any pain in the lower back or the lower limbs can join the study. It is recommended that for the few hours prior to the study, the participant does not drink any caffeine-containing beverage or partake in any strenuous activities.

The effect of acupuncture on the alpha motoneuron excitability will be evaluated by changes in a reflex elicited in the calf muscle by painless electrical stimulation of a nerve behind the knee. The study consists of two sessions at least 2 weeks apart. In one study, the acupuncture needles will not be stimulated after correctly inserted. In the other study, the acupuncture needles will be stimulated every 5 minutes in order to maintain an acupuncture sensation around the needles.

Each session will last approximately 1-1.5 hour. It is preferable, but not absolutely essential, that the same participants can take part in both sessions because this will give a greater power to the study.

Depending on the individual, some people may notice some discomfort when the nerve is stimulated and when the muscle contracts. There is also a sensation associated with acupuncture. This is generally localized and varies from a dull ache to numbness or tension in the muscle around the needle. Occasionally, when the nerve endings in the skin or muscle are stimulated, there may be a pinprick sensation, or sharp pain, but this is usually transient.

You have the right to terminate your participation in the study at any time you want, especially if you do not feel comfortable with the experience. 
The benefits include a personal experience of research participation, a personal experience of how acupuncture is like and how the H-reflex is being performed and recorded.

The information gathered will be confidential and there will be no name on the record sheet. Participants will be identified by code only and all information will be stored on computer disk in a locked facility.

You will have up to 2 weeks to consider the invitation. If you intend to participate, please contact Alex Chan at 479-8015. You are welcome to ask further questions and have all your queries dealt with before you sign the consent form.

Any concerns regarding the nature of this project should be notified in the first instance to the Project Supervisor. Concerns regarding the conduct of the research should be notified to the Executive Secretary, AUTEC, Madeline Banda, madeline.banda@aut.ac.nz, 9179999 ext. 8044.

\section{Supervisors of project:}

Principal Supervisor: Dr. Andrea Vujnovich, HOD, School of Physiotherapy, Faculty of Health Science, AUT. Ph: 307-9999, ext. 7812

Second Supervisor: Lynley Bradnam, Senior Lecturer, School of Physiotherapy, Faculty of Health Science, AUT. Ph: 307-9999, ext. 7065

\section{Researcher:}

Dr. Alex Chan, Masters Programme Student, General Practitioner and Medical Acupuncturist, 512, East Coast Road, Mairangi Bay, Auckland. Ph: 479-8015

\section{Approved by the Auckland University of Technology Ethics Committee on} 29.10.2001

AUTEC Reference number 01/89 


\section{APPENDIX II}

\section{Consent to Participation in Research}

Title of Project: The Effect of Acupuncture on Alpha-

Motoneuron Excitability

Project Supervisor: Dr. Andrea Vujnovich

Researcher: Dr. Alex Chan

- I have read and understood the information provided about this research project.

- I have had an opportunity to ask questions and to have them answered.

- I understand that I may withdraw myself or any information that I have provided for this project at any time prior to completion of data collection, without being disadvantaged in any way. If I withdraw, I understand that all relevant data, or parts thereof, will be destroyed

- I agree to take part in this research.

Participant signature:

Participant name:

Date:

Project Supervisor Contact Details:

Dr. Andrea Vujnovich, Head, School of Physiotherapy,

Faculty of Health Studies.

Ph: 917-9999 extension 9685

Approved by the Auckland University of Technology Ethics Committee on 29/10/2001

AUTEC Reference number 01/89 


\section{An Invitation}

\section{to \\ Participate in an Exciting Study}

Aim of the study: To evaluate the effect of acupuncture on alpha motoneuron excitability

\section{Requirements:}

1. Being in good health

2. Currently not suffering from any conditions affecting the lower back or lower limbs

Place: Neurophysiology Laboratory, AUT

Time: Two 1-1.5 hours sessions of your time will be involved.

What is involved: Procedure will involve electrical stimulation of a nerve behind the knee and insertion of two acupuncture needles in the same leg.

Benefits and risks: The benefits include a personal experience of research participation, a personal experience of how acupuncture is like and how the H-reflex is being performed and recorded. There is no risk associated with the procedure, which will be explained to you more fully before you decide whether you would consent to participate.

If you are interested, please contact:

Dr. Alex Chan at 479-8015

General Practitioner \& Medical Acupuncturist, Mairangi Bay, Auckland.

Dr. Andrea Vujnovich, Principal Supervisor, at 917-9999, ext. 9685

Head of Department, School of Physiotherapy, AUT.

Lynley Bradnam, Second Supervisor, at 917-9999, ext. 7065

Senior Lecturer, School of Physiotherapy, AUT. 


\section{APPENDIX IV}

\section{SUBJECT DATA RECORDING SHEET (1)}

Subject information:

\begin{tabular}{|l|l|}
\hline Subject code & \\
\hline DOB & \\
\hline Sex & \\
\hline Age & \\
\hline Any symptomatic lower back or lower limbs problems? & \\
\hline Previous experience with acupuncture? & \\
\hline Handedness & \\
\hline
\end{tabular}

Test information:

\begin{tabular}{|l|l|}
\hline Date of test & \\
\hline Time of test & \\
\hline Limb tested & \\
\hline Any previous coffee, etc. & \\
\hline
\end{tabular}

\begin{tabular}{|l|c|c|c|c|}
\hline & Initial & $5 \mathrm{~min}$. & $10 \mathrm{~min}$. & $15 \mathrm{~min}$. \\
\hline Intensity of deqi sensation & & & & \\
\hline
\end{tabular}

(Using a VAS on a horizontal line $10 \mathrm{~cm}$ long)

$\begin{array}{lllllllllll}0 & 1 & 2 & 3 & 4 & 5 & 6 & 7 & 8 & 9 & 10\end{array}$


SUBJECT DATA RECORDING SHEET (2)

Subject code:

Intervention:

Intervention I : Needle left in place after satisfactory insertion

Intervention II: Needle stimulated to maintain acupuncture sensation

H-reflex information:

\begin{tabular}{|c|c|c|}
\hline \multirow{2}{*}{ H-threshold (Hth) } & Intensity (mA) & \multirow[t]{2}{*}{ Amplitude (mV) } \\
\hline & & \\
\hline \multicolumn{3}{|l|}{ H-maximum (Hmax) } \\
\hline \multicolumn{3}{|l|}{ M-threshold (Mth) } \\
\hline \multicolumn{3}{|l|}{ M-maximum (Mmax) } \\
\hline Amplification & Limb tested & \\
\hline Pulse width & Intensity of C & or $\mathrm{X} 10$ \\
\hline
\end{tabular}

Recruitment Curve Increments:

\begin{tabular}{|c|c|c|c|}
\hline Test & $\mathrm{mA}$ & Test & $\mathrm{mA}$ \\
\hline 1 & $-25 \%$ & 22 & $+55 \%$ \\
\hline 2 & & 23 & \\
\hline 3 & & 24 & \\
\hline 4 & $-15 \%$ & 25 & $+85 \%$ \\
\hline 5 & & 26 & \\
\hline 6 & & 27 & \\
\hline $7 \quad$ (Hth) & & $28(\operatorname{Hmax})$ & \\
\hline 8 (Hth) & & 29(Hmax) & \\
\hline 9 (Hth) & & $30($ Hmax $)$ & \\
\hline 10 & $+15 \%$ & 31 & $+115 \%$ \\
\hline 11 & & 32 & \\
\hline 12 & & 33 & \\
\hline 13 & $+25 \%$ & 34 & $+150 \%$ \\
\hline 14 & & 35 & \\
\hline 15 & & 36 & \\
\hline 16 & $+35 \%$ & 37(Mmax) & \\
\hline 17 & & 38(Mmax) & \\
\hline 18 & & 39(Mmax) & \\
\hline 19 & $+45 \%$ & 40 & $+110 \%$ \\
\hline 20 & & 41 & \\
\hline 21 & & 42 & \\
\hline
\end{tabular}


APPENDIX V

Table of M-intercepts confidence intervals in short duration acupuncture study

\begin{tabular}{|c|c|c|c|c|c|c|c|}
\hline ID & Cl & M1slp & M2slp & M3slp & M4slp & M5slp & Comment \\
\hline \multirow[t]{2}{*}{ AV } & Upper & -112.00 & -107.36 & -124.65 & -116.12 & -107.63 & \multirow{2}{*}{ Data Accepted } \\
\hline & Lower & -82.24 & -88.19 & -90.70 & -100.22 & -103.5 & \\
\hline \multirow[t]{2}{*}{$A L$} & Upper & -166.82 & -158.16 & -159.36 & -158.26 & -148.83 & \multirow{2}{*}{ Data Accepted } \\
\hline & Lower & -138.83 & -128.04 & -141.02 & -134.27 & -129.1 & \\
\hline \multirow[t]{2}{*}{ CL } & Upper & -155.01 & -142.35 & -145.56 & -156.01 & & \multirow{2}{*}{$\begin{array}{l}\text { Data accept on } \\
\text { Special condition }\end{array}$} \\
\hline & Lower & -142.52 & -133.37 & -131.56 & -125.16 & & \\
\hline \multirow[t]{2}{*}{ DC } & Upper & -17.88 & -84.63 & -58.01 & -13.90 & & \multirow{2}{*}{ Data Excluded } \\
\hline & Lower & -3.99 & -53.63 & -29.16 & -2.20 & & \\
\hline \multirow[t]{2}{*}{ DR } & Upper & -224.04 & -197.59 & -243.76 & -141.99 & & \multirow{2}{*}{ Data Excluded } \\
\hline & Lower & -177.08 & -180.65 & -171.05 & -46.47 & & \\
\hline \multirow[t]{2}{*}{ ED } & Upper & -145.74 & -133.84 & -148.94 & -137.72 & & \multirow[t]{2}{*}{ Data Accepted } \\
\hline & Lower & -85.39 & -75.75 & -83.29 & -71.01 & & \\
\hline \multirow[t]{2}{*}{ EC } & Upper & -198.72 & -193.04 & -177.84 & -111.84 & -115.17 & \multirow{2}{*}{ Data Excluded } \\
\hline & Lower & -113.98 & -169.47 & -92.39 & 7.86 & 5.07 & \\
\hline \multirow[t]{2}{*}{ JK } & Upper & -218.61 & -217.64 & -229.66 & -220.68 & -234.02 & \multirow{2}{*}{ Data Accepted } \\
\hline & Lower & -191.69 & -185.58 & -190.35 & -201.10 & -190.30 & \\
\hline \multirow[t]{2}{*}{$\mathrm{JL}$} & Upper & -276.79 & -278.02 & -279.97 & -205.88 & & \multirow{2}{*}{ Data Excluded } \\
\hline & Lower & -259.78 & -186.09 & -250.61 & -128.79 & & \\
\hline \multirow[t]{2}{*}{ KV } & Upper & -195.76 & -193.52 & -195.51 & -202.85 & -88.79 & \multirow{2}{*}{$\begin{array}{l}\text { Date accepted } \\
\text { only up to M4sIp }\end{array}$} \\
\hline & Lower & -141.00 & -117.67 & -122.35 & -121.47 & -43.28 & \\
\hline \multirow[t]{2}{*}{ KM } & Upper & -85.88 & -77.12 & -89.65 & -74.54 & -80.26 & \multirow{2}{*}{ Data Accepted } \\
\hline & Lower & -27.82 & -18.96 & -27.07 & -17.68 & -26.10 & \\
\hline \multirow[t]{2}{*}{ LZ } & Upper & -125.29 & -120.30 & -125.42 & -122.70 & -127.33 & \multirow{2}{*}{ Data Accepted } \\
\hline & Lower & -65.48 & -79.51 & -54.08 & -56.24 & -100.89 & \\
\hline \multirow[t]{2}{*}{ MA } & Upper & -230.58 & -220.70 & -224.82 & -226.48 & & Acront \\
\hline & Lower & -105.31 & -127.75 & -66.24 & -68.11 & & a \\
\hline MM & Upper & -71.81 & -75.74 & -84.86 & -98.01 & & Data Acconted \\
\hline & Lower & -30.43 & -30.91 & -50.88 & -26.55 & & \\
\hline SM & Upper & -75.41 & -72.04 & -77.99 & -79.18 & -74.62 & Data Acronted \\
\hline & Lower & -55.03 & -54.88 & -59.09 & -59.71 & -54.45 & \\
\hline SK & Upper & -125.82 & -127.58 & -115.86 & -136.74 & & \\
\hline & Lower & -74.72 & -90.53 & -91.97 & -98.51 & & 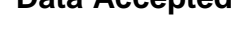 \\
\hline SW & Upper & -111.49 & -119.91 & -122.44 & -118.95 & & \\
\hline & Lower & -98.65 & -107.20 & -105.45 & -107.47 & & \\
\hline NS & Upper & -139.36 & -145.12 & -148.74 & -140.92 & -144.96 & Dato Acontod \\
\hline & Lower & -102.88 & -113.76 & -109.04 & -102.00 & -107.95 & \\
\hline TS & Upper & -171.64 & -172.23 & -167.08 & -186.47 & -181.51 & Data accepted on \\
\hline & Lower & -128.74 & -119.39 & -158.85 & -161.70 & -168.89 & Special condition \\
\hline TY & Upper & -170.47 & -159.89 & -170.99 & -179.64 & & Data accepted on \\
\hline & Lower & -138.37 & -132.46 & -121.79 & -161.49 & & \\
\hline
\end{tabular}


APPENDIX VI Table of M-intercepts confidence intervals in sustained acupuncture study

\begin{tabular}{|c|c|c|c|c|c|c|c|}
\hline ID & $\mathrm{Cl}$ & M1slp & M2slp & M3slp & M4sIp & M5slp & Comment \\
\hline AV & Upper & -102.92 & -105.26 & -107.92 & -108.00 & & \multirow{2}{*}{ Data Accepted } \\
\hline & Lower & -89.29 & -86.21 & -65.43 & -64.96 & & \\
\hline \multirow[t]{2}{*}{$A L$} & Upper & -90.31 & -78.89 & -69.01 & -94.43 & & \multirow{2}{*}{ Data Accepted } \\
\hline & Lower & -36.19 & -30.10 & -17.65 & -43.24 & & \\
\hline \multirow[t]{2}{*}{ CL } & Upper & -126.74 & -143.04 & -144.28 & -122.40 & & \multirow{2}{*}{ Data Accepted } \\
\hline & Lower & -97.72 & -112.01 & -111.40 & -90.01 & & \\
\hline \multirow[t]{2}{*}{$\mathrm{DC}$} & Upper & -74.38 & -67.22 & -68.40 & -67.96 & -74.37 & \multirow{2}{*}{ Data Accepted } \\
\hline & Lower & -45.75 & -15.57 & -25.31 & -53.73 & -64.80 & \\
\hline \multirow[t]{2}{*}{ DR } & Upper & -260.81 & -233.51 & -240.82 & -288.62 & & \multirow{2}{*}{ Data Excluded } \\
\hline & Lower & -216.28 & -199.70 & -138.44 & -253.10 & & \\
\hline \multirow[t]{2}{*}{ ED } & Upper & -144.24 & -152.88 & -173.83 & -144.35 & & \multirow{2}{*}{ Data Accepted } \\
\hline & Lower & -97.43 & -119.41 & -84.71 & -121.03 & & \\
\hline \multirow[t]{2}{*}{ EC } & Upper & -66.08 & -71.31 & -77.80 & -78.85 & -80.82 & \multirow{2}{*}{ Data Accepted } \\
\hline & Lower & -55.33 & -58.77 & -64.58 & -60.80 & -57.27 & \\
\hline \multirow[t]{2}{*}{$\mathrm{JK}$} & Upper & -288.44 & -288.36 & -299.56 & -301.25 & & \multirow{2}{*}{ Data Accepted } \\
\hline & Lower & -245.03 & -287.60 & -222.35 & -240.02 & & \\
\hline \multirow[t]{2}{*}{$\mathrm{JL}$} & Upper & -227.25 & -229.34 & -272.82 & -188.57 & & \multirow{2}{*}{ Data Excluded } \\
\hline & Lower & -140.50 & -147.89 & -212.34 & -134.38 & & \\
\hline \multirow[t]{2}{*}{ KV } & Upper & -152.83 & -160.80 & -150.92 & -149.86 & -143.68 & \multirow{2}{*}{ Data Accepted } \\
\hline & Lower & -136.63 & -132.02 & -140.29 & -137.92 & -127.53 & \\
\hline \multirow[t]{2}{*}{$\mathrm{KM}$} & Upper & -97.88 & -110.67 & -202.74 & -206.90 & -179.61 & \multirow{2}{*}{ Data Excluded } \\
\hline & Lower & -20.20 & -20.97 & -107.80 & -94.60 & -153.77 & \\
\hline \multirow[t]{2}{*}{ LZ } & Upper & -219.09 & -209.95 & -211.59 & -206.64 & -232.37 & \multirow{2}{*}{ Data Accepted } \\
\hline & Lower & -153.85 & -151.78 & -179.77 & -109.02 & -184.79 & \\
\hline \multirow[t]{2}{*}{ MA } & Upper & -136.97 & -129.81 & -142.59 & -140.74 & -107.26 & \multirow{2}{*}{ Data Accepted } \\
\hline & Lower & -86.5 & -84.95 & -85.11 & -85.64 & -98.34 & \\
\hline MM & Upper & -62.01 & -54.57 & -60.71 & -70.60 & & Data A conted \\
\hline & Lower & -15.49 & -0.16 & 8.88 & 2.10 & & \\
\hline SM & Upper & -140.04 & -125.63 & -76.54 & -76.91 & -84.51 & \\
\hline & Lower & -48.86 & -56.75 & -17.57 & -11.70 & -16.51 & \\
\hline SK & Upper & -158.83 & -168.95 & -254.07 & -195.42 & & Data Excluded \\
\hline & Lower & -114.79 & -135.68 & -212.53 & -142.35 & & \\
\hline SW & Upper & -136.51 & -135.16 & -137.09 & -134.64 & -129.37 & ccepted \\
\hline & Lower & -117.23 & -116.03 & -111.08 & -102.79 & -110.57 & \\
\hline NS & Upper & -66.06 & -67.08 & -79.95 & -79.26 & -77.95 & Data Accepted \\
\hline & Lower & -24.96 & -24.74 & -34.28 & -35.23 & -34.25 & \\
\hline TS & Upper & -109.91 & -154.76 & -162.27 & -161.56 & & Data Accepted \\
\hline & Lower & -30.06 & -63.10 & -74.69 & -48.68 & & \\
\hline TY & Upper & -178.93 & -178.08 & -177.14 & -173.47 & -167.83 & Data Accel \\
\hline & Lower & -125.15 & -121.56 & -97.91 & -103.61 & -96.13 & \\
\hline
\end{tabular}




\section{APPENDIX VII}

\section{Discarded experiments}

1. Unstable M-wave recruitment curve

Subjects: $\quad$ DC

DR $\times 2$
EC $\times 2$
JL $\times 2$
KM x 2
KV
SK

In these subjects the $95 \%$ confidence intervals for the $y$-intercept of the linear regression line fitted to the M-recruitment curves did not overlap (Appendix 5 and 6). The peripheral stimulating and recording conditions of their experiments were considered unstable and inconsistent. Therefore, data from these experiments were not included for final analysis.

Subject: $\quad$ LZ

In this subject, there was marked fluctuation in M-slopes across the trials (Fig. 3.17B). The peripheral stimulating and recording conditions in this experiment was also considered unstable and inconsistent. Therefore, data from this experiment were also excluded from final analysis.

2. Unstable H-reflex recruitment curve in control trials

Subject: $\quad$ MM

In this subject, $\mathrm{H}$-slopes in the control trials showed a marked change comparing to the rest of the subjects (Fig. 3.12A). Removal of data from this subject improved ICC of the group from 0.8621 to 0.9609 , which reflected stable baseline in the remaining 15 participants. 


\section{References:}

Acupuncture. (1997, Nov 3-5). NIH Consensus Statement Online.

Allison, S., \& Abraham, L. (1995). M-wave stability in H-reflex testing: analysis of three rejection criteria. Electromyogr clin Neurophysiol, 35, 165-168.

Alstermark, B., Kummel, H., Pinter, M. J., \& Tantisira, B. (1990). Integration in descending motor pathways controlling the forelimb in the cat. 17. Axonal projection and termination of $\mathrm{C} 3-\mathrm{C} 4$ propriospinal neurones in the $\mathrm{C} 6-\mathrm{Th} 1$ segments. Exp Brain Res, 81(3), 447-461.

Andersson, S., \& Lundeberg, T. (1995). Acupuncture--from empiricism to science: functional background to acupuncture effects in pain and disease. Med Hypotheses, 45(3), 271-281.

Baldry, P. (1998). Trigger point acupuncture. In J. Filshie \& A. White (Eds.), Medical Acupuncture (pp. 33-60). Edinburgh: Churchill Livingstone.

Bigland-Ritchie, B. R., Dawson, N. J., Johansson, R. S., \& Lippold, O. C. (1986). Reflex origin for the slowing of motoneuron firing rates in fatigue of human voluntary contractions. J Physiol, 379, 451-459.

Bing, Z., Villanueva, L., \& Le Bars, D. (1990). Acupuncture and diffuse noxious inhibitory controls: naloxone-reversible depression of activities of trigeminal convergent neurons. Neuroscience, 37(3), 809-818.

Bowsher, D. (1998). Mechanisms of acupuncture. In J. Filshie \& A. White (Eds.), Medical Acupuncture (pp. 69-82). Edinburgh: Churchill Livingstone.

Bradnam, L., Rochester, L., \& Vujnovich, A. (2000). Manual cervical traction reduces alpha-motoneuron excitability in normal subjects. Electromyogr clin Neurophysiol, 40(5), 259-266. 
Brown, A., \& Fyffe, R. (1981). Direct observations on the contacts made between Ia afferent fibres and alpha-motoneurons in the cat's lumbosacral spinal cord. $J$ Physiol (Lond), 313(1), 121-140.

Bucinskaite, V., Lundeberg, T., Stenfors, C., Ekblom, A., Dahlin, L., \& Theodorsson, E. (1994). Effects of electro-acupuncture and physical exercise on regional concentrations of neuropeptides in rat brain. Brain Res, 666(1), 128-132.

Bulbulian, R. (2002). Endogenous opioid effects on motoneuron pool excitability: Potential analgesic effect of acute exercise. J Manipulative Physiol Ther, 25(4), 209-215.

Bulbulian, R., \& Darabos, B. (1986). Motor neuron excitability: the Hoffmann reflex following exercise of low and high intensity. Med Sci Sports Exerc, 18(6), 697702.

Campbell, A. (1998). Methods of acupuncture. In J. Filshie \& A. White (Eds.), Medical Acupuncture (pp. 19-32). Edinburgh: Churchill Livingstone.

Campbell, A. (1999). The limbic system and emotion in relation to acupuncture. Acupuncture in Medicine, 17(2), 124-130.

Cao, X. (2002). Scientific bases of acupuncture analgesia. Acupunct Electrother Res, $27(1), 1-14$.

Casabona, A., Polizzi, M. C., \& Perciavalle, V. (1990). Differences in H-reflex between athletes trained for explosive contractions and non-trained subjects. Eur J Appl Physiol Occup Physiol, 61(1-2), 26-32.

Chang, Q. Y., Lin, J. G., \& Hsieh, C. L. (2001). Effect of manual acupuncture and transcutaneous electrical nerve stimulation on the H-reflex. Acupunct Electrother Res, 26(4), 239-251. 
Chapman, C. E., Sullivan.S.J., Pompura, J., \& Arsenault, A. B. (1991). Changes in hip position modulate soleus H-reflex excitability in man. Electromyogr. clin. Neurophyisol., 31, 131-143.

Chapman, C. R., Benedetti, C., Colpitts, Y. H., \& Gerlach, R. (1983). Naloxone fails to reverse pain thresholds elevated by acupuncture: acupuncture analgesia reconsidered. Pain, 16(1), 13-31.

Chen, X., Guo, S., Chang, C., \& Han, J. S. (1994). Optimal conditions for eliciting maximal electroacupuncture analgesia with dense-and-disperse mode stimulation. Am J Acupunct, 22(1), 47-53.

Chen, Y., Jen, Y., Teh, H., Yao, H., \& Shu, C. (1975). Studies on spinal ascending pathway for effect of acupuncture analgesia in rabbits. Scientia Sinica, 18, 651658.

Cheng, X. (Ed.). (1987). Chinese Acupuncture and Moxibustion (First Edition ed.). Beijing: Foreign Languages Press.

Chiang, C., Chang, C., Chu, H., \& Yang, L. (1973). Peripheral afferent pathway for acupuncture analgesia. Scientia Sinica, XVI(2), 210-217.

Chiu, J. H., Cheng, H. C., Tai, C. H., Hsieh, J. C., Yeh, T. C., Cheng, H., Lin, J. G., \& Ho, L. T. (2001). Electroacupuncture-induced neural activation detected by use of manganese-enhanced functional magnetic resonance imaging in rabbits. Am J Vet Res, 62(2), 178-182.

Cho, Z. H., Chung, S. C., Jones, J. P., Park, J. B., Park, H. J., Lee, H. J., Wong, E. K., \& Min, B. I. (1998). New findings of the correlation between acupoints and corresponding brain cortices using functional MRI. Proc Natl Acad Sci U S A, 95(5), 2670-2673.

Cho, Z. H., Wong, E. K., \& Fallon, J. H. (2001). Neuro-Acupuncture (Vol. 1). Los Angeles: Q-puncture, Inc. 
Chung, J. M., Fang, Z. R., Hori, Y., Lee, K. H., \& Willis, W. D. (1984). Prolonged inhibition of primate spinothalamic tract cells by peripheral nerve stimulation. Pain, 19(3), 259-275.

Chung, J. M., Lee, K. H., Hori, Y., Endo, K., \& Willis, W. D. (1984). Factors influencing peripheral nerve stimulation produced inhibition of primate spinothalamic tract cells. Pain, 19(3), 277-293.

Cleland, C. L., Rymer, W. Z., \& Edwards, F. R. (1982). Force-sensitive interneurons in the spinal cord of the cat. Science, 217(4560), 652-655.

Clement-Jones, V., McLoughlin, L., Tomlin, S., Besser, G. M., Rees, L. H., \& Wen, H. L. (1980). Increased beta-endorphin but not met-enkephalin levels in human cerebrospinal fluid after acupuncture for recurrent pain. Lancet, 2(8201), 946949.

Crayton, J. W., \& King, S. (1981). Inter-individual variability of the H-reflex in normal subjects. Electromyogr. clin. Neurophysiol., 21, 183-200.

Crone, C., Hultborn, H., Mazieres, L., Nielson, J., \& Pierrot-Deseilligny, E. (1990). Sensitivity of monosynaptic test reflex to facilitation and inhibition as a function of the test reflex size: a study in man and the cat. Exp. Brain Res., 81, 35-45.

Dai, J. L., Zhu, Y. H., Li, X. Y., Huang, D. K., \& Xu, S. F. (1992). C-fos expression during electroacupuncture analgesia in rats--an immunohistochemical study. Acupunct Electrother Res, 17(3), 165-176.

Danneels, L. A., Coorevits, P. L., Cools, A. M., Vanderstraeten, G. G., Cambier, D. C., Witvrouw, E. E., \& De, C. H. (2002). Differences in electromyographic activity in the multifidus muscle and the iliocostalis lumborum between healthy subjects and patients with sub-acute and chronic low back pain. Eur Spine J, 11(1), 1319. 
Davies, T. W., \& Lader, M. H. (1983). Effects of ankle joint angle on reflex and motor threshold intensities of soleus in man. J. Physiol., Lond., 339, 22P.

Davies, T. W., \& Lader, M. H. (1985). Determination of excitability in human proprioceptive reflexes: analysis and characteristics of EMB thresholds of postural muscle. Brain Res., 339, 19-26.

Dawson, N. J., Lippold, O. C., \& Milne, R. J. (1987). Contralateral intramuscular acupuncture-like electrical stimulation differentially changes the short-latency responses to muscle stretch. Exp Neurol, 98(1), 41-53.

Delwaide, P. (1977). Excitability of lower limb myotatic reflex arcs under the influence of caloric labyrinthine stimulation. Analysis of the postural effects in man. $J$ Neurol Neurosurg Psychiatry, 40(10), 970-974.

Dishman, J., \& Bulbulian, R. (2001). Comparison of effects of spinal manipulation and massage on motoneuron excitability. Electromyogr clin Neurophysiol, 41(2), 97106.

Dishman, J. D., Cunningham, B. M., \& Burke, J. (2002). Comparison of tibial nerve Hreflex excitability after cervical and lumbar spine manipulation. J Manipulative Physiol Ther, 25(5), 318-325.

Eke-Okoro, S. T. (1982). The H-reflex studied in the presence of alcohol, aspirin, caffeine, force and fatigue. Electromyogr clin Neurophysiol, 22, 579-589.

Enoka, R. (2002). Neuromechanics of human movement (3rd ed.). Champaign, IL: Human Kinetics.

Ernst, E., White, A. R., \& Wider, B. (2002). Acupuncture for back pain: meta-analysis of randomised controlled trials and an update with data from the most recent studies. Schmerz (Berlin, Germany), 16(2), 129-139. 
Farber, P. L., Tachibana, A., \& Campiglia, H. M. (1997). Increased pain threshold following electroacupuncture: analgesia is induced mainly in meridian acupuncture points. Acupunct \& Electrother Res, 22(2), 109-117.

Fisher, M. (1992). AAEM Minimonograph \#13: H reflexes and F waves: physiology and clinical indications. Muscle Nerve, 15, 1223-1233.

Fu, Y., \& Zhang, F. (1980). The changes of EEG under acupuncture anaesthesia (preliminary observations with EEG frequency analyzer), Advances in Acupuncture and Acupuncture Anaesthesia (pp. 319-320). Beijing: The People's Medical Publishing House.

Funase, K., Higashi, T., Yoshimura, T., Imanaka, K., \& Nishihira, Y. (1996). Evident difference in the excitability of the motoneuron pool between normal subjects and patients with spasticity assessed by a new method using H-reflex and Mresponse. Neurosci Lett, 203(2), 127-130.

Funase, K., Imanaka, K., \& Nishihira, Y. (1994). Excitability of the soleus motoneuron pool revealed by the developmental slope of the H-reflex as reflex gain. Electromyogr clin Neurophysiol, 34(8), 477-489.

Funase, K., \& Miles, T. (1999). Observations on the variability of the H reflex in human soleus. Muscle Nerve, 22(3), 341-346.

Goldberg, J., Seaborne, D. E., Sullivan, S. J., \& Leduc, B. E. (1994). The effect of therapeutic massage on H-reflex amplitude in persons with a spinal cord injury. Phys Ther, 74(8), 728-737.

Goldberg, J., Sullivan, S., \& Seaborne, D. (1992). The effect of two intensities of massage on H-reflex amplitude. Phys Ther, 72(6), 449-457.

Guo, Z., Zhou, M., Chen, X., \& Wang, R. (1997). Acupuncture methods for hemiplegic spasm. J Tradit Chin Med, 17(4), 284-288. 
Guyton, A., \& Hall, J. (2000). Textbook of medical physiology (10th ed.). Philadelphia, Pa. ; London: W. B. Saunders.

Habaguchi, T., Takakusaki, K., Saitoh, K., Sugimoto, J., \& Sakamoto, T. (2002).

Medullary reticulospinal tract mediating the generalized motor inhibition in cats:

II. Functional organization within the medullary reticular formation with respect to postsynaptic inhibition of forelimb and hindlimb motoneurons. Neuroscience, 113(1), 65-77.

Han, J. S. (1997). Physiology of acupuncture: review of thirty years of research. The Journal of Alternative and Complementary Medicine, 3, Supplement 1, S-101S108.

Han, J. S., Tang, J., Ren, M. F., Zhou, Z. F., Fan, S. G., \& Qiu, X. C. (1980). Central neurotransmitters and acupuncture analgesia. Am J Chin Med, 8(4), 331-348.

Hardy, S. G., Spaulding, T. B., Liu, H., Nick, T. G., Pearson, R. H., Hayes, A. V., \& Stokic, D. S. (2002). The effect of transcutaneous electrical stimulation on spinal motor neuron excitability in people without known neuromuscular diseases: the roles of stimulus intensity and location. Phys Ther, 82(4), 354-363.

Hashimoto, T., Akita, H., \& Aikawa, S. (1993). Analgesia induced by manual acupuncture: its potency and implication. Kitasato Arch Exp Med, 65 Suppl, 7382.

Hayward, L., Breitbach, D., \& Rymer, W. Z. (1988). Increased inhibitory effects on close synergists during muscle fatigue in the decerebrate cat. Brain Res, 440(1), 199-203.

Hayward, L., Wesselmann, U., \& Rymer, W. Z. (1991). Effects of muscle fatigue on mechanically sensitive afferents of slow conduction velocity in the cat triceps surae. J Neurophysiol, 65(2), 360-370. 
He, L. F., \& Dong, W. Q. (1983). Activity of opioid peptidergic system in acupuncture analgesia. Acupunct Electrother Res, 8(3-4), 257-266.

Helms, J. (1995). Acupuncture Energetics (First ed.). Berkeley, California.: Medical Acupuncture Publishers.

Henneman, E., Somjen, G., \& Carpenter, D. (1965a). Excitability and inhibitability of motoneurons of different sizes. J Neurophysiol, 28(3), 599-620.

Henneman, E., Somjen, G., \& Carpenter, D. O. (1965b). Functional significance of cell size in spinal motoneurons. J. Neurophysiol., 28, 560-580.

Higashi, T., Funase, K., Kusano, K., Tabira, T., Harada, N., Sakakibara, A., \& Yoshimura, T. (2001). Motoneuron pool excitability of hemiplegic patients: assessing recovery stages by using $\mathrm{H}-$ reflex and $\mathrm{M}$ response. Arch Phys Med Rehabil, 82(11), 1604-1610.

Hilgevoord, A. A. J., Koelman, J. H. T. M., Bour, L.J., \& Ongerboer de Visser, B. W. (1994). Normalisation of soleus H-reflex recruitment curves in controls and a population of spastic patients. Electroencephalogr clin Neurophysiol, 93, 202208.

Holstege, J. C., \& Kuypers, H. G. (1987). Brainstem projections to spinal motoneurons: an update. Neuroscience, 23(3), 809-821.

Homma, I., Endo, Y., \& Sakai, T. (1980). Inhibitory effect of acupuncture on the vibration-induced finger flexion reflex in man. Neurosci Lett, 19(2), 209-212.

Homma, S., \& Homma, I. (1982). Inhibitor effect of acupuncture on the vibrationinduced grasp reflex in man. Neurosci Lett, 32(2), 209-212.

Homma, S., Hori, Y., \& Yonezawa, T. (1985). The antagonistic effects of naloxone on acupuncture inhibition of the vibration-induced grasp reflex in man. Neurosci Lett, 61(1-2), 227-232. 
Homma, S., Nakajima, Y., \& Toma, S. (1985). Inhibitory effect of acupuncture on the vibration-induced finger flexion reflex in man. Electroencephalogr clin Neurophysiol, 61(2), 150-156.

Hopkins, J., Ingersoll, C., Edwards, J., \& Cordova, M. (2000). Changes in soleus motoneuron pool excitability after artificial knee joint effusion. Arch Phys Med Rehabil, 81(9), 1199-1203.

Hsieh, C. L. (2002). The physiological mechanisms of $2 \mathrm{~Hz}$ electroacupuncture: a study using blink and H reflex. Am J Chin Med, 30(2-3), 369-378.

Hsieh, J. C., Tu, C. H., Chen, F. P., Chen, M. C., Yeh, T. C., Cheng, H. C., Wu, Y. T., Liu, R. S., \& Ho, L. T. (2001). Activation of the hypothalamus characterizes the acupuncture stimulation at the analgesic point in human: a positron emission tomography study. Neurosci Lett, 307(2), 105-108.

Huang, C., Wang, Y., Han, J. S., \& Wan, Y. (2002). Characteristics of electroacupuncture-induced analgesia in mice: variation with strain, frequency, intensity and opioid involvement. Brain Res, 945(1), 20-25.

Hugon, M. (Ed.). (1973). Methodology of the Hoffmann reflex in man (Vol. 3). Basel: Karger.

Hui, K. K., Liu, J., Makris, N., Gollub, R. L., Chen, A. J., Moore, C. I., Kennedy, D. N., Rosen, B. R., \& Kwong, K. K. (2000). Acupuncture modulates the limbic system and subcortical gray structures of the human brain: evidence from fMRI studies in normal subjects. Hum Brain Mapp, 9(1), 13-25.

Irnich, D., Behrens, N., Gleditsch, J., Stor, W., Schreiber, M., Schops, P., Vickers, A., \& Beyer, A. (2002). Immediate effects of dry needling and acupuncture at distant points in chronic neck pain: results of a randomized, double-blind, shamcontrolled crossover trial. Pain, 99(1-2), 83. 
Ishikawa, K., Ott, K., Porter, R. W., \& al, e. (1966). Low frequency depression of H wave in normal and spinal man. Exp Neurol, 15, 140-156.

Jabre, J. (1981). Surface recording of the H-reflex of the flexor carpi radialis. Muscle Nerve, 4(5), 435-438.

Kandel, E. R., Schwartz, J. H., \& Jessell, T. M. (2000). Principles of Neural Science (4th ed.). New York: McGraw-Hill.

Karst, M., Rollnik, J. D., Fink, M., Reinhard, M., \& Piepenbrock, S. (2000). Pressure pain threshold and needle acupuncture in chronic tension-type headache - a double-blind placebo-controlled study. Pain, 88(2), 199-203.

Kawakita, K., \& Funakoshi, M. (1982). Suppression of the jaw-opening reflex by conditioning a-delta fiber stimulation and electroacupuncture in the rat. Exp Neurol, 78(2), 461-465.

Kawakita, K., \& Gotoh, K. (1996). Role of polymodal receptors in the acupuncturemediated endogenous pain inhibitory systems. Prog Brain Res, 113, 507-523.

Kerr, J. M., Vujnovich, A. L., \& Bradnam, L. (2002a). Changes in alpha-motoneuron excitability with positions that tension neural tissue. Electromyogr clin Neurophysiol, 42(8), 459-471.

Kerr, J. M., Vujnovich, A. L., \& Bradnam, L. (2002b). The intercept method: a novel method for establishing consistency of M-wave recruitment curves. Electromyogr clin Neurophysiol, 42(7), 423-432.

Kho, H., \& Robertson, E. (1997). The mechanisms of acupuncture analgesia: Review and Update. Am. J Acupunct., 25(4), 261-281.

Kniffki, K. D., Mense, S., \& Schmidt, R. F. (1978). Responses of group IV afferent units from skeletal muscle to stretch, contraction and chemical stimulation. Exp Brain Res, 31(4), 511-522. 
Kodachi, K., Sibuya, M., Endo, Y., \& Homma, I. (1987). Inhibitory effect of acupuncture on the finger flexion reflex induced by finger tip vibration. Neurosci Res, 4(5), 419-423.

Kohno, T., Kumamoto, E., Higashi, H., Shimoji, K., \& Yoshimura, M. (1999). Actions of opioids on excitatory and inhibitory transmission in substantia gelatinosa of adult rat spinal cord. $J$ Physiol, 518 ( Pt 3), 803-813.

Komiyama, T., Kawai, K., \& Fumoto, M. (1999). The excitability of a motoneuron pool assessed by the H-reflex method is correlated with the susceptibility of Ia terminals to repetitive discharges in humans. Brain Res, 826(2), 317-320.

Kong, J., Ma, L., Gollub, R. L., Wei, J., Yang, X., Li, D., Weng, X., Jia, F., Wang, C., Li, F., Li, R., \& Zhuang, D. (2002). A pilot study of functional magnetic resonance imaging of the brain during manual and electroacupuncture stimulation of acupuncture point (LI-4 Hegu) in normal subjects reveals differential brain activation between methods. J Altern Complement Med, 8(4), 411-419.

Kukulka, C. G., Beckman, S. M., Holte, J. B., \& Hoppenworth, P. K. (1986). Effects of intermittent tendon pressure on alpha motoneuron excitability. Phys Ther, 66(7), 1091-1094.

Lao, L., Bergman, S., Langenberg, P., Wong, R. H., \& Berman, B. (1995). Efficacy of Chinese acupuncture on postoperative oral surgery pain. Oral Surgery, Oral Medicine, Oral Pathology, Oral Radiology, and Endodontics, 79(4), 423-428.

Le Bars, D., Chitour, D., Kraus, E., Dickenson, A. H., \& Besson, J. M. (1981). Effect of naloxone upon diffuse noxious inhibitory controls (DNIC) in the rat. Brain Res, 204(2), 387-402. 
Le Bars, D., Dickenson, A. H., \& Besson, J. M. (1979). Diffuse noxious inhibitory controls (DNIC). I. Effects on dorsal horn convergent neurones in the rat. Pain, 6(3), 283-304.

Le Pera, D., Graven-Nielsen, T., Valeriani, M., Oliviero, A., Di Lazzaro, V., Tonali, P. A., \& Arendt-Nielsen, L. (2001). Inhibition of motor system excitability at cortical and spinal level by tonic muscle pain. Clin Neurophysiol, 112(9), 16331641.

Lee, J. H., \& Beitz, A. J. (1993). The distribution of brain-stem and spinal cord nuclei associated with different frequencies of electroacupuncture analgesia. Pain, 52(1), 11-28.

Leibing, E., Leonhardt, U., Koster, G., Goerlitz, A., Rosenfeldt, J. A., Hilgers, R., \& Ramadori, G. (2002). Acupuncture treatment of chronic low-back pain -- a randomized, blinded, placebo-controlled trial with 9-month follow-up. Pain, 96(1-2), 189-196.

Leong, R. J., \& Chernow, B. (1988). The effects of acupuncture on operative pain and the hormonal responses to stress. International Anesthesiology Clinics, 26(3), 213-217.

Leshnower, B. G., Potts, J. T., Garry, M. G., \& Mitchell, J. H. (2001). Reflex cardiovascular responses evoked by selective activation of skeletal muscle ergoreceptors. J Appl Physiol, 90(1), 308-316.

Levy, B., \& Matsumoto, T. (1975). Pathophysiology of acupuncture: nervous system transmission. Am Surg, 41(6), 378-384.

Li, S. C., Jiang, C. C., \& Chen, G. B. (1983). The relationship between needling sensation and acupuncture effects, with special reference to their ascending pathway in the spinal cord. Acupunct Electrother Res, 8(2), 105-110. 
Little, J., Hayward, L., \& Halar, E. (1989). Monopolar recording of H reflexes at various sites. Electromyogr clin Neurophysiol, 29(4), 213-219.

Liu, X., Zhu, B., \& Zhang, S. X. (1986). Relationship between electroacupuncture analgesia and descending pain inhibitory mechanism of nucleus raphe magnus.PG - 383-96. Pain, 24(3).

Lou, Z., Sun, W., Liu, Y., \& Tong, Z. (1992). A study of the regularity of pain threshold changes after trauma and the effect of electroacupuncture. Zhen Ci Yan Jiu, 17(3), 186-190.

Lundeberg, T., Eriksson, S., Lundeberg, S., \& Thomas, M. (1989). Acupuncture and sensory thresholds. The American Journal of Chinese Medicine, 17(3-4), 99110.

Magladery, J. W., \& McDougal, D. B. J. (1950). Electrophysiological studies of nerve and reflex activity in normal man: I. identification of certain reflexes in electromyogram and conduction velocity of peripheral nerve fibers. Bull Johns Hopkins Hosp, 86, 265-290.

Mann, F. (1992). Reinventing Acupuncture (First ed.). Oxford: Butterworth-Heinemann. Marchettini, P., Simone, D. A., Caputi, G., \& Ochoa, J. L. (1996). Pain from excitation of identified muscle nociceptors in humans. Brain Res, 740(1-2), 109-116.

Maryniak, O., \& Yaworski, R. (1987). H-reflex: optimum location of recording electrodes. Arch Phys Med Rehabil, 68(11), 798-802.

Mayer, D. J., Price, D. D., \& Rafii, A. (1977). Antagonism of acupuncture analgesia in man by the narcotic antagonist naloxone. Brain Res, 121(2), 368-372.

Mazzocchio, R., Scarfo, G., Mariottini, A., Muzii, V., \& Palma, L. (2001). Recruitment curve of the soleus H-reflex in chronic back pain and lumbosacral radiculopathy. BMC Musculoskelet Disord, 2(1), 4. 
Mense, S., \& Stahnke, M. (1983). Responses in muscle afferent fibres of slow conduction velocity to contractions and ischaemia in the cat. J Physiol, 342, 383-397.

Miglietta, O. (1970). Spinal motoneuron excitability in normal subjects and hemiplegic patients. Arch Phys Med Rehabil, 51(Dec 1970), 696-701.

Mileykovskiy, B. Y., Kiyashchenko, L. I., Kodama, T., Lai, Y.-Y., \& Siegel, J. M. (2000). Activation of Pontine and Medullary Motor Inhibitory Regions Reduces Discharge in Neurons Located in the Locus Coeruleus and the Anatomical Equivalent of the Midbrain Locomotor Region. J. Neurosci., 20(22), 8551-8558.

Milne, R. J., Dawson, N. J., Butler, M. J., \& Lippold, O. C. (1985). Intramuscular acupuncture-like electrical stimulation inhibits stretch reflexes in contralateral finger extensor muscles. Exp Neurol, 90(1), 96-107.

Morelli, M., Chapman, C. E., \& Sullivan, S. J. (1999). Do cutaneous receptors contribute to the changes in the amplitude of the H-reflex during massage? Electromyogr clin Neurophysiol, 39, 441-447.

Morelli, M., Seaborne, D., \& Sullivan, S. (1991). H-reflex modulation during manual muscle massage of human triceps surae. Arch Phys Med Rehabil, 72(11), 915919.

Morelli, M., Sullivan, S. J., \& Chapman, C. E. (1998). Inhibitory influence of soleus massage onto the medial gastrocnemius H-reflex. Electromyogr clin Neurophysiol, 38(2), 87-93.

Murphy, B., Dawson, N., \& Slack, J. (1995). Sacroiliac joint manipulation decreases the H-reflex. Electromyogr clin Neurophysiol, 35(2), 87-94.

Murray, J. B. (1995). Evidence for acupuncture's analgesic effectiveness and proposals for the physiological mechanisms involved. J Psychol, 129(4), 443-461. 
Okada, K., Oshima, M., \& Kawakita, K. (1996). Examination of the afferent fiber responsible for the suppression of jaw-opening reflex in heat, cold, and manual acupuncture stimulation in rats. Brain Res, 740(1-2), 201-207.

Ornung, G., Ottersen, O. P., Cullheim, S., \& Ulfhake, B. (1998). Distribution of glutamate-, glycine- and GABA-immunoreactive nerve terminals on dendrites in the cat spinal motor nucleus. Exp Brain Res, 118(4), 517-532.

Ostergaard, L., Fuglsang-Frederiksen, A., Sjo, O., Werdelin, L., \& Winkel, H. (1996). Quantitative EMG in cervical dystonia. Electromyogr clin Neurophysiol, 36(3), 179-185.

Pan, B., Castro-Lopes, J. M., \& Coimbra, A. (1996). Activation of anterior lobe corticotrophs by electroacupuncture or noxious stimulation in the anaesthetized rat, as shown by colocalization of Fos protein with ACTH and beta-endorphin and increased hormone release. Brain Res Bull, 40(3), 175-182.

Panizza, M. E., Nilsson, J., Roth, B. J., Basser, P. J., \& Hallett, M. (1992). Relevance of stimulation duration for activation of motor and sensory fibers: implications for the study of H-reflexes and magnetic stimulation. Electroencephalogr clin Neurophysiol, 85, 22-29.

Paquet, N., \& Hui-Chan, C. W. (1999). Human soleus H-reflex excitability is decreased by dynamic head-and-body tilts. J Vestib Res, 9(5), 379-383.

Park, H., Park, J., \& Lee, H. (2002). Does Deqi (needle sensation) exist? Am J Chin Med, 30(1), 45-50.

Pei, J., Sun, L., Chen, R., Zhu, T., Qian, Y., \& Yuan, D. (2001). The effect of electroacupuncture on motor function recovery in patients with acute cerebral infarction: a randomly controlled trial. J Tradit Chin Med, 21(4), 270-272. 
Pierrot-Deseilligny, E., \& Mazevet, D. (2000). The monosynaptic reflex: a tool to investigate motor control in humans. Interest and limits. Neurophysiol Clin, 30(2), 67-80.

Polit, D., \& Hungler, B. (1996). Research design for quantitative studies., Essentials of Nursing Research. Methods, Appraisals, and Utilization (4th ed., pp. 151-194). Philadelphia: Lippincott.

Pomeranz, B. (2001). Acupuncture Analgesia. In G. Stux \& R. Hammerschlag (Eds.), Clinical Acupuncture (pp. 1-28). Berlin: Springer.

Pomeranz, B., \& Paley, D. (1979). Electroacupuncture hypalgesia is mediated by afferent nerve impulses: an electrophysiological study in mice. Exp Neurol, 66(2), 398-402.

Ramnero, A., Hanson, U., \& Kihlgren, M. (2002). Acupuncture treatment during labour--a randomised controlled trial. BJOG: an International Journal of Obstetrics and Gynaecology, 109(6), 637-644.

Rekling, J. C., Funk, G. D., Bayliss, D. A., Dong, X.-W., \& Feldman, J. L. (2000). Synaptic Control of Motoneuronal Excitability. Physiol. Rev., 80(2), 767-852.

Research Group of Acupuncture Anesthesia, P. M. C. (1973). The role of some neurotransmitters of brain in finger-acupuncture analgesia. Sci Sin, 17, 112-130.

Reston, J. (1971) Now, let me tell you about my appendectomy in Peking... New York times, July 26, 1971.

Riddell, J. S., \& Hadian, M. (2000). Interneurons in pathways from group II muscle afferents in the lower-lumbar segments of the feline spinal cord. $J$ Physiol (Lond), 522(1), 109-123.

Rochester, L., Vujnovich, A., Newstead, D., \& Williams, M. (2001). The influence of eccentric contractions and stretch on alpha motoneuron excitability in normal 
subjects and subjects with spasticity. Electromyogr clin Neurophysiol, 41(3), $171-177$.

Romita, V. V., Suk, A., \& Henry, J. L. (1997). Parametric studies on electroacupuncture-like stimulation in a rat model: effects of intensity, frequency, and duration of stimulation on evoked antinociception. Brain Res Bull, 42(4), 289-296.

Rosted, P., Griffiths, P. A., Bacon, P., \& Gravill, N. (2001). Is there an effect of acupuncture on the resting EEG? Complement Ther Med, 9(2), 77-81.

Roth, L., Maret-Maric, A., Adler, R., \& Neuenschwander, B. (1997). Acupuncture points have subjective (needling sensation) and objective (serum cortisol increase) specificity. Retrieved 21.10.2002, 2002, from the World Wide Web: http://www.medical-acupuncture.co.uk/journal/may1997/one.shtml

Rudomin, P., Solodkin, M., \& Jimenez, I. (1987). Synaptic potentials of primary afferent fibers and motoneurons evoked by single intermediate nucleus interneurons in the cat spinal cord. J Neurophysiol, 57(5), 1288-1313.

Sabbahi, M., \& Khalil, M. (1990). Segmental H-reflex studies in upper and lower limbs of healthy subjects. Arch Phys Med Rehabil, 71(3), 216-222.

Sandrini, G., Milanov, I., Willer, J. C., Alfonsi, E., Moglia, A., \& Nappi, G. (1999). Different effect of high doses of naloxone on spinal reflexes in normal subjects and chronic paraplegic patients. Neuroscience Letters, 261(1-2), 5-8.

Schieppati, M. (1987). The Hoffmann reflex: a means of assessing the spinal reflex excitability and its descending control in man. Prog Neurobiol., 28, 345-376.

Shrout, P., \& Fleiss, J. (1979). Intraclass correlations: uses in assessing rater reliability. Psychological Bulletin, 86(2), 420-428. 
Sim, C.-K., Xu, P.-C., Pua, H.-L., Zhang, G., \& Lee, T.-L. (2002). Effects of electroacupuncture on intraoperative and postoperative analgesic requirement. Acupuncture in Medicine, 20(2-3), 56-65.

Sims, J. (1997). The mechansism of acupuncture analgesia: a review. Complementary Therapies in Medicine, 5, 102-111.

Sjolund, B., Terenius, L., \& Eriksson, M. (1977). Increased cerebrospinal fluid levels of endorphins after electro-acupuncture. Acta Physiol Scand, 100(3), 382-384.

Soper, W. Y., \& Melzack, R. (1982). Stimulation-produced analgesia: evidence for somatotopic organization in the midbrain. Brain Res, 251(2), 301-311.

Sullivan, S., Williams, L., Seaborne, D., \& Morelli, M. (1991). Effects of massage on alpha motoneuron excitability. Phys Ther, 71(8), 555-560.

Taborikova, H., \& Sax, D. S. (1969). Conditioning of H-reflexes by a preceding subthreshold H-reflex stimulus. Brain, 92, 203-212.

Takakura, N., Iijima, S., Kanamaru, A., Shibuya, M., Homma, I., \& Ohashi, M. (1996). Vibration-induced finger flexion reflex and inhibitory effect of acupuncture on this reflex in cervical spinal cord injury patients. Neurosci Res, 26(4), 391-394.

Takeshige, C., Oka, K., Mizuno, T., Hisamitsu, T., Luo, C. P., Kobori, M., Mera, H., \& Fang, T. Q. (1993). The acupuncture point and its connecting central pathway for producing acupuncture analgesia. Brain Res Bull, 30(1-2), 53-67.

Tanaka, T. H., Leisman, G., \& Nishijo, K. (1998). Dynamic electromyographic response following acupuncture: possible influence on synergistic coordination. Int J Neurosci, 95(1-2), 51-61.

Ulett, G. A., Han, S., \& Han, J. S. (1998). Electroacupuncture: mechanisms and clinical application. Biol Psychiatry, 44(2), 129-138. 
van Boxtel, A. (1976). The relation between monosynaptic spinal reflex amplitudes and some EEG alpha activity parameters. Electroencephalogr clin Neurophysiol, 40(3), 297-305.

Vujnovich, A., \& Dawson, N. (1994). The effect of therapeutic muscle stretch on neural processing. J Orthop Sports Phys Ther, 20(3), 145-153.

Wang, B., Tang, J., White, P. F., Naruse, R., Sloninsky, A., Kariger, R., Gold, J., \& Wender, R. H. (1997). Effect of the intensity of transcutaneous acupoint electrical stimulation on the postoperative analgesic requirement. Anesthesia and Analgesia, 85(2), 406-413.

Wang, K., \& Liu, J. (1989). Needling sensation receptor of an acupoint supplied by the median nerve--studies of their electro-physiological characteristics. Am J Chin Med, 17(3-4), 145-155.

Wang, K. M., Yao, S. M., Xian, Y. L., \& Hou, Z. L. (1985). A study on the receptive field of acupoints and the relationship between characteristics of needling sensation and groups of afferent fibres. Sci Sin [B], 28(9), 963-971.

Wang, Q. A., Mao, L. M., \& Han, J. S. (1990). The role of periaqueductal gray in mediation of analgesia produced by different frequencies electroacupuncture stimulation in rats. Int J Neurosci, 53(2-4), 167-172.

White, A. (1998). Electroacupuncture and acupuncture analgesia. In J. Filshie \& A. White (Eds.), Medical Acupuncture -- A Western Scientific Approach (pp. 153176). Edinburgh: Churchill Livingstone.

White, A. (1999). Neurophysiology of acupuncture analgesia. In E. Ernst \& A. White (Eds.), Acupuncture: a scientific appraisal. (pp. 60-92). Oxford: Butterworth Heinemann.

White, S., Fung, S., Jackson, D., \& Imel, K. (1996). Serotonin, norepinephrine, and associated neuropeptides: effects on somatic motoneuron excitability. In G. 
Holstege \& R. Bandler \& C. B. Saper (Eds.), The emotional motor system (pp. 183-199). Amsterdam: Elsevier.

Willer, J. C., Le Bars, D., \& De Broucker, T. (1990). Diffuse noxious inhibitory controls in man: involvement of an opioidergic link. Eur J Pharmacol, 182(2), $347-355$.

Williams, L., Sullivan, S., Seaborne, D., \& Morelli, M. (1992). Reliability of individual differences for H-reflex recordings. Electromyogr clin Neurophysiol, 32(1-2), $43-49$.

Williams, P., Bannister, L., Berry, M., Collins, P., Dyson, M., \& Dussek, J. (Eds.). (1995). Gray's Anatomy (38th ed.). New York: Churchill Livingstone.

Wong, A. M., Su, T. Y., Tang, F. T., Cheng, P. T., \& Liaw, M. Y. (1999). Clinical trial of electrical acupuncture on hemiplegic stroke patients. Am J Phys Med Rehabil, 78(2), 117-122.

Wu, S. (1996). 37 cases of myogenic torticollis treated by scalp and body acupuncture. $J$ Tradit Chin Med, 16(4), 278-280.

Wyon, Y., Hammar, M., Theodorsson, E., \& Lundeberg, T. (1998). Effects of physical activity and acupuncture on calcitonin gene-related peptide immunoreactivity in different parts of the rat brain and in cerebrospinal fluid, serum and urine. Acta Physiol Scand, 162(4), 517-522.

Yao, T. (1993). Acupuncture and somatic nerve stimulation: mechanism underlying effects on cardiovascular and renal activities. Scand J Rehabil Med Suppl, 29, 718.

Yu, Y., Wang, H., \& Wang, Z. (1995). The effect of acupuncture on spinal motor neuron excitability in stroke patients. Chung Hua I Hsueh Tsa Chih (Taipei), 56(4), 258-263. 